\title{
WORLD WIDE STUDY OF THE PERFORMANCE OF A SODIUM GUIDESTAR
}

\author{
Thesis \\ Submitted to \\ The School of Engineering of the \\ UNIVERSITY OF DAYTON \\ In Partial Fulfillment of the Requirements for \\ The Degree of \\ Master of Science in Electro-Optics
}

By

Steven M. Zuraski

UNIVERSITY OF DAYTON

Dayton, Ohio

May, 2014 


\section{WORLD WIDE STUDY OF THE PERFORMANCE OF A SODIUM GUIDESTAR}

Name: Zuraski, Steven Michael

APPROVED BY:

Steven T. Fiorino, Ph.D.

Advisory Committee Chairman
Mikhail Vorontsov, Ph.D.

Committee member

Professor - Electro-Optics Program

Department of Electrical Engineering

Department of Engineering Physics

Air Force Institute of Technology

Qiwen Zhan, Ph.D.

Committee Member

Professor - Electro-Optics Program

Department of Electrical Engineering

John G. Weber, Ph.D.

Associate Dean

School of Engineering
Tony E. Saliba, Ph.D.

Dean, School of Engineering

$\&$ Wilke Distinguished Professor 
(c) Copyright by

Steven Michael Zuraski

All rights reserved

2014 


\section{ABSTRACT \\ WORLD WIDE STUDY OF THE PERFORMANCE OF A SODIUM GUIDESTAR}

Name: Zuraski, Steven Michael

University of Dayton

Advisor: Dr. Steven Fiorino

This study quantifies the feasibility for a mobile sodium guidestar system. Simulations are run using the High Energy Laser End-to-End Operational Simulation (HELEEOS) software package with global sodium layer climatology data. Sodium layer data used was collected from the Optical Spectrograph and Infrared Imaging System (OSIRIS) sensor package on board the Odin satellite from 2005 through 2011 and provides a detailed global representation of the variable sodium layer occurring at an altitude of approximately $90 \mathrm{~km}$ in the atmosphere. This data is used in conjunction with the HELEEOS atmospheric propagation modeling to create realistic sodium guidestar models. The atmospheric effects for the laser propagation scattering model and creation of the sodium guidestar are defined in the worldwide probabilistic climatic database available in the HELEEOS software package. The simulations run evaluated the performance of a guidestar as viewed from along the propagation path and from nonpropagation path viewing angles for engagement scenarios in various locations on earth.

HELEEOS includes a fast-calculating, first principles, worldwide surface to $100 \mathrm{~km}$, with extensions above $100 \mathrm{~km}$ to account for the sodium layer, atmospheric propagation and characterization package. This package enables the creation of profiles of temperature, 
pressure, water vapor content, optical turbulence, atmospheric particulates and hydrometeors as they relate to line-by-line layer transmission, path and background radiance at wavelengths from the ultraviolet to radio frequencies. HELEEOS is able to produce realistic evaluations of laser propagation, imaging, and adaptive optics systems by use of an end to end directed energy propagation model that incorporates probabilistic, climatological data from temporally and spatially variable meteorological, aerosol, and turbulence profiles. Specifically, HELEEOS performs its propagation calculations utilizing the following algorithms, models and datasets: the Scaling for HEL and Relay Systems (SHaRE) scaling law algorithms, High Resolution Transmission (HITRAN) database for molecular absorption, Global Aerosol Dataset (GADS), Advanced Navy Aerosol Model (ANAM), the Adaptive Optical Compensation of Thermal Blooming (AOTB) model, various turbulence models, and other physics based atmospheric propagation algorithms. HELEEOS was developed by the United States Air Force Institute of Technology (AFIT) under the sponsorship of the High Energy Laser Joint Technology Office. 
Dedicated to my parents 


\section{ACKNOWLEDGEMENTS}

My special thanks are in order to Dr. Steven T. Fiorino, my advisor, for providing the time and software necessary for the work contained herein, and for directing this thesis and bringing it to its conclusion with patience and expertise.

I would also like to express my appreciation to everyone who has helped me with this

work. This includes Brannon Elmore, who provided updates to the software used for this thesis and Erin Dawkins, who facilitated the transfer of sodium layer data from the University of Leeds. 


\section{TABLE OF CONTENTS}

ABSTRACT




\section{LIST OF TABLES}

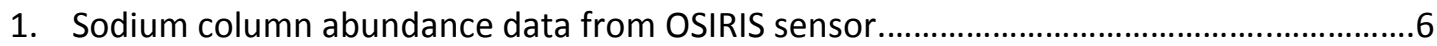

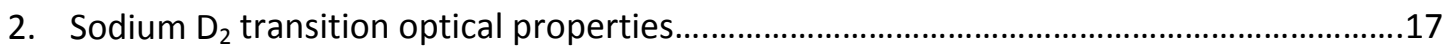

3. Parameters used for the sodium photon flux simulations................................................25

4. Sodium layer statistics for each month of collected data...................................................29 


\section{LIST OF ILLUSTRATIONS}

1. Sodium atom formation in the atmosphere.................................................................

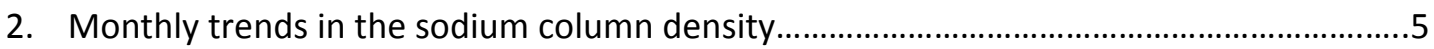

3. Column abundance of the sodium layer as a function of latitude and month....................7

4. The Keck laser guide star observatory using their sodium beacon ....................................10

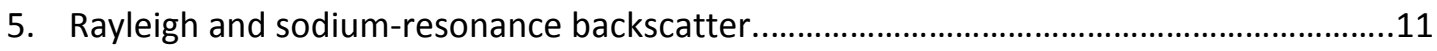

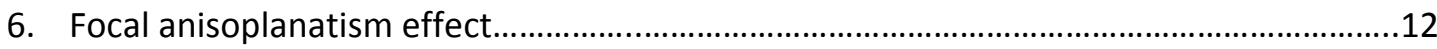

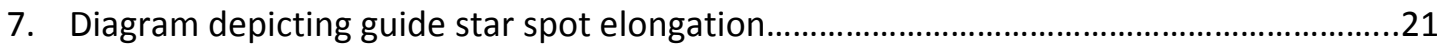

8. Sodium density profile used for double localized maximum example...............................22

9. Photon flux as seen by observer for the sodium profile in figure 7 above..........................23

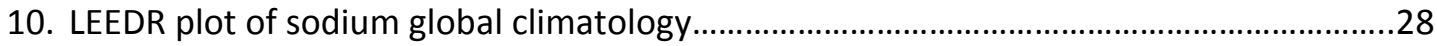

11. Monthly sodium layer mean density and standard deviation for the earth......................29

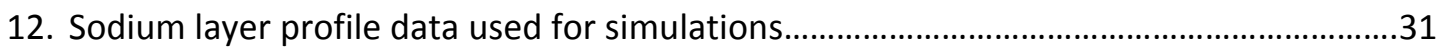

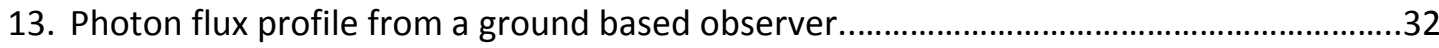

14. Photon flux profile from an air based observer...................................................................

15. Photon flux profile from a space based observer at a $100 \mathrm{~km}$ standoff.............................34

16. Photon flux profile from a space based observer at a $1000 \mathrm{~km}$ standoff...........................35

17. Photon flux profile from a space based side profile observe..............................................36

18-29. Sodium layer density vs altitude for all collected data across the globe........46-57

30-41. LEEDR plots of global sodium layer density for each month of the year..........58-64 
42-46. Maximum monthly sodium layer density for 35 locations on earth...............65-70 
LIST OF ABBREVIATIONS AND NOTATIONS

\begin{tabular}{ll} 
AFIT & Air Force Institute of Technology \\
ANAM & Advanced Navy Aerosol Model \\
AOTB & Adaptive Optics compensation for Thermal Blooming \\
DEW & Directed Energy Weapon \\
GADS & Global Aerosol Dataset \\
GOMOS & Global Ozone Monitoring by Occultation of Stars \\
HEL & High Energy Laser \\
HELEEOS & High Energy Laser End-to-End Operational Simulation \\
HITRAN & High Resolution Transmission \\
LEEDR & Laser Environment Definition and Reference \\
Na & Sodium atom abbreviation from periodic table \\
NAGS & Sodium Guidestar Simulator \\
NSLOT & Navy Surface Layer Optical Turbulence \\
OSIRIS & Optical Spectrograph and Infrared Imaging System \\
SHaRE & Scaling for HEL and Relay Engagement \\
\hline
\end{tabular}




\section{CHAPTER 1}

\section{INTRODUCTION}

Present in Earth's mesosphere from 80 to 105 kilometers there is a layer of concentrated sodium atoms. The existence of this layer is the result of approximately 30 tons of interplanetary dust that enters the Earth's atmosphere on a daily basis. The formation of this layer has been studied since the early 1970s, mostly from sodium resonant lidar systems. These lidar systems provide good special and temporal resolution of the sodium concentration profiles; however they are limited to the locations on earth where stations are. Satellite measurements are needed to provide a global picture of the behavior of the sodium layer. ${ }^{1,17}$, and 33

In recent years, a global view of the sodium layer has been studied using measurements from satellites. A limb scanning technique utilizing the sodium dayglow radiance at $589 \mathrm{~nm}$ is employed to capture sodium concentration measurements. For this study sodium layer data was provided by the University of Leeds. Data was collected globally over the span of multiple years utilizing a limb scanning technique performed by the Optical Spectrograph and Infrared Imaging System (OSIRIS) sensor on the Odin satellite. This data has been validated by simultaneous lidar collection. The sodium layer data was added to a Laser Environment Definition and Reference (LEEDR) database and a global sodium concentration profile was created. Results from this will be discussed in this paper. ${ }^{6}$ 
Incorporating the sodium layer concentration data added to LEEDR, makes it additionally available to the High Energy Laser End-to-End Operational Simulation (HELEEOS) toolset. New functionality was added LEEDR and HELEEOS so that a sodium guidestar can be simulated. The toolsets take into account atmospheric data and sodium layer data that are specific to a location on earth. This enables performance of a two way atmospheric propagation. At the sodium layer, a sodium wavelength laser interacts with the specific sodium layer profile data and calculations are performed to model the interaction of the sodium atoms with the photons from the laser. The toolsets will allow for photon flux calculations and a three dimensional analysis of a sodium guidestar. In this paper, three different scenarios are presented in a global environment to capture a sodium guidestar performance. The scenarios simulated are views of the sodium guidestar as viewed from a ground, air and space based sensor. How these calculations are performed and metrics on the results of the study are presented. $^{6 \text { and } 7}$ 


\section{CHAPTER 2}

\section{BACKGROUND}

\section{Sodium Layer Formation}

Meteoric ablation is the source of the layer of sodium atoms that exists globally from 80 to $105 \mathrm{~km}$ above earth's surface. The sodium layer was originally discovered in 1929 when radiation at $589 \mathrm{~nm}$ was observed in the night sky. This was later identified as the emissions being caused from a layer of sodium atoms that were concentrated in the upper mesosphere and lower thermosphere. It is estimated that approximately 30 tons of interplanetary dust enter and burn up in Earth's atmosphere every day, contributing to the formation of the sodium layer. ${ }^{1,12,} 23,27,29,31$, and 34

Several factors contribute to the high concentrations of sodium atoms between 80 and $100 \mathrm{~km}$ above earth's surface. In the atmosphere above approximately $80 \mathrm{~km}$ there is a sufficient concentration of atomic oxygen and hydrogen to recycle sodium compounds, such as sodium bicarbonate, back to atomic sodium. Below approximately $110 \mathrm{~km}$ the atmospheric density is high enough to convert $\mathrm{Na}+$ ions back to atomic sodium through cluster formation and dissociative electron recombination. This is why we see a high concentration of atomic sodium atoms from 80 to $110 \mathrm{~km}$ in Earth's atmosphere. In addition, current meteoric ablation models predict that relatively volatile metals such as sodium and potassium evaporate first upon entering Earth's atmosphere. This is predicted to occur around $93 \mathrm{~km}$ in the atmosphere. Out of the incoming extraterrestrial material, approximately $0.80 \%$ of the metal compounds are 
considered to be sodium, and when the sodium evaporates in the atmosphere about $92 \%$ will be atomic sodium atoms. Out of these, $50 \%$ will be ionized from hyper-thermal collisions with air molecules to become ionized. A diagram depicting this process described can be seen in Fig.

$1^{1,26,27,29,31,33 \text {, and } 34}$

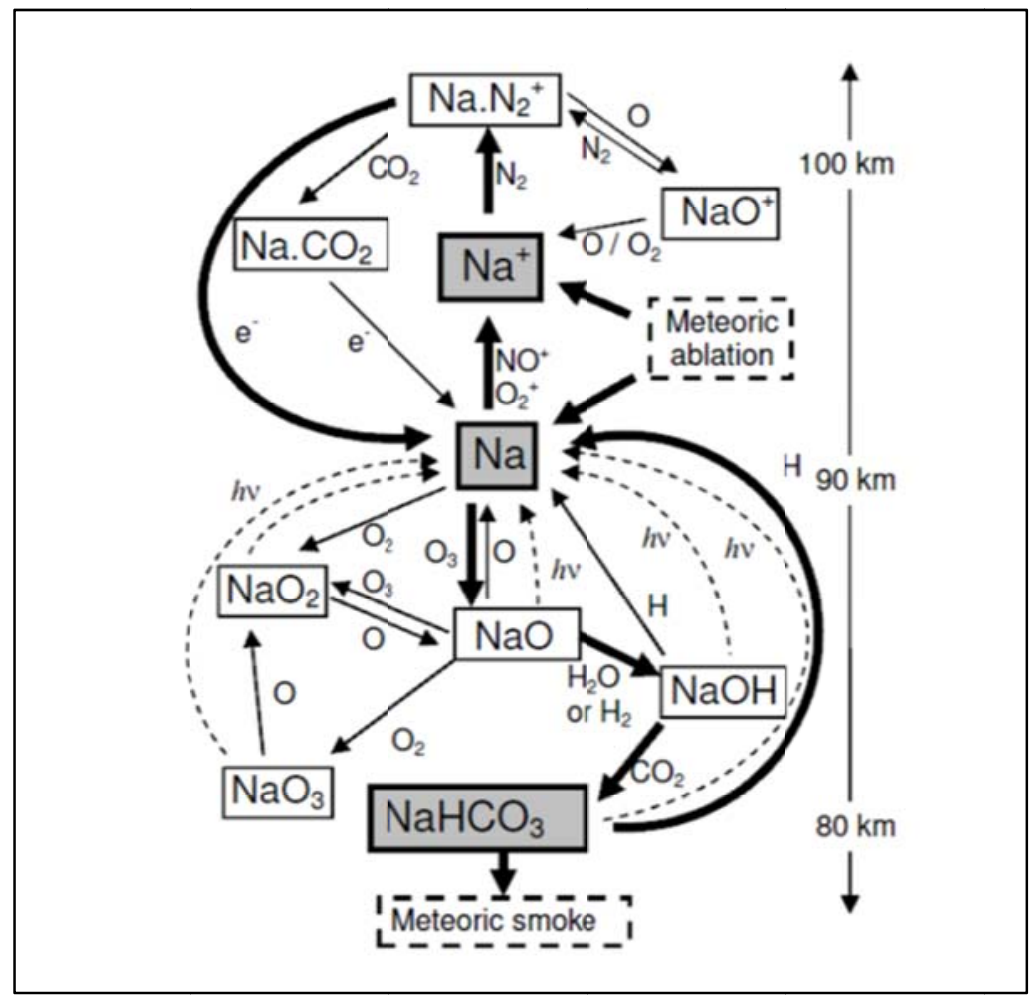

Figure 1: Graphical representation of sodium atom formation in the atmosphere ${ }^{3,29, \text { and } 34}$

\section{Traditional Sodium Layer Global Theory}

The sodium layer has been studied since the 1950s, first using ground based

photometry, then by resonant lidar systems, and most recently by limb-scanning spectroscopy from satellites. Data from the limb-scanning technique has produced a near global set of data that can be used as a reference for global trends in the sodium layer.

Traditional global sodium layer density theory, as presented by Ageorges and Dainty ${ }^{1}$, states that the sodium layer can vary by a full order of magnitude. At mid-latitudes, the sodium 
layer reaches its maximum in winter months, which is three times higher than the minimum in the summer months. In addition to the seasonal variation there is an erratic variation that can occur day to day or even in a time frame as short as ten minutes. These variations can be troublesome in designing a sodium guidestar system. The average sodium column density described by Ageorges is approximately 2 to $9 \times 10^{13} \mathrm{~m}^{-2}$, with peaks reaching as high as $4 \times 10^{9}$ $\mathrm{m}^{-2}$. This theory follows the trends as seen in the Fig 2. Data in Fig. 2 is from measurements from lidar systems, the OSIRIS sensor on the Odin satellite, and the Global Ozone Monitoring by Occultation of Stars (GOMOS) medium resolution spectrometer on the Envisat satellite. ${ }^{8,13,21 \text {, and }}$ 26

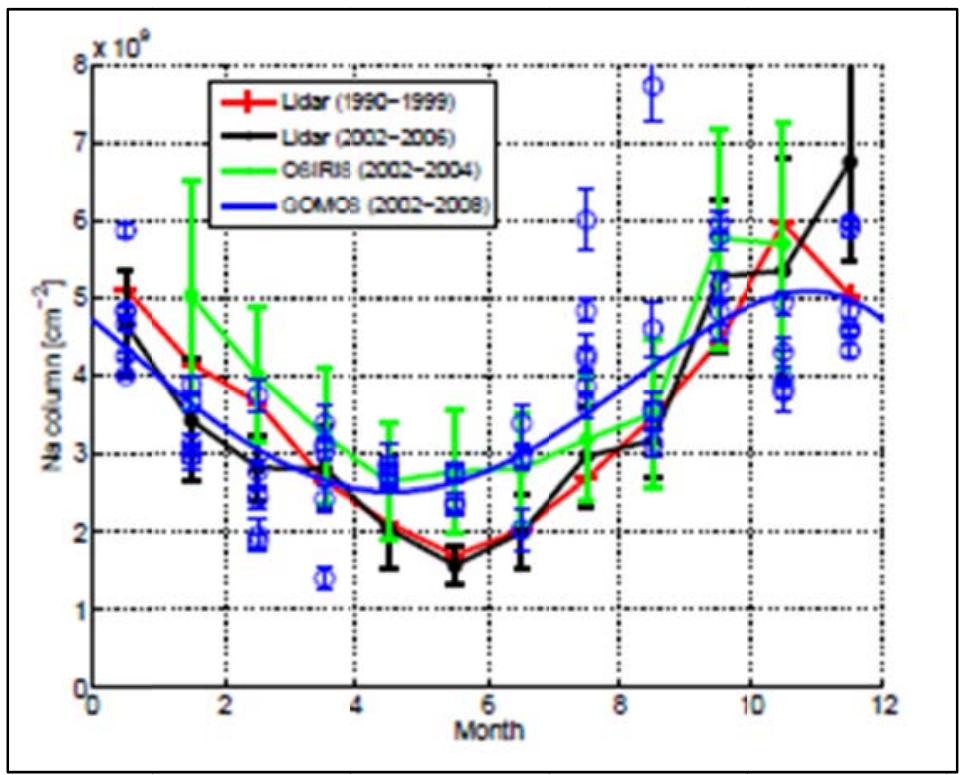

Figure 2: Monthly trends in the sodium column density at mid-latitudes as described by lidar systems in the 1990s, lidar systems in the early 2000s, and satellite measurements from the OSIRIS and GOMOS sensors ${ }^{11}$

In the mid to late 2000s Dr. Plane at the University of Leeds presented a new global reference for the sodium layer. His group based their global sodium layer reference off of measurements from the OSIRIS sensor from the Odin satellite. That data is the same data used in the LEEDR and HELEEOS toolsets presented in this paper. His group zonally averaged the 
collected data into 10 degree latitude bins on a monthly timescale from the first two years of collected data. Results from this averaging can be seen in the Table 1 and Fig. $3 .^{8 \text { and } 26}$

Table 1: Sodium column abundance data from OSIRIS sensor averaged into 10 degree latitude bins displayed per month. Units are in $10^{9}$ atoms $\mathrm{cm}-2$ and italicized values are extrapolated values for the contour plot below ${ }^{26}$

\begin{tabular}{|l|l|l|l|l|l|l|l|l|l|l|l|l|}
\hline Lat. & Jan & Feb & Mar & Apr & May & Jun & Jul & Aug & Sep & Oct & Nov & Dec \\
\hline 90 & 6.00 & 5.00 & 4.00 & 2.00 & 1.30 & 0.60 & 0.40 & 1.20 & 4.20 & 6.00 & 6.00 & 5.90 \\
\hline 80 & 5.40 & 5.00 & 4.63 & 2.35 & 1.72 & 0.64 & 0.33 & 1.59 & 4.24 & 6.00 & 5.90 & 5.80 \\
\hline 70 & 5.00 & 5.05 & 4.99 & 2.56 & 2.08 & 1.10 & 0.92 & 2.12 & 4.61 & 5.76 & 5.80 & 5.70 \\
\hline 60 & 5.90 & 5.86 & 5.06 & 2.83 & 2.21 & 1.45 & 1.58 & 2.59 & 4.29 & 5.82 & 5.80 & 5.70 \\
\hline 50 & 5.60 & 5.70 & 4.72 & 2.71 & 2.15 & 1.97 & 2.13 & 3.00 & 4.07 & 5.69 & 6.19 & 5.90 \\
\hline 40 & 5.12 & 4.78 & 3.94 & 3.20 & 2.66 & 2.86 & 2.94 & 3.25 & 3.44 & 5.80 & 5.80 & 5.04 \\
\hline 30 & 4.70 & 4.36 & 3.62 & 3.66 & 3.36 & 3.41 & 3.37 & 3.39 & 3.63 & 5.65 & 5.43 & 3.58 \\
\hline 20 & 4.50 & 4.09 & 2.90 & 3.87 & 3.75 & 3.45 & 3.43 & 3.14 & 3.55 & 4.90 & 4.59 & 3.66 \\
\hline 10 & 3.57 & 3.88 & 2.46 & 3.43 & 3.44 & 3.09 & 3.21 & 2.96 & 3.47 & 3.95 & 3.69 & 3.44 \\
\hline 0 & 3.58 & 3.70 & 1.89 & 2.33 & 2.94 & 2.87 & 3.02 & 2.84 & 3.03 & 3.50 & 3.71 & 3.48 \\
\hline-10 & 3.36 & 3.67 & 2.30 & 2.13 & 2.49 & 2.65 & 2.69 & 2.79 & 2.95 & 3.60 & 3.68 & 3.45 \\
\hline-20 & 3.26 & 3.74 & 2.48 & 2.19 & 5.80 & 5.75 & 5.85 & 5.40 & 5.00 & 4.85 & 3.70 & 3.45 \\
\hline-30 & 3.11 & 3.28 & 2.97 & 4.70 & 5.90 & 5.90 & 5.90 & 5.40 & 4.37 & 4.58 & 3.72 & 3.14 \\
\hline-40 & 2.82 & 3.52 & 3.09 & 5.00 & 6.00 & 6.00 & 6.00 & 5.50 & 4.64 & 4.02 & 3.45 & 2.61 \\
\hline-50 & 2.60 & 3.47 & 4.26 & 5.50 & 6.20 & 6.20 & 6.20 & 5.70 & 4.91 & 3.51 & 3.05 & 2.04 \\
\hline-60 & 1.93 & 2.46 & 4.66 & 5.70 & 6.40 & 6.50 & 6.40 & 5.80 & 4.80 & 3.38 & 2.52 & 1.50 \\
\hline-70 & 1.32 & 1.96 & 5.66 & 6.00 & 6.50 & 7.00 & 6.50 & 6.00 & 4.39 & 3.21 & 1.90 & 1.08 \\
\hline-80 & 0.93 & 1.35 & 3.00 & 6.00 & 6.50 & 7.30 & 6.50 & 6.20 & 4.50 & 3.18 & 1.70 & 0.80 \\
\hline-90 & 0.70 & 1.27 & 2.96 & 5.25 & 6.72 & 7.42 & 7.17 & 6.57 & 6.16 & 4.66 & 2.34 & 0.90 \\
\hline
\end{tabular}




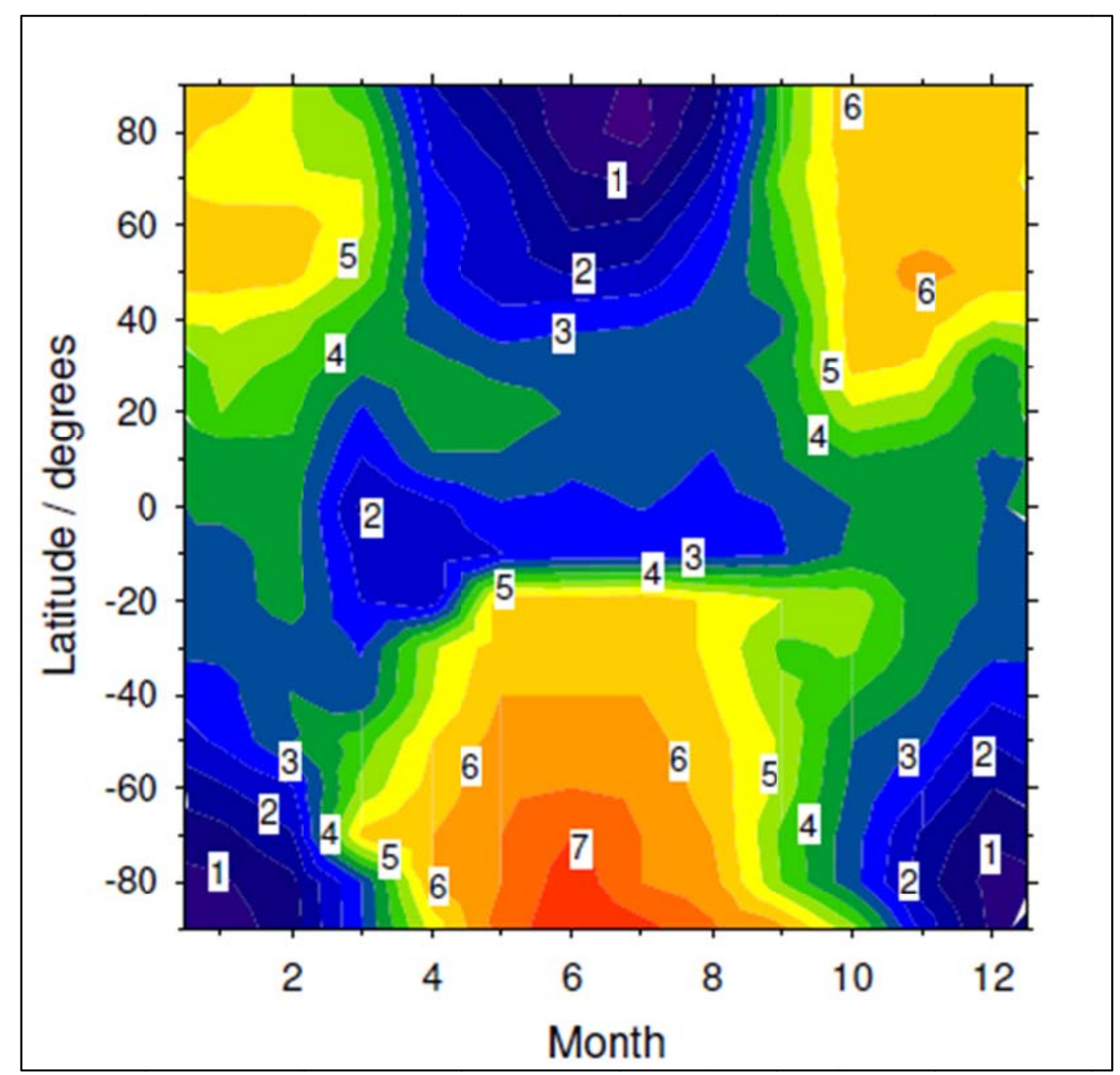

Figure 3: Column abundance of the sodium layer (units are $10^{9}$ atoms $\mathrm{cm}-2$ ) as a function of latitude and month ${ }^{26}$

\section{Collection Information from OSIRIS Sensor on Odin Satellite}

The most recently developed common form of satellite based measurement of the sodium layer employs limb-scanning measurements of the sodium resonance emission line in the dayglow. This is the technique that the OSIRIS sensor used on board the Odin satellite. The OSIRIS spectrograph is capable of collecting measurements from $280 \mathrm{~nm}$ to $800 \mathrm{~nm}$ with a resolution of $1 \mathrm{~nm}$. The sodium dayglow radiance profile is able to be measured in resolution section of approximately $2 \mathrm{~km}$ in altitude. This radiance profile is converted to sodium density 
profiles using optimal estimation theory and has also been validated against simultaneous lidar measurements that took place at Ft. Collins in Colorado. ${ }^{11,13,26, \text { and } 29}$

There are some limits to the collection capabilities of the Odin satellite and limbscanning technique in general. The Odin satellite is in a sun-synchronous orbit which causes some limitations on the collected data. This orbit allows for two measurements to be taken daily at approximately 0600 and 1800 local time. The collected data is also subject to times when the mesosphere is illuminated, meaning there is a solar zenith angle less than 92 degrees.

This results in no data being collected in high latitudes in winter months. ${ }^{8,26, \text { and } 28}$

It should be noted that the sodium density retrieval technique of limb-scanning the sodium resonant in the dayglow is subject to some uncertainty. From the OSIRIS sensor this uncertainty is plus or minus $10 \%$. This is comparable with modern lidar measurement techniques. $^{26}$

\section{Atmospheric Turbulence}

Small naturally occurring variations in temperature that are less than 1 degree Celsius cause random changes in the wind known as eddies. Motions in the atmosphere can also induce small temperature and humidity changes. These eddies are what scientists view as a turbulent atmosphere. Also, these small changes in temperature give rise to small variations in the atmospheric density, which influence the index of refraction. These small index of refraction variations can add cumulatively along the beam or view path and can cause significant inhomogeneity in the index of refraction profile, which will lead to changes in the course of a beam or blurred viewing. All this contributes to beam wondering, intensity fluctuations (scintillations), and beam spreading. 
The most common way to measure the strength of the turbulent atmosphere is described using the $\mathrm{C}_{\mathrm{n}}{ }^{2}$ term. This term is known as the refractive-index structure constant, but it is by no means a literal constant value. It varies with season, location, time, and altitude.

There are many models for the $C_{n}{ }^{2}$ term, with the most common being the Hufnagel-Valley $5 / 7$ boundary model. ${ }^{1 \text { and } 31}$

Laser Beacons

Laser beacons are needed to compensate for the turbulent atmosphere for application such as imaging objects in space. First we must consider using a natural guidestar to justify the reasoning for the need of a laser beacon. According to Tyson, there are approximately $1.45 \mathrm{e}^{0.96 \mathrm{mv}}$ stars per square radian in the sky. For a typical wave front sensor an $\mathrm{m}_{\mathrm{v}}=12$ or brighter star is needed. This results in roughly 150,000 stars per square radian. Thus for any specific region of the sky where a science object may be, there is likely not a magnitude 12 star within 10 micro-radians of the isoplanatic patch. In addition, some scientific objects of interest may be moving relatively quickly. Thus there is a need for artificial guide stars such as laser beacons. An example of what a laser guide star observatory looks like can be seen in the Fig. $4 .^{1}$ and 31 

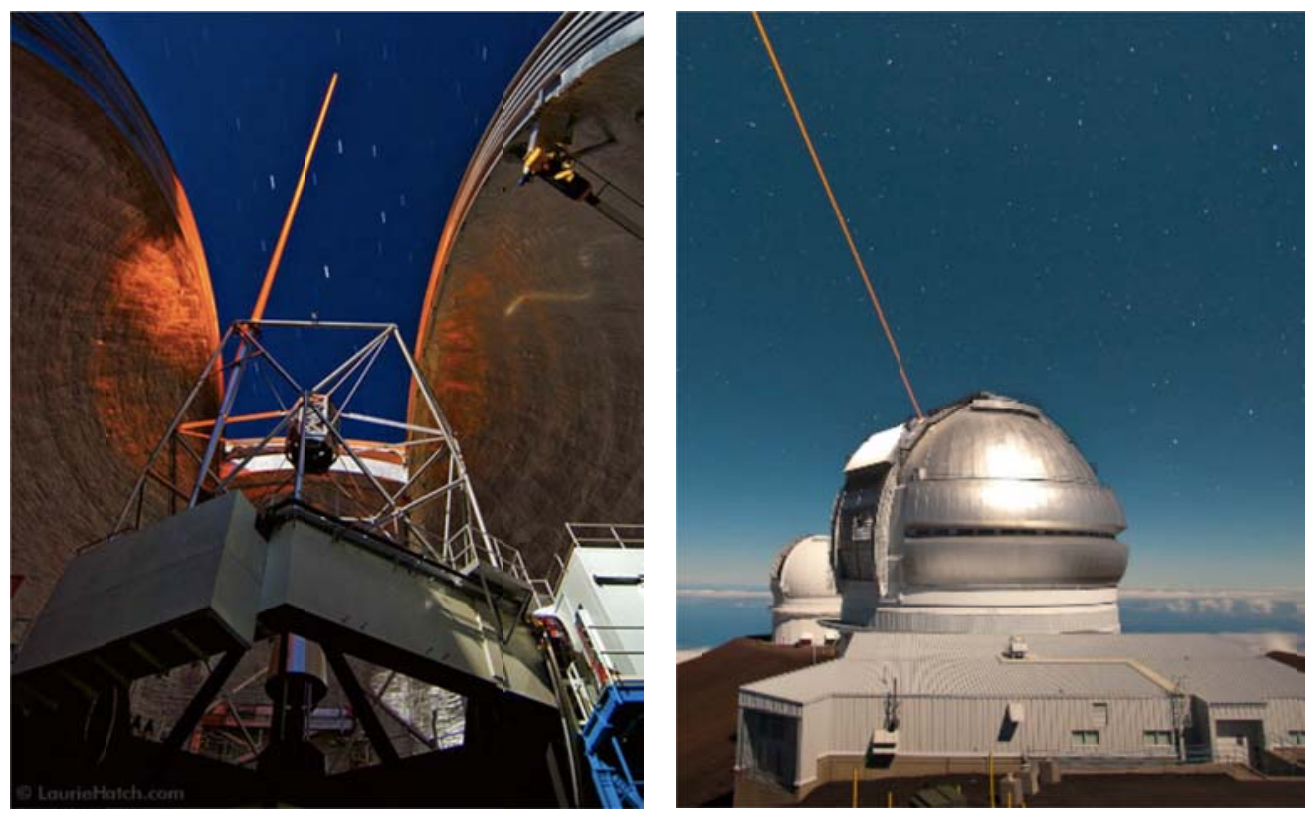

Figure 4: The Keck laser guide star observatory using their sodium beacon ${ }^{21}$

There are two basic types of laser beacons used for adaptive optics, a Rayleigh beacon and a sodium beacon. A representation of the backscatter vs altitude from a Rayleigh beacon and a Sodium-Resonance beacon can be seen Fig. 5. A sodium beacon is sometimes referred to as sodium resonance backscatter when the physical process that is actually taking place it the sodium atoms are absorbing the photon energy and then re-emitting the energy approximately 16 nanoseconds later. ${ }^{1,17,18, \text { and } 31}$ 


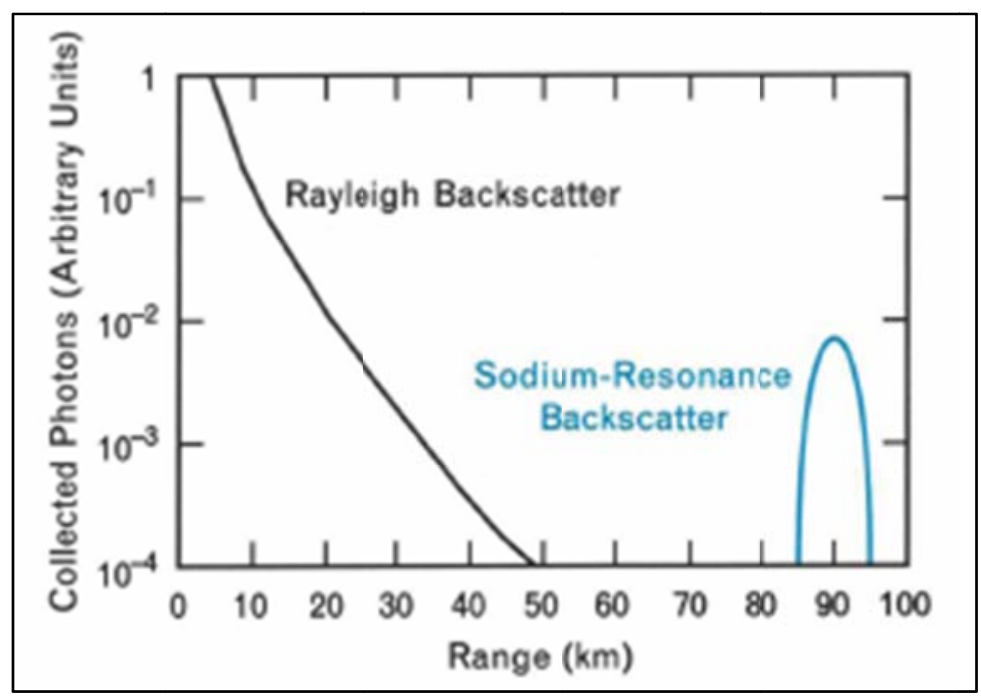

Figure 5: A relative representation of the backscatter collected from Rayleigh and sodiumresonance beacons ${ }^{17}$

Below $15 \mathrm{~km}$, it can be seen that a Rayleigh beacon will give a much higher backscattered signal. However, the Rayleigh beacon does not account for all the turbulent nature of the atmosphere. A sodium beacon is located at a high enough altitude that it accounts for nearly all the turbulent nature of the atmosphere. This is one of the reasons why the sodium layer receives attention in many adaptive optics studies. ${ }^{17 \text { and } 21}$

\section{Sodium Guidestar Systems and Focus Anisoplanatism}

A sodium guidestar system takes advantage of the naturally occurring sodium atoms at approximately $90 \mathrm{~km}$ in altitude in the atmosphere. A laser tuned to the $D_{2}$ transition at 589.2 $\mathrm{nm}$ is used to excite these sodium atoms. The sodium atom will absorb the laser lights and reemit the energy at nearly the same wavelength. This light that is re-emitted is what forms the artificial star in the sky used for adaptive optics systems. The sodium layer has a varying thickness that is about $10 \mathrm{~km}$ and varying density distribution profiles. This gives rise to elongated or uniquely shaped artificial stars when not viewed from along the beam path. ${ }^{19}$ 
One of the most important limiting factors when using a sodium guidestar system is focal anisoplanatism which is also known as the cone effect. This is due to the finite height of the sodium guidestar above the telescope being used. The traced rays from an artificial star form a cone in the cylinder being viewed, which leaves parts of the viewing that are turbulent and not accounted for. Also, any area above the artificial star is not sampled at all. This effect is less severe when using a sodium guidestar as compared to a Rayleigh guidestar due to the large difference in altitude above the observation telescope. Fig. 6 depicts focal anisoplanatism.

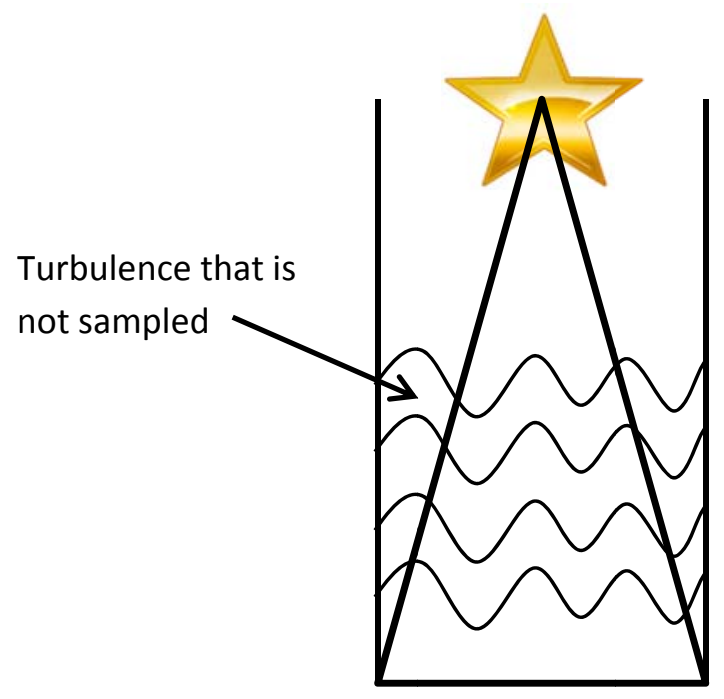

Figure 6: Focal anisoplanatism effect showing the unsampled turbulence in the beam path

Focal anisoplanatism sets the fundamental limitation to the quality of atmospheric correction that can be obtained with a sodium guidestar system. Mathematically focal anisoplanatism can be expressed as

$$
\sigma_{\phi}^{2}=\left(\frac{D}{d_{0}}\right)^{5 / 3}
$$


Where $\sigma_{\phi}^{2}$ is the phase variance for a telescope of diameter $D$. The value for $d_{0}$ is proportional to $\lambda^{6 / 5}$ and can be calculated for atmospheric turbulence profiles. The $d_{0}$ term is the aperture diameter over which the tilt error of a single beacon is $1 \mathrm{rad}^{2} .{ }^{1}$ and 31

\section{LEEDR and HELEEOS}

LEEDR was developed at AFIT's Center for Directed Energy and aids in the characterization of the atmosphere for a localized area on earth. LEEDR is a software package the allows for the creation of atmospheric profiles from the surface to $100 \mathrm{~km}$, with extensions above for the sodium layer, containing information on temperature, pressure, water vapor, optical turbulence, atmospheric particulates, hydrometeors as they relate to atmospheric transmission, path and background radiance at any wavelength band from ultraviolet to radio frequency, and sodium layer concentrations. An air-to-ground, air-to-air, or ground-to-space CFLOS calculator for 415 land sites worldwide is included in LEEDR. In general, LEEDR defines a well-mixed atmospheric boundary layer with a worldwide, probabilistic surface climatology based on season and time of day, and then computes the radiative transfer and propagation effects from the profile of meteorological variables. This allows LEEDR to accurately reconstruct atmospheric profiles that are representative of a specific location. ${ }^{6 \text { and } 7}$

HELEEOS was developed at AFIT's Center for Directed Energy and is a fast running Matlab-based scaling law model used for creating directed energy propagation simulations. Specifically, HELEEOS performs its propagation calculations utilizing the following algorithms, models and datasets: the Scaling for HEL and Relay Systems (SHaRE) scaling law algorithms, High Resolution Transmission (HITRAN) database for molecular absorption, Global Aerosol Dataset (GADS), Advanced Navy Aerosol Model (ANAM), the Adaptive Optical Compensation of Thermal Blooming (AOTB) model, various turbulence models, and other physics based atmospheric 
propagation algorithms. The objective of HELEEOS is to capture operational requirements, utilize a broad range of science and engineering analysis, assist in the evolutions of improved weapon system effectiveness through accurate engineering performance assessments, and establish a common understanding of directed energy possibilities. In order for HELEEOS to provide a realistic estimate of energy put on target it leverages two key components, LEEDR and correlated environmental effects on laser propagation. Some environmental effects include particulate absorption and scattering, optical turbulence, and CFLOS from virtually any location on the globe. HELEEOS supports a dynamic range of engagement scenarios which can contain a target, platform and up to two relays that can be configured in any three dimensional environment. ${ }^{6,7,23, \text { and } 32}$ 


\section{CHAPTER 3}

\section{METHODOLOGY}

\section{Sodium Data Ingestion into LEEDR}

Atmospheric sodium layer data was collected by the OSIRIS sensor on the Odin satellite from 2004 to 2011 using a limb-scanning technique in the Earth's dayglow. This data was processed into sodium density values vs altitude for specific collection locations on Earth. This process was performed and validated by the University of Leeds. The results of this, which consisted of eight years of sodium density profile data, are what were used to be ingested into the LEEDR toolset. In LEEDR each sodium layer data point consists of 16 measurements of the sodium column density that are at a resolution of $2 \mathrm{~km}$ ranging from $76 \mathrm{~km}$ to $106 \mathrm{~km}$ in altitude above the earth's surface. In total there are 57,637 data points specific to different location above earth's surface that were used. ${ }^{6 \text { and } 28}$

In LEEDR and HELEEOS these data points are used in two different ways. The first provides a view of the global climatology of the sodium layer maximums for each month. The data was separated by month and plotted as a visual magnitude representation on earth's surface. Results from this are discussed in the Results section and plots for each month can be seen in the Appendices I and II. The second way sodium density profile data points are used are to create a global selection of specific data to be used to set up a model that shows a sodium guide star system performance. When using LEEDR, there is a tab specific for use with the sodium layer. Here the user can select a location on earth and set up the engagement 
geometries. The tool will grab the nearest specific data point to be used and will run calculations to evaluate the sodium guidestar performance using that data. The strength of doing this in LEEDR is that other atmospheric effects specific to the location on earth can be used and real weather and atmospheric data can be ingested into the tool. This will give a real scenario model of how the system would perform in that region of the globe during a specific time. $^{6 \text { and } 7}$

\section{Atomic Sodium Physics}

The atomic sodium atom has a total of 11 electrons with a single valence electron outside the closed shells. The term symbol used for sodium at its ground state is $1 s^{2} 2 s^{2} 2 p^{6} 3 s^{2} S_{1 / 2}$ and the first excited state is $1 s^{2} 2 s^{2} 2 p^{6} 3 s^{2} P_{1 / 2,3 / 2}$. The interaction of the magnetic field moment of the electron with the magnetic field associated with the orbital motion of the electron leads to energy level splitting within the first excited state, with the two states being $3^{2} \mathrm{P}_{1 / 2}$ and $3^{2} \mathrm{P}_{3 / 2}$. These two states are separated in energy by approximately $520 \mathrm{GHz}$. The transition between the upper state $3^{2} \mathrm{P}_{3 / 2}$ and the ground state $3^{2} \mathrm{~S}_{1 / 2}$ are the cause for the sodium $\mathrm{D}_{2}$ absorption or emission at approximately $589 \mathrm{~nm} .{ }^{15,19}$, and 22

The sodium $D_{2}$ transition is chosen for the generation of sodium guide stars because it has a factor of 2 greater total line strength as compared to the $D_{1}$ transition. Properties of the sodium $D_{2}$ transition can be seen in the table below. ${ }^{15}$ and29 
Table 2: Sodium $D_{2}$ transition optical properties ${ }^{29}$

\begin{tabular}{|c|c|c|}
\hline \multicolumn{2}{|c|}{ Sodium $\mathrm{D}_{2}$ Transition Optical Properties } \\
\hline Wavelength (vacuum) & $\wedge$ & $589.158326 \mathrm{~nm}$ \\
\hline Wavelength (air) & $\lambda_{\text {air }}$ & $589.00035 \mathrm{~nm}$ \\
\hline Lifetime & $\tau$ & $16.249 \mathrm{~ns}$ \\
\hline $\begin{array}{c}\text { Saturation Intensity (isotropic } \\
\text { light polarization) }\end{array}$ & $\mathrm{I}_{\text {sat }}$ & $13.414 \mathrm{~mW} / \mathrm{cm}^{2}$ \\
\hline $\begin{array}{c}\text { Saturation Intensity ( } \pi \text {-polarized } \\
\text { light) }\end{array}$ & $\mathrm{I}_{\text {sat }}$ & $9.3901 \mathrm{~mW} / \mathrm{cm}^{2}$ \\
\hline $\begin{array}{c}\text { Saturation Intensity ( } \sigma \pm \text { polarized } \\
\text { light) }\end{array}$ & $\mathrm{I}_{\text {sat }}$ & $6.2600 \mathrm{~mW} / \mathrm{cm}^{2}$ \\
\hline $\begin{array}{c}\text { Resonant Cross Section (isotropic } \\
\text { light polarization) }\end{array}$ & $\sigma_{0}$ & $7.734 \times 10^{-8} \mathrm{~cm}^{2}$ \\
\hline $\begin{array}{c}\text { Resonant Cross Section ( } \pi- \\
\text { polarized light) }\end{array}$ & $\sigma_{0}$ & $1.105 \times 10^{-9} \mathrm{~cm}^{2}$ \\
\hline $\begin{array}{c}\text { Resonant Cross Section ( } \sigma \pm \\
\text { polarized light) }\end{array}$ & $\sigma_{0}$ & $1.657 \times 10^{-9} \mathrm{~cm}^{2}$ \\
\hline
\end{tabular}

In the case where we consider an isotropic pump field, the pumping field has equal components in all three possible polarizations, the sodium atom can be treated as a simple twolevel atom even though it has a fairly complicated hyperfine structure. Based on this we can describe the resonance fluorescence of the sodium atom. The steady state total photon scattering rate integrated over all directions and frequencies can be described by

$$
R_{s c}=\left(\frac{\Gamma}{2}\right) \frac{\left(I / I_{s a t}\right)}{1+4(\Delta / \Gamma)^{2}+\left(I / I_{s a t}\right)}
$$

Where $R_{s c}$ is the scattering rate, $\Gamma$ is the natural decay rate, $I$ is the incident intensity, $I_{\text {sat }}$ is the saturation intensity, and $\Delta$ is the detuning amount of the laser from the atomic resonance.

From this we can describe the saturation intensity and the corresponding on-resonance scattering optical cross section $\sigma$ which is

$$
\sigma_{0}=\frac{\hbar \omega \Gamma}{2 I_{s a t}}
$$


Where $\hbar$ is Plank's constant divided by $2 \pi$ and $\omega$ is the frequency. It should be noted that the saturation intensity is dependent on the polarization of the pumping light and thus the onresonance cross section as well. Values for $\mathrm{I}_{\text {sat }}$ and the corresponding optical cross sections can be seen in the table for the sodium $\mathrm{D}_{2}$ transition optical properties. For use in the LEEDR and HELEEOS tools only isotropic cases are considered. ${ }^{16,24, \text { and } 29}$

\section{Astronomical Brightness}

Astronomical brightness is defined by Tyson as the number of photons reaching the Earth's surface in a given unit of time. This term is dependent on the magnitude of the star in the visible pass band. The expression for astronomical brightness in units of photons $/ \mathrm{cm}^{2} \mathrm{~s}$ is

$$
B_{\text {astro }}=\left(4 \times 10^{6}\right) 10^{-m_{V} / 2.5}
$$

where $m_{v}$ is the visual magnitude of the observed star. To get an idea of the visual magnitude, the unaided human eye limit of vision in a dark location is equal to approximately an $m_{v}$ of 6 , and a sunlit geosynchronous satellite is roughly $m_{V}$ of $14 .{ }^{31}$

For a typical adaptive optic system utilizing an off the shelf Shack-Hartmann wave front sensor a guidestar of visual magnitude of approximately 12 is needed to achieve the wave front sensing requirement. This is found using the equation above and assuming a Fried's coherence length of $15 \mathrm{~cm}$, a wave front sensor operating at $100 \mathrm{~Hz}$, and where one subaperture is equal to one coherence length. ${ }^{1 \text { and } 31}$

On average there are 146029.44 natural stars of visual magnitude of 12 or brighter in the sky per square radian. Thus, any region of the sky where an object of scientific interest may be there is likely not a natural star, and therefor artificial stars like a sodium guidestar are needed. The equation that approximates the number of bright stars in the sky per square radian is $^{21}$ 


$$
\text { \# of Bright Stars }=1.45 e^{0.96 m_{V}}
$$

Using the photon flux calculation presented and the required brightness of a star needed for wave front correction we can get back to a rough sodium column density needed for an effective adaptive optic system. The actual sodium column density value required will vary slightly from system to system based on relevant system parameters. For our calculation we will assume typically used parameters to achieve an estimation of the sodium column density needed. Combine the photon flux equation and astronomical brightness equation and rearranging for the sodium column density we get

$$
\rho_{c o l}=\frac{F_{N a} 4 \pi z_{0}^{2}}{\eta T_{A}^{2} \sigma_{N a}} \frac{h c}{\lambda_{L G S} E}
$$

We require a visual magnitude equal to 12 , which corresponds to 40 photons per centimeter squared seconds. For this calculation we assume values of altitude equal to $92.5 \mathrm{~km}$, efficiency of $100 \%$ (ideal), a one way atmospheric transmission of $92 \%$, a $50 \mathrm{~W}$ continuous wave laser, and isotropic radiation from the sodium atoms. All other values are known constants. The choice of these values is representative of a laser system used for atmospheric correction using a sodium beacon. It is important to realize that choosing a more capable system will lower the required sodium column density. This results in a sodium column density of $8.8436 \times 10^{8}$ atoms per square centimeter. We use this number as a base line for the performance standard of a sodium guide star system being deployed in a global environment. ${ }^{1,2,9,10,11 \text {, and } 31}$

\section{Calculation of Photon Flux}

The photon flux calculation is performed based on methodology presented in common guidestar reference books such as those written by Ageorges and Dainty or by Tyson. This methodology is coupled with input parameters from LEEDR's atmospheric tools and databases that are specific to locations chosen on the earth. Combining these with specific sodium layer 
density profile data and laser input parameters, the photon flux from a sodium guidestar as viewed from an observer at any location relative to the radiating guidestar can be calculated. The photon flux calculation used is

$$
F_{\text {Sodium }}=\eta T_{A}^{2} \frac{\sigma_{N a} \rho_{c o l}}{4 \pi z_{0}^{2}} \frac{\lambda_{L G S} E}{h c}
$$

Where $F$ is the sodium guidestar return flux, $\eta$ is the sensor efficiency, $T_{A}$ is the atmospheric transmission, $\sigma$ is the sodium resonant backscatter cross section, $\rho$ is the sodium column density, $\lambda$ is the wavelength, $E$ is the energy of the laser, $z_{0}$ is the column height, $h$ is Plank's constant and $\mathrm{c}$ is the speed of light. This calculation is performed at each point in each of the screens and then summed as seen from the observer. The sodium column abundance and height are from the collected OSIRIS sensor data. The detector efficiency, laser energy, and wavelength (has to be resonance to perform calculation) are user inputs. The atmospheric transmission is calculated using LEEDR and HELEEOS using already existing toolsets for each point in the screens. The resonant cross section for sodium for isotropic radiation was chosen to be used. This cross section selection can be changed for accounting for polarization effects. What makes this calculation unique is that each point in the grid created is calculated separately and then summed together all based off of data that is specific to a location and time on earth. LEEDR and HELEEOS have the ability to ingest specific weather and atmospheric data to create a transmission profile unique to a location and time. This is combined with specific data points of the sodium layer density profile that closest match that specific data and time. Together this creates a realistic engagement model relative to a specific location and time on the globe. ${ }^{1,6,7}$, and 31

Spot Elongation 
Using the observer mode in LEEDR and HELEEOS allows for viewing of the spot elongation. A schematic depiction of the effects of spot elongation are shown in Fig 7.

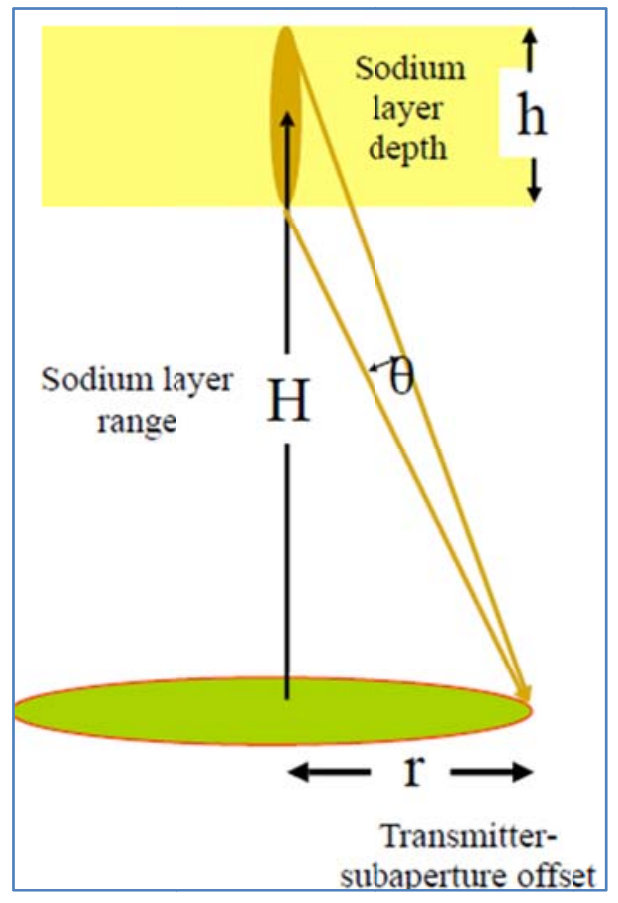

Figure 7: Diagram depicting how spot elongation occurs and can be viewed geometrically ${ }^{14}$

Now the elongation angle can be calculated by

$$
\theta=\tan ^{-1}\left(\frac{H+h / 2}{r}\right)-\tan ^{-1}\left(\frac{H-h / 2}{r}\right)
$$

In LEEDR and HELEEOS this effect can be seen while in off-axis observer mode. The geometries will be calculated and the photon flux screens from the guidestar will be added in a three dimensional volume and then compressed into a two dimensional space based on the observer location in regard to the guidestar. A calculated FOV of the observer needs to be used to fully capture the effects of the spot elongation. If a FOV is chosen that is too large, the resolution needed will likely not be available in the calculation to see the spot. If a FOV is chosen that is too narrow or small, then the whole sodium layer depth will not be captured and only a small subset of the guidestar will be seen. The angle, theta, should be calculated prior to using an off-axis observer in order to fully see the effects of spot elongation. ${ }^{23}$ 
The effects of a changing sodium layer density profile will also be seen in the spot elongation when vied from off axis. In the extreme case when the observer is directly perpendicular to the laser guide star, the influence of the sodium layer density profile can be seen. In some special cases a double localized maximum can be seen. An example of this can be seen in Fig. 8 and Fig. 9.

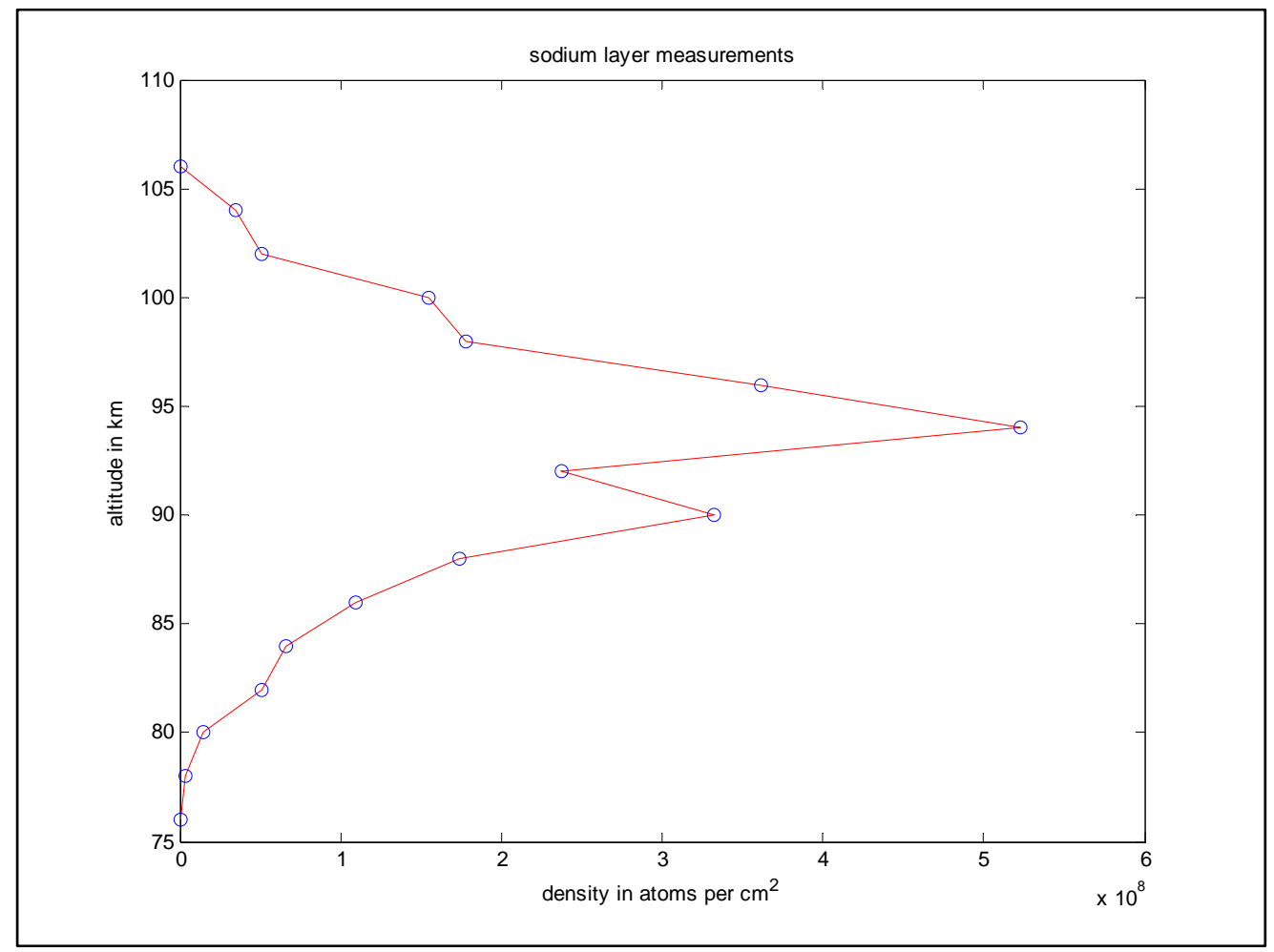

Figure 8: Sodium density profile used for double localized maximum example. This is actual measured sodium layer data from the OSIRIS sensor. 


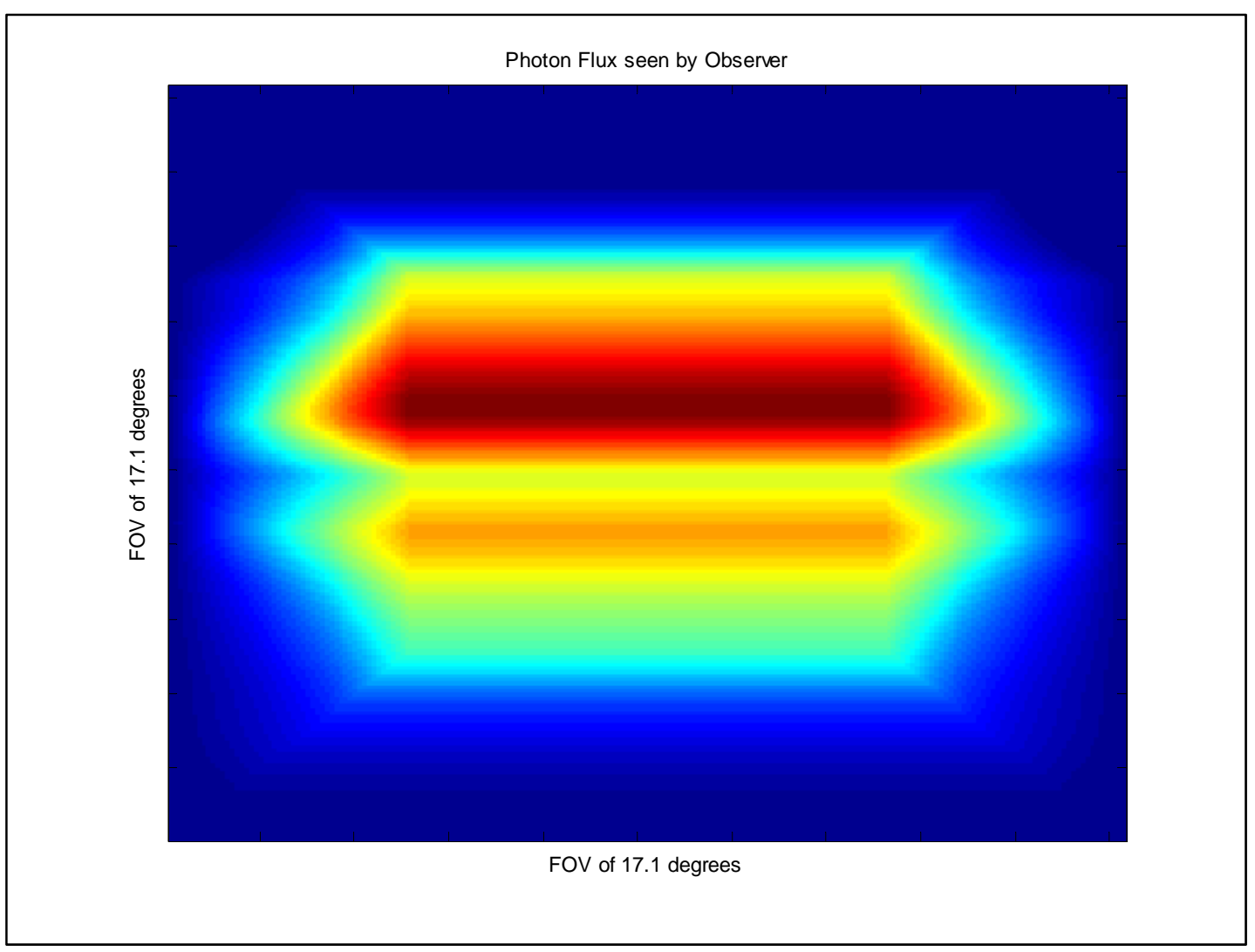

Figure 9: Photon flux as seen by an observer that is perpendicular to the sodium profile in Fig. 8 showing the double peak effect in its most extreme viewing scenario

\section{Geometry of Sodium Guidestar Models}

A sodium guidestar model of the observer being co-located with laser source was used and placed in various regions of the globe during chosen times of the year to show the performance of using a sodium guide star as it would be used from an astronomical telescope to correct for atmospheric turbulence.

For demonstration of the LEEDR tool capability, four main geometries were chosen to show the capability of viewing a sodium guide star from various aspects representative of real scenarios. The four scenarios included viewing the sodium guidestar from the laser source, from a ground based observer $100 \mathrm{~km}$ away, from an aircraft scenario $100 \mathrm{~km}$ away, and from a low earth orbiting satellite viewing the guidestar from off the main axis. 
The two ground based scenarios simulate using a guidestar as part of a co-located transmit and receive telescope or having the guidestar source attach to the side of the observing telescope, and viewing the sodium guide star from a bi-static location. For an adaptive optics system having the laser guide star source co-located with the imaging telescope is most common, but bi-static systems do exist.

The aircraft scenario simulates observing a sodium guidestar from a sensor that would be located on a C-130 aircraft or a predator drone flying near their respective maximum altitudes at a $100 \mathrm{~km}$ stand-off. The maximum altitude for both aircraft is approximately $7 \mathrm{~km}$. From this collection scenario as well as the bi-static ground based collection scenario the effects of spot elongation can start to be seen.

The last scenario is sodium guide star viewing from a satellite. This adds a unique observer feature to LEEDR and HELEEOS. It also opens up viewing of the sodium guidestar from an assortment of viewing angles that are not typically used. It may be important for researchers who conduct sodium guidestar tests to view the sodium guidestar from space as then the photon flux can be studied more closely from many angles during one engagement scenario. Utilizing all four observation scenarios allows a three dimensional view of a sodium guidestar to be created.

\section{Simulation Set-up Parameters and Assumptions}

Parameters used for engagement scenarios can be found in the Table $3 .^{10}$ 
Table 3: Parameters used for the sodium photon flux simulations that are representative of a typical sodium guide star system used for atmospheric correction ${ }^{2,6,10, \text { and } 11}$

\begin{tabular}{|c|c|}
\hline \multicolumn{2}{|c|}{ Parameters Used for Simulations } \\
\hline Parameter & Value \\
\hline Number of Grids & 100 \\
\hline Grid Resolution & 101 \\
\hline Ground Observer Altitude (km) & 0 \\
\hline Aircraft Observer Altitude (km) & 7 \\
\hline Space Observer Altitude (km) & 700 \\
\hline Detector Efficiency (\%) & 100 \\
\hline Wavelength (nm) & 589.1 \\
\hline Wave Type & Continuous \\
\hline Laser Energy (W) & 50 \\
\hline Aperture Diameter (m) & 1.5 \\
\hline Focus Distance & At Target \\
\hline Geometry & Varies for Each Scenario \\
\hline Engagement Time (s) & From OSIRIS Database, Specific for \\
Sodium Density Profile & Each Location \\
\hline Atmospheric Transmission & based on specific site and time \\
\hline
\end{tabular}

Assumptions and approximations used for input into the LEEDR and HELEEOS tool sets are as follows. It is assumed that the sodium atoms re-emit photons isotropically. This is a common approximation that is used in literature and modeling that gives close results to testing, but is likely not $100 \%$ accurate. Other factors that would contribute to non-isotropic radiation of photons from sodium atoms would include: the geomagnetic field effects, radiation pressure (recoil), and spin collisions effects. ${ }^{3,4,6,7,11,15,19, \text { and } 22}$

When choosing a location on the globe to set up an engagement scenario, the tool will filter through all the collected sodium layer data and specifically choose the closest data point. This is actual measured sodium layer profile data collected from the OSIRIS sensor at that location. This is not representative of an average global trend data point. Therefore, this can be misleading since at times extreme or random profiles were collected. This phenomenology is 
currently under study for the sporadic occurrences in the sodium layer density profile that occur. To mitigate this many points in approximately the same area can be used or manual sorting through the sodium layer density database can be performed, or sodium layer data can be manually chosen and inputted based off of a specific location sodium layer profile.

When performing photon flux calculations LEEDR or HELEEOS separates the propagation path into data screens and performs the calculations at each data point in each screen. Depending on the chosen resolution and number of screens, the accuracy and smoothness of the results are varied.

It is assumed that the $D_{2}$ transition for sodium is what is emitting the light. This was chosen because the $D_{2}$ transition is the stronger when compared to the $D_{1}$ transition. This topic is discussed in the section on atomic sodium physics. ${ }^{5}$ and 19 


\section{CHAPTER 4}

\section{RESULTS}

\section{Global Sodium Data Viewed in LEEDR}

In LEEDR there is a capability to show the monthly global sodium layer density distribution. An example of what this looks like in LEEDR is shown in Fig. 10. To create these global density distribution plots, all the measured sodium layer data from the OSIRIS sensor were taken and separated into each month. Within each month, there were thousands of data points that corresponded to various locations on earth. Within each data point the maximum density was used as a reference for that point and gridded to the globe. Grid intersections that had no collected data points were created from a linear interpolation of the closest two data points. A full mesh grid of data points was created from this and used to create a scaled overlay in red. The maximum grid point was given a transparency value of 1 , meaning it was completely opaque, and the minimum which in all cases was zero was given a transparency value of zero, making it completely transparent. ${ }^{6}$

The global sodium layer monthly plots can be found in Appendix II. Looking at the plots there are apparent features within the monthly plots. Sharp horizontal cut-off lines in the data can be seen. This is due to the lack of collection beyond those latitudes. This is expected based on using the earth's dayglow for limb-scanning measurements and the orbit that the Odin satellite was in. During certain times of the year, it was just not possible for the OSIRIS sensor to collect sodium layer data beyond certain latitudes. Another feature that can be noted is that 
some months look like there is a lot of sodium layer data and others look sparse. This is an artifact of the way the data was chosen to be displayed and how the distribution from maximum to minimum sodium density values is distributed. For the months that look like there is plentiful sodium layer densities globally present, it is just more of an even distribution across the globe with the mode of the sodium layer density values is close numerically to the maximum values. Months that look sparse, indicate that there are a few localized locations on the globe where the collected data were exceptionally high as compared to the rest of the collected data in that month globally.

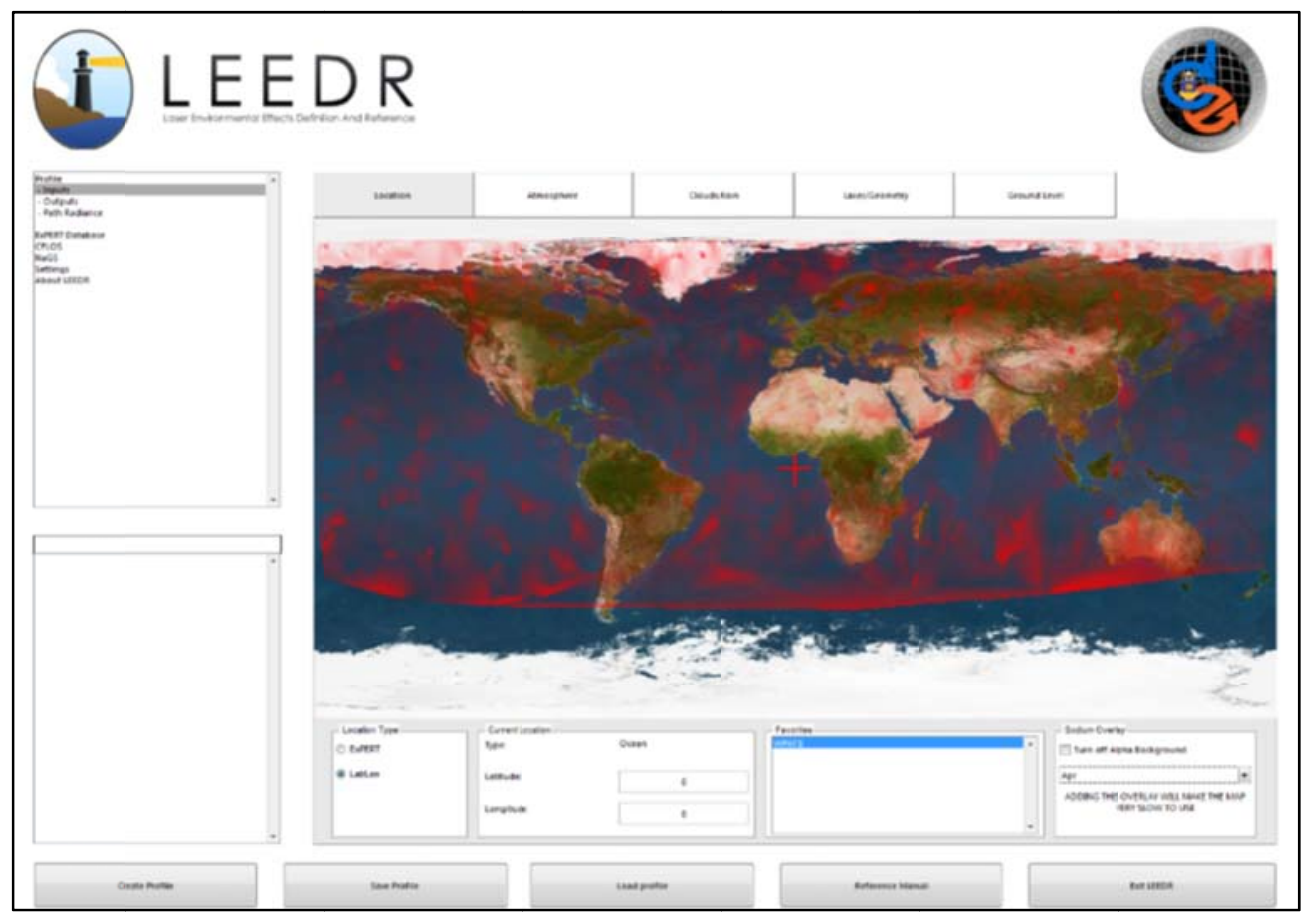

Figure 10: LEEDR plot of sodium global climatology ${ }^{6}$ 
Table 4: Sodium layer statistics for each month of collected data from the OSIRIS sensor

\begin{tabular}{|l|l|l|l|l|}
\hline \multicolumn{5}{|c|}{ Measurement Statistics of Sodium Layer } \\
\hline $\begin{array}{l}\text { Measurement } \\
\text { Group }\end{array}$ & $\begin{array}{l}\text { Number of } \\
\text { Measurements }\end{array}$ & $\begin{array}{l}\text { Max Density } \\
\text { atoms/cm }\end{array}$ & $\begin{array}{l}\text { Mean Density } \\
\text { atoms } / \mathrm{cm}^{2}\end{array}$ & $\begin{array}{l}\text { STD of the Density } \\
\text { atoms/cm }\end{array}$ \\
\hline All & 57637 & $1.2775 \mathrm{e} 10$ (July) & $6.9484 \mathrm{e} 8$ & $5.2789 \mathrm{e} 8$ \\
\hline January & 5875 & $6.0760 \mathrm{e} 9$ & $5.4854 \mathrm{e} 8$ & $3.8192 \mathrm{e} 8$ \\
\hline February & 4778 & $8.5742 \mathrm{e} 9$ & $5.2900 \mathrm{e} 8$ & $4.2826 \mathrm{e} 8$ \\
\hline March & 4648 & $8.1368 \mathrm{e} 9$ & $1.0035 \mathrm{e} 9$ & $7.9588 \mathrm{e} 8$ \\
\hline April & 3489 & $2.8550 \mathrm{e} 9$ & $6.6698 \mathrm{e} 8$ & $3.1490 \mathrm{e} 8$ \\
\hline May & 4537 & $5.6988 \mathrm{e} 9$ & $5.5114 \mathrm{e} 8$ & $3.2146 \mathrm{e} 8$ \\
\hline June & 2799 & $9.8208 \mathrm{e} 9$ & $5.5608 \mathrm{e} 8$ & $4.0488 \mathrm{e} 8$ \\
\hline July & 3223 & $1.2775 \mathrm{e} 10$ & $6.1726 \mathrm{e} 8$ & $4.8108 \mathrm{e} 8$ \\
\hline August & 5227 & $6.7846 \mathrm{e} 9$ & $7.0314 \mathrm{e} 8$ & $3.6762 \mathrm{e} 8$ \\
\hline September & 5480 & $8.1524 \mathrm{e} 9$ & $9.2782 \mathrm{e} 8$ & $6.0076 \mathrm{e} 8$ \\
\hline October & 6181 & $1.1921 \mathrm{e} 10$ & $9.8996 \mathrm{e} 8$ & $6.9498 \mathrm{e} 8$ \\
\hline November & 5229 & $5.1612 \mathrm{e} 9$ & $6.6162 \mathrm{e} 8$ & $4.6210 \mathrm{e} 8$ \\
\hline December & 6171 & $5.4328 \mathrm{e} 9$ & $4.7352 \mathrm{e} 8$ & $3.0320 \mathrm{e} 8$ \\
\hline
\end{tabular}

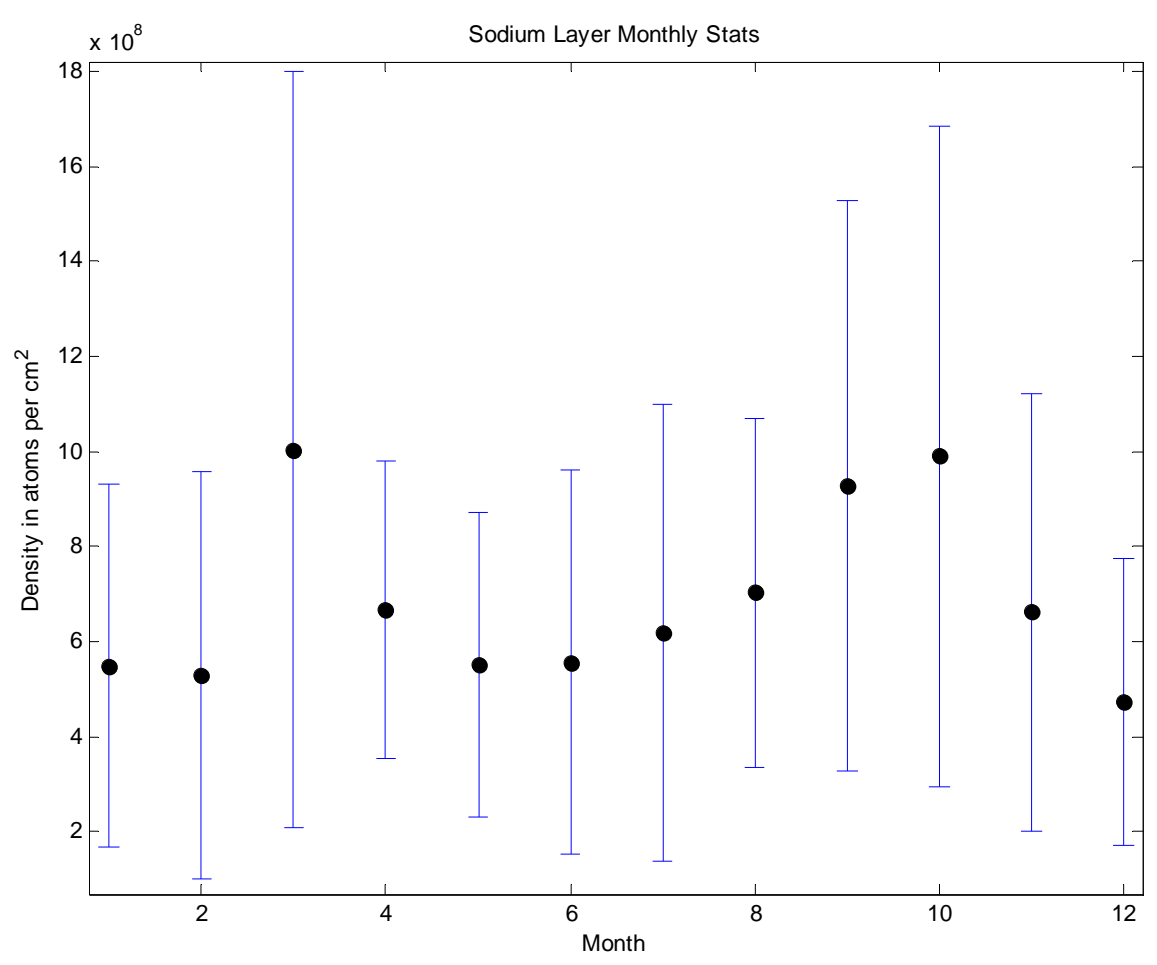


Figure 11: Monthly sodium layer mean density and standard deviation for the earth. The black points are the monthly mean values and the blue bars show the standard deviation of measured values for that month

Trends in the sodium layer density for each month as described by the maximum, mean, and standard deviation can be seen in Table 4 and Fig. 11 . When comparing the mean sodium layer density from the OSIRIS sensor data used in LEEDR, collected from 2004 to 2011 to traditional sodium layer density theory, it follows a similar trend with a few differences. In the OSIRIS data used in LEEDR there are two main high density peaks, one in March and one in the September/October timeframe. Mean densities during this time are around $1 \times 10^{9}$ atoms per $\mathrm{cm}^{2}$ and the maximum densities during these times are around $1.2 \times 10^{10}$ atoms per $\mathrm{cm}^{2}$. Comparing this to traditional global density theory, the maximum is only in the October/November timeframe and reaches values from $3.5 \times 10^{9}$ to $8 \times 10^{9}$ atoms per $\mathrm{cm}^{2}$. When looking at the standard deviation it can be seen that there is a large amount of variability in the density values. A lot of this is dependent on the location on earth of the collected data. As presented by Dr. Plane, the sodium layer density varies greatly both from month to month and across latitudes. Looking at both traditional theory and the LEEDR plots, during winter months latitudes in the northern hemisphere appear to have greater sodium column densities. While during the summer, the southern latitudes appear to have greater sodium column densities. ${ }^{6}$

\section{Photon Flux from Ground based Simulation Scenarios}

In Fig. 12, the sodium profile chosen for simulations can be seen. This profile was chosen because of its uniqueness and double peak. Based on some of the scenarios presented this feature displays the capability of modeling the photon flux in LEEDR and HELEEOS. Of note, 
this is just an example scenario profile chosen, in LEEDR and HELEEOS there are over 57,000 different specific profiles that can be selected based on time and location.

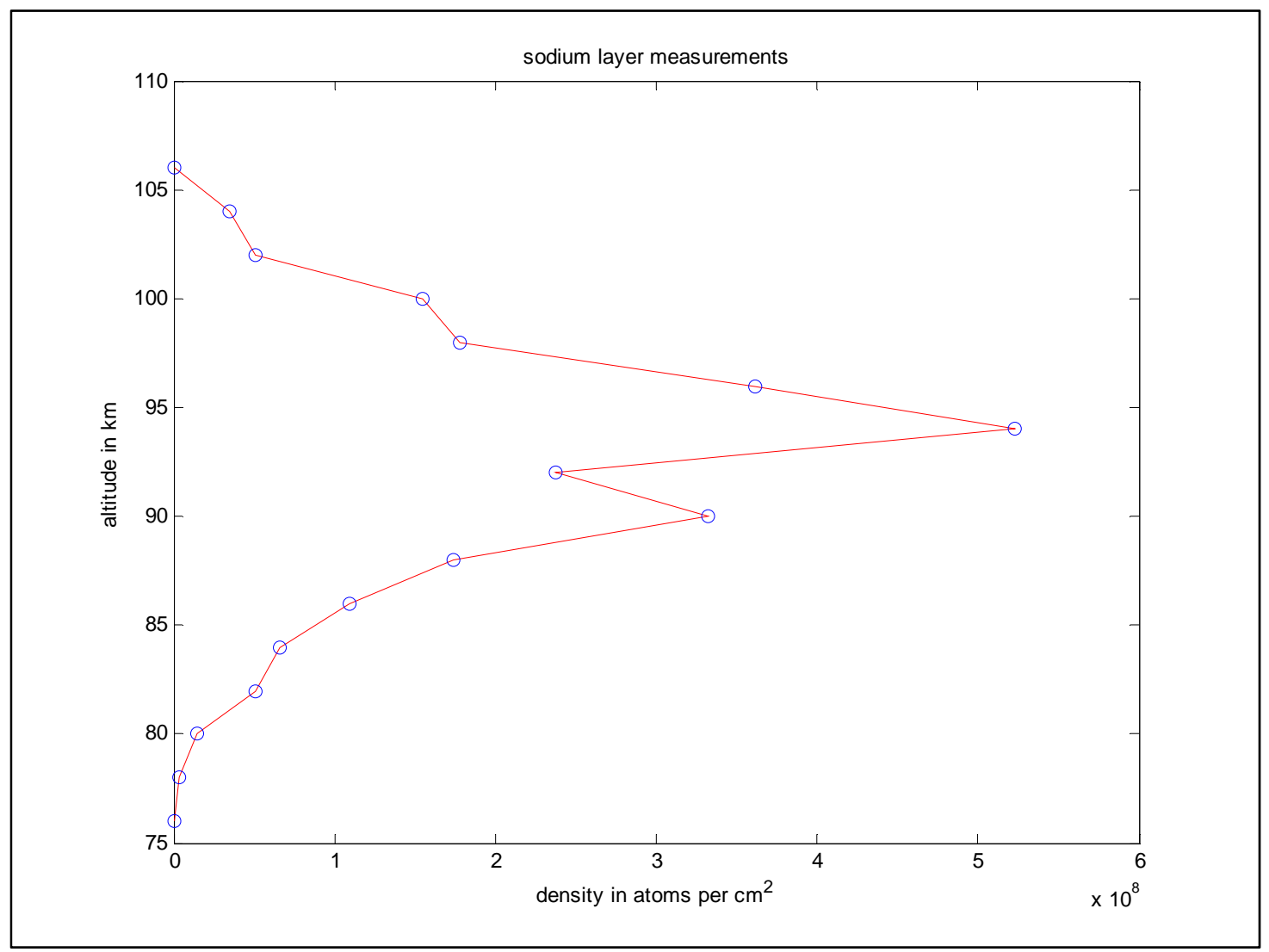

Figure 12: Sodium layer profile data used for simulation scenarios presented

Fig. 13 shows the photon flux as seen from an observer that is on the ground and 100 $\mathrm{km}$ from the source of the sodium guidestar. This represents a ground based bi-static collection on a sodium guidestar. In Fig. 13, the effects of spot elongation can start to be noticed, although they are minimal across the field of view of the observer. Spot elongation will start to cause problems when using a sodium guidestar in conjunction with a Shack-Hartmann wave front sensor. The Shack-Hartmann wave front sensor places spots on each lenslet. If the spots are elongated, then it can create errors in determining the centroid location and hence the displacement of each spot. ${ }^{21}$ 


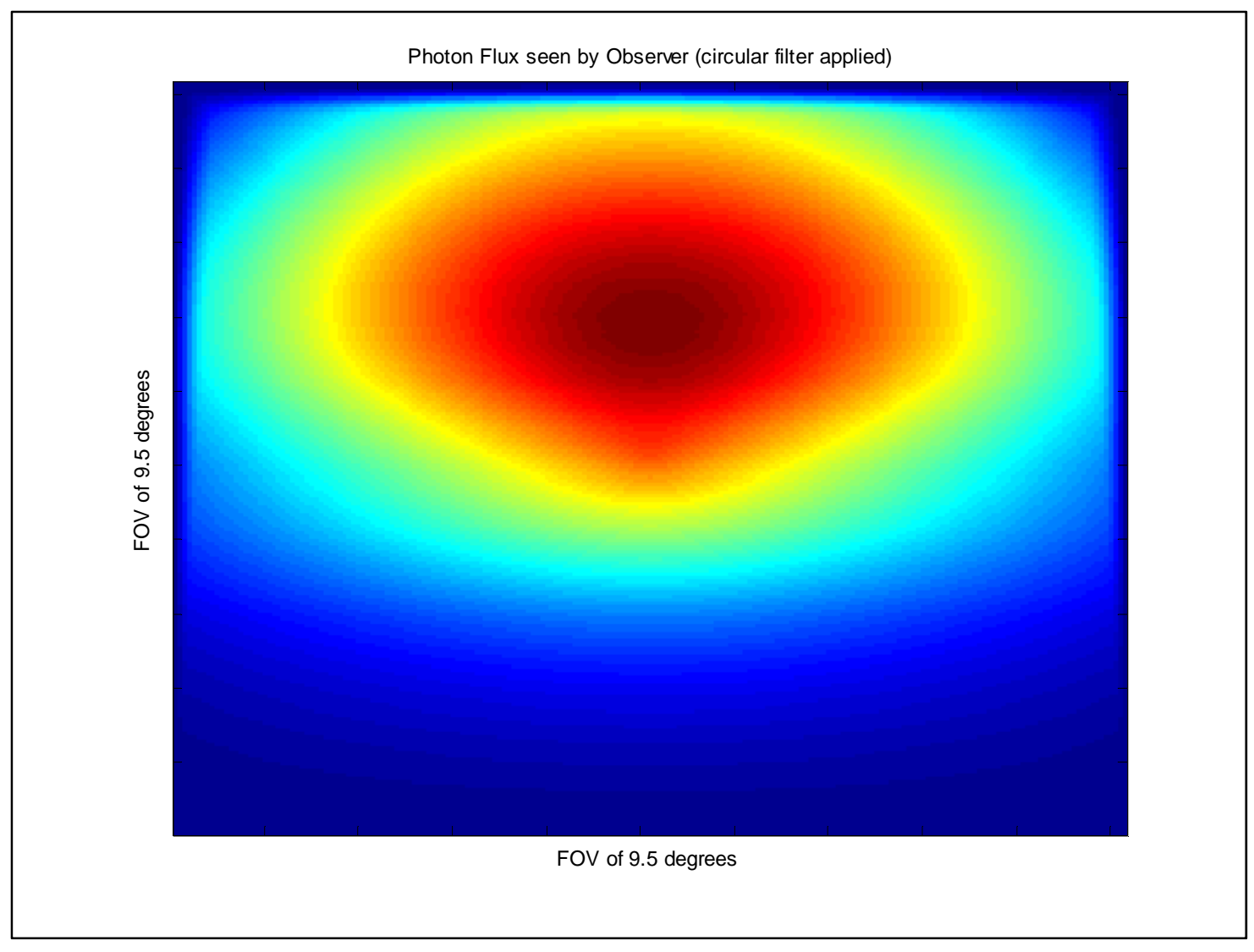

Figure 13: Photon flux profile from a ground based observer with a 9.5 degree FOV at a $100 \mathrm{~km}$ standoff showing a near Gaussian profile with slight spot elongation due to the field viewing geometry

\section{Photon Flux from Air based Simulation Scenarios}

Fig. 14 shows the photon flux as seen from an observer that is on an aircraft flying at an altitude of $7 \mathrm{~km}$ and $100 \mathrm{~km}$ from the source of the sodium guidestar. This is a flying altitude that is representative of near maximums for a C-130 aircraft or a predator drone. Since there is relatively minimal difference between this scenario and a ground based scenario the photon flux plots look very similar. Of note, it was assumed that the aircraft was stationary for this test setup. Having a moving sensing platform introduces a new set of criteria to consider such as air turbulence induced from air flows around the aircraft and sensor, tracking considerations, and jitter induced from flight movement. 


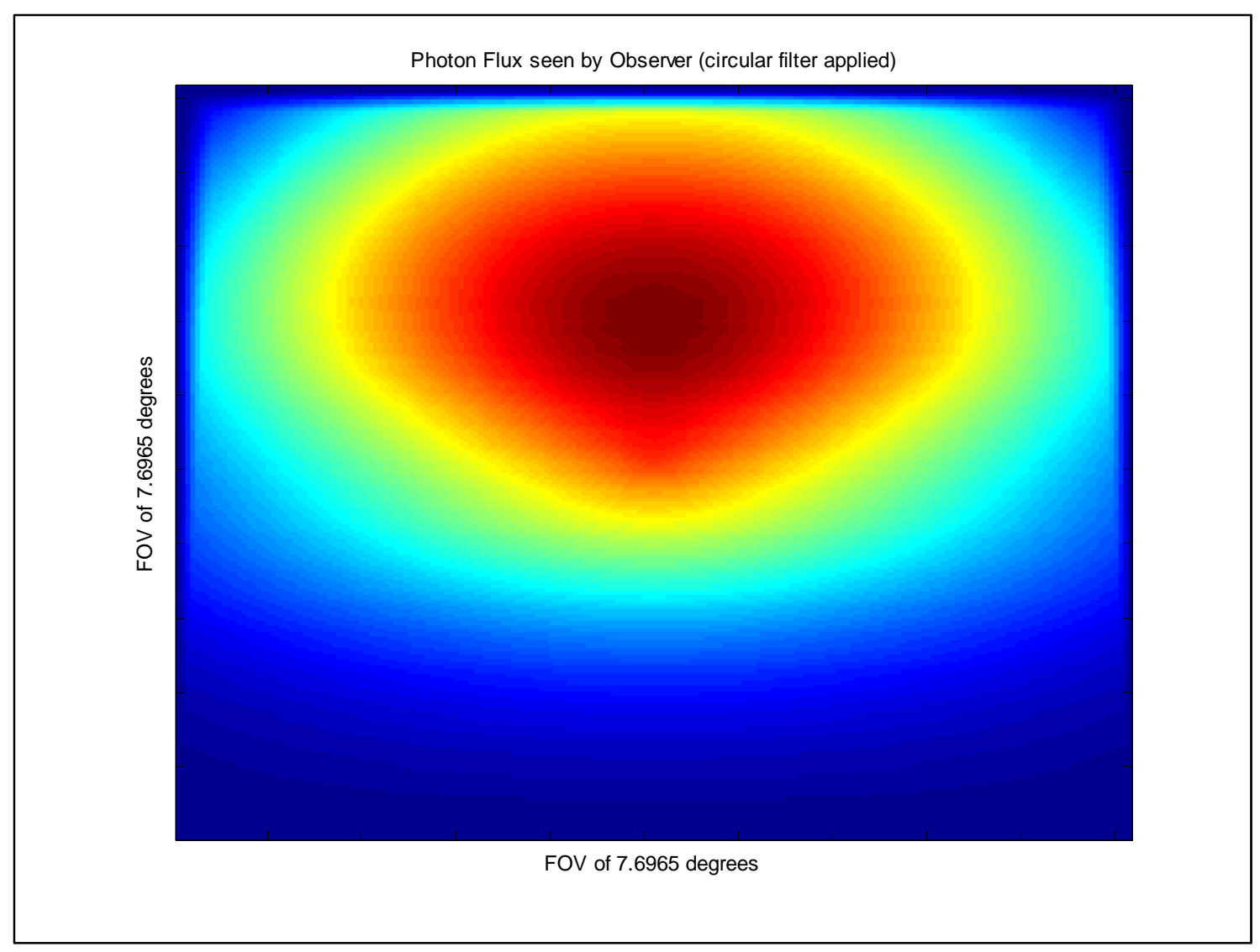

Figure 14: Photon flux profile from an air based observer at an altitude of $7 \mathrm{~km}$ with a 7.6965 degree FOV at a $100 \mathrm{~km}$ standoff showing a near Gaussian profile with slight spot elongation due to the field viewing geometry

\section{Photon Flux form Space based Simulation Scenarios}

Figures 15 and 16 show the photon flux as seen from an observer that is in space at an altitude of $700 \mathrm{~km}$ and $100 \mathrm{~km}$ and $1000 \mathrm{~km}$ from the source of the sodium guidestar as measured from the satellite sub-points. A $700 \mathrm{~km}$ altitude is represnetitive of a low earth orbit satellite. Also, for these calculation only a one-way transmission was used since the second beam path does not travel through the atmosphere, but instead travels upward toward the satellite observor. Only having a $100 \mathrm{~km}$ satellite sub-point standoff distance creates a geometry that can be viewed as nearly overhead, but not in the main beam path. This is important as the sodium layer saturates using only about 1-3 percent of the laser energy and 
the rest just passes through into space. Being $100 \mathrm{~km}$ off the spot is seen as circular and the effects of spot elongation are not present. Moving to a standoff distance of $1000 \mathrm{~km}$, spot elongation effects can be seen. Also, due to the earth calculations used in LEEDR and HELEEOS and the inputs currently available, it can be hard to determine the correct orientation and field of view of the observor to get the whole sodium guidestar spot in the image with the nessessary resolution.

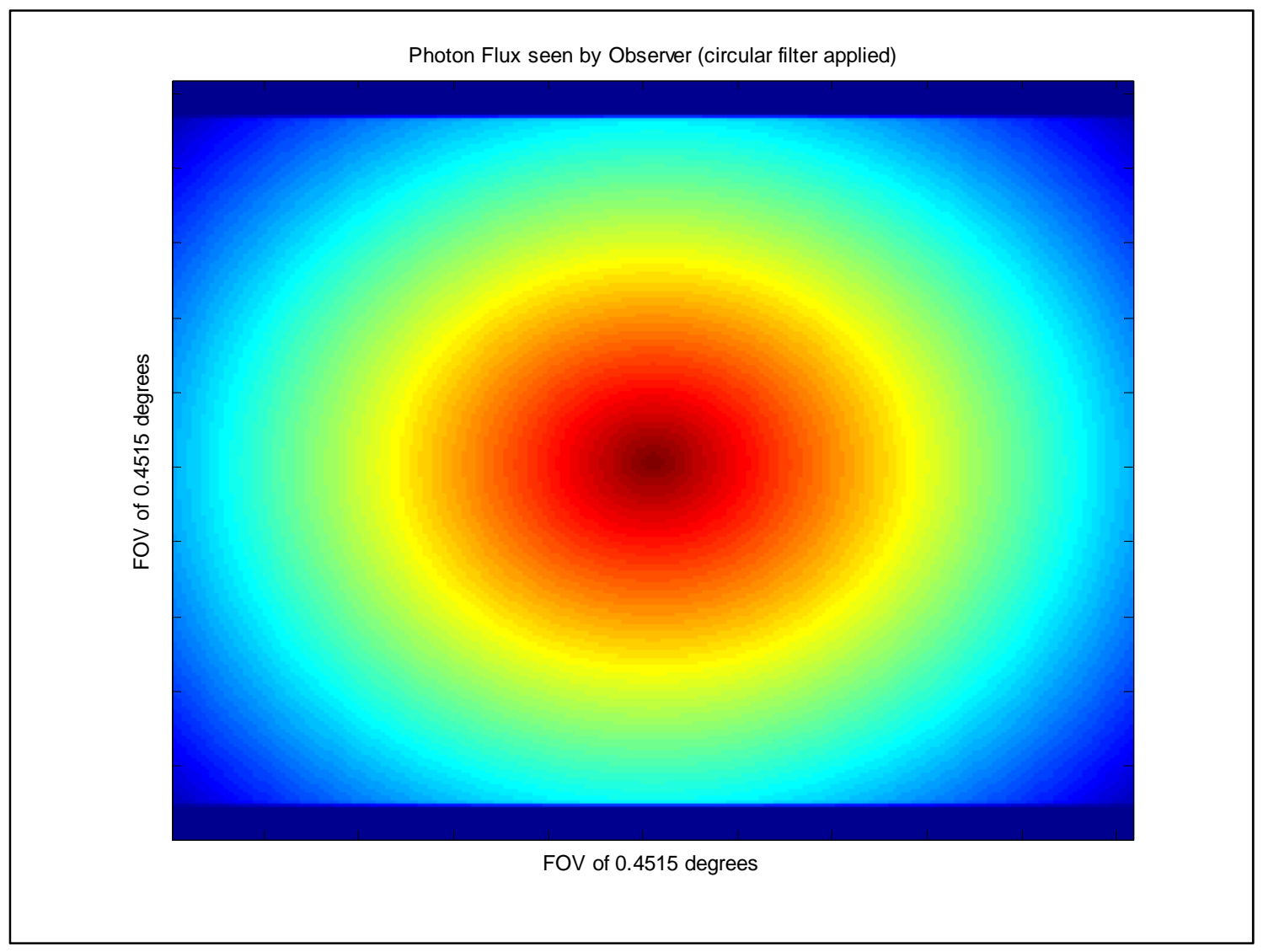

Figure 15: Photon flux profile from a space based observer at an altitude of $700 \mathrm{~km}$ with a 0.4515 degree FOV at a $100 \mathrm{~km}$ satellite sub-point standoff showing a Gaussian profile since the geometry of the standoff is far enough away that spot elongation is unnoticeable 


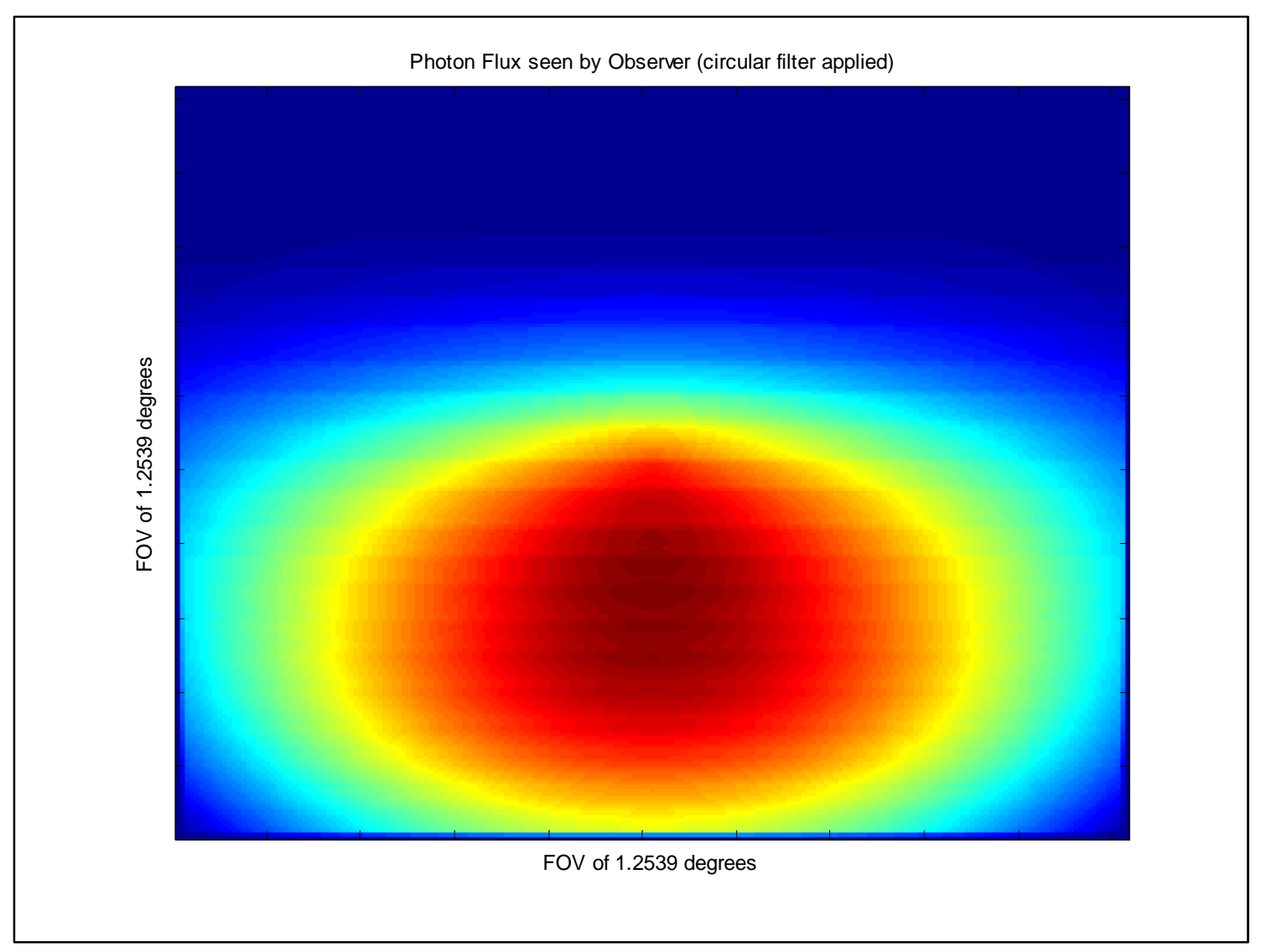

Figure 16: Photon flux profile from a space based observer at an altitude of $700 \mathrm{~km}$ with a 1.2539 degree FOV at a $1000 \mathrm{~km}$ satellite sub-point standoff showing a profile where the spot elongation is evident, but the double peak structure is not yet observable

Fig. 17 illustrates what a sodium guidestar would be seen as in its most extreme case of being viewed from space directly from the side. Here the whole structure of the sodium profile can be seen, as noted by the double peak structure which matches the sodium density profile. For this simulation, an arbitrary standoff distance was used as it would appear to be the same from any distance as long as the resolution and field of view were adjusted proportionally. It is important to notice that the profile structure is present along the central portions of the field of view. On the left and right edges of the plot localized at the two main peaks, the photon flux appears to have a spike and decrease more rapidly than near the center of the plot. This is due 
to a stronger isotropic radiation from the two peaks and compared to being away from the peaks.

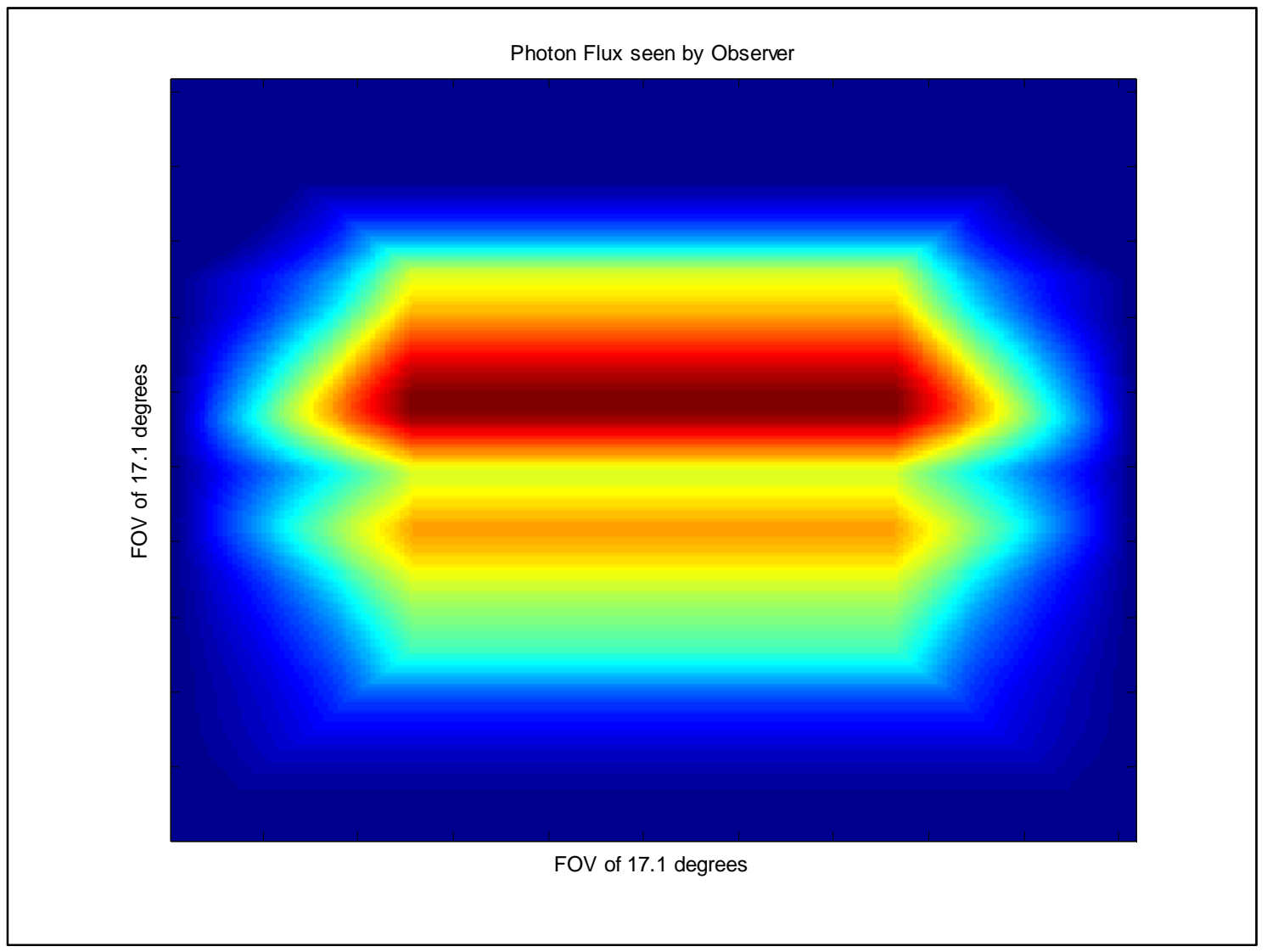

Figure 17: Photon flux profile from a space based observer representative of any location that could view the sodium layer directly from the side capturing the entirety of the sodium layer absorption and resonant scattering showing the effects on the photon flux from the double peak sodium column density

\section{Optimal land based locations for a Sodium Guide Star System}

When looking at the global climatology of the sodium layer it is important to understand the trends in each region specifically since the sodium layer density changes have a large impact on the guidestar performance. Thirty-five regions on earth were chosen that are representative of the major areas of each of the seven continents for a study on the feasibility of using a guidestar system. For a sodium guidestar to be effect as a reference in an adaptive optic system 
it needs to be equivalent to a magnitude 12 star; this corresponds to needed a sodium density of $8.8436 \times 10^{8}$ atoms per $\mathrm{cm}^{2}$ as presented in the methodology section. It is important to know that this number of sodium atoms is not absolute, but only representative of a typical adaptive optic system. If a more advanced or capable adaptive optic system is available to sodium density necessary to create a sufficiently bright sodium guidestar can be less. Data was gathered for each site corresponding to all collected sodium layer density data within 10 degrees of latitude and longitude from a central point in the region and then categorized into a monthly trend. Data tables and charts for this can be found in the Appendix III.

When looking at the trend for North America it appears that the sodium layer reaches its highest values in the fall and winter months with lows in the summer months. For the most part, during the summer none of the North American sites reach the requirement for the adaptive optic system presented. This means for an adaptive optic system, a more capable system would be needed to meet the minimum requirements to perform atmospheric corrections needed for imaging scientific objects of interest in space. There are also fairly large swings in the sodium densities on a month to month basis which create a wide range of variability even within each month's dataset. When analyzing sites in North America across latitudes it appears that the southern sites like the Eastern and Western United States have relatively higher density counts as compared to Alaska and Canadian sites. Reference the data table and charts in the appendix for specifics on monthly values at each site.

When looking at the sodium density trends in Asia, they follow a similar trend to those in North America with the highest values in the fall and winter months and lowest values in the summer. Overall, the sites in Asia appear to be closer to the minimum sodium density needed with the location of Eastern Russia, Northern Russia, Central Russia, and Western China reaching sodium density values well above the minimum for the fall and winter months. Locations like 
Indonesia, Saudi Arabia and India are below the minimum for most of the year except a few months where they barely make it above the minimum value. Reference the data table and charts in the appendix for specifics on monthly values at each site.

When looking at the sodium density trends for locations in South America, most sites for most of the year are below the minimum requirement for an adaptive optic system presented. Eastern Brazil goes above the minimum brightness requirement in two months, February and May. The Central Argentina location is above the threshold for three months of the year, May, June and November. The southern South American site is above the threshold for two months, August and October. Northern South America is above for two months, May and October. Finally, central South America is above threshold for only one month, November. Overall, the trend of the sodium layer density for sites in South America appears to be sporadic in nature and is unfavorable for a typical sodium guidestar system for most of the year. Reference the data table and charts in the appendix for specifics on monthly values at each site.

All the European locations follow the same trend in sodium layer density data. This is likely due to their close proximity and small regional size. They all are above the required threshold from approximately August through April based on collected data. This is the same as known sodium layer theory that is historically presented. Reference the data table and charts in the appendix for specifics on monthly values at each site.

The regions in Africa, Australia and Antarctica appear to follow a different trend in sodium layer density than the rest of the world. They appear to be at a low from January to May and reach higher values in the late summer and fall. Sodium layer densities are below the required minimum for most of the year, except for two months where it barely gets above the threshold, March and August. This would make an adaptive optic system unfavorable at the South Pole 
that utilized a sodium guidestar. Reference the data table and charts in the appendix for specifics on monthly values at each site.

\section{Note on Special Applications of Sodium Data in LEEDR and HELEEOS}

With the addition of the sodium layer data to LEEDR and HELEEOS it adds a unique and very specific way to view a sodium guidestar's performance. LEEDR and HELEEOS have the capability to ingest real collected atmospheric data from testing or a specific site to create an accurately representative atmosphere transmission calculation. The sodium layer database adds this same type of capability for sodium layer density profiles on a global scale that are specific to a time and location. It is possible to match the atmospheric profile with the sodium layer profile in both time and space to create an actual model of the full atmosphere used in a sodium laser guidestar. This will be extremely beneficial to scientists analyzing test data. HELEEOS also models beam propagation very uniquely in that it takes into account for a shift from a Gaussian profile beam into a crescent moon shaped beam due to thermal blooming and other atmospheric effects. This will apply to the propagation of higher energy sodium guidestar laser that are tightly focused and can create an accurate model three dimensional space of a sodium guidestar's performance. ${ }^{6 \text { and } 7}$ 


\section{CHAPTER 5}

\section{CONCLUSION}

\section{Conclusions from Work Presented}

Using the sodium layer data collected from the OSIRIS sensor adds a new capability to the LEEDR and HELEEOS toolsets. These atmospheric and laser propagation tools now account for another set of particulates in the atmosphere, sodium atoms. In addition, the NAGS interface allows for the study of the performance of a sodium guidestar system. The specific collected sodium layer data from a location and time can be used in conjunction with the existing LEEDR and HELEEOS architecture to create real scenario input parameters used to calculate the photon flux from a guidestar as viewed from a chosen observer location. This functionality is available across the entire globe and is unique to the LEEDR and HELEEOS toolsets when compared to other existing sodium guidestar tools available.

Available in the LEEDR and HELEEOS toolsets is the ability to view the global climatology of the sodium layer data from seven years of collected data from the OSIRIS sensor. The climatology is presented on a monthly timescale and the distribution trends can be seen. By observation, it can be seen that during the winter months the latitudes in the northern hemisphere have greater sodium densities, and during the summer months, the southern latitudes have greater sodium densities. Looking at the monthly statistics, differences are present as compared to traditional theory. Those being the relative high point in March and a one month shift in the primary peak density in the September to October timeframe. Also 
present is a large variability in the sodium layer density which is commonly mentioned in literature but not quantified to the extent presented in this paper.

Using the NAGS interface in LEEDR and HELEEOS photon flux calculations from a sodium guidestar can be quantified and studied. This paper presented the common real world scenarios that are now capable of being researched used in conjunction with real collected global data. The scenarios presented were all for a ground based sodium guidestar system that was collected upon from sensors that were co-located with the laser source, on the ground at a significant standoff distance from the laser source, on an air based platform at a significant standoff distance from the laser source, and on a space based platform representing a low earth orbit satellite. These are just common scenarios that represent current real world sodium guidestar scenarios. Using the NAGS interface, a completely geometrically variable system can be set up for the laser source, target, and observer. These scenarios are all quantified by a point by point photon flux calculation and a visual of the photon flux profile as seen by the observer.

The global feasibility of a sodium guidestar system was presented by selecting 35 locations on earth representing all major regions of all continents. A back calculation from a star brightness magnitude of 12 using assumed sodium guidestar system parameters representative of a typical sodium guidestar system was used to define the necessary sodium layer abundance needed to have an effective sodium guidestar that could be used for atmospheric correction. At each location this sodium layer abundance was observed on a monthly basis by taking in all collected data points within a 10 degree latitude and longitude region centered at each location. From this no one location on earth proved to be the best location for all times during the year. Comparatively, most locations were at best meeting the needed threshold for about half the year. With most locations on earth performing best during the late fall and winter months, except for some locations in Africa and South America which performed best during the summer 
months. It can be concluded that to have a sodium guidestar system capable of producing a brightness magnitude star of at least 12 for 12 months of the year a more advanced sodium guidestar system would be needed. Parameters that could lead to this include larger optics, use of a pi polarized light, quantized effects of the geomagnetic field, and pumping both the $D_{1}$ and $D_{2}$ sodium lines simultaneously.

\section{Future Development and Applications}

To increase the global data set of sodium layer data new collected data would be needed either from a satellite based sensor in a different orbit that is more favorable to collecting sodium density measurements in the northern latitudes in winter months or supplement the current data set with sodium layer lidar measurements from sites in the northern latitudes. This would help fill in the gaps in collected data both in the special and temporal domains. In addition, for specific adaptive optics sites using sodium guide stars, localized measurements taken frequently would create a more accurate picture of the sodium profile relative to those specific locations.

The HELEEOS software could also be increased in capability by adding system level adaptive optic components. Adding in a Shack-Hartmann wave front sensor and a deformable mirror application would allow for HELEEOS to calculate how a corrected beam would propagate from an actual adaptive optic system utilizing a guidestar in a real scenario. Also, adding effects from the earth's magnetic field and scattering effects from near collisions would increase the fidelity of the sodium guidestar model. For this paper it was assumed that the sodium guidestar radiate isotropically. However there are a few studies suggesting this is not always true and there are a few factors that contribute to this such as the earth's magnetic field. 


\section{BIBLIOGRAPHY}

1. Ageorges, N. and Dainty C. Laser Guide Star Adaptive Optics for Astronomy. Kluwer Academic Publishers, Dordrecht, Netherlands. 2000. Print.

2. Air Force Research Laboratory Directed Energy Directorate. "Starfire Optical Range at Kirtland Air Force Base, New Mexico". 03 September 2012. http://www.kirtland.af.mil/library/factsheets/factsheet.asp?id=15868. 05 March, 2014.

3. Brennan, T. J. and P. H. Roberts. AOTools User's Guide. The Optical Sciences Company, 2008.

4. Drummond, Jack, Novtny, Steve, Denman, Craig, Hillman, Paul, Telle, John, Moore Gerald, Eickhoff, Eric, and Fugate, Robert. "Sodium Guidestar Radiometry Results from the SOR's 50W Fasor". Starfire Optical Range, Directed Energy Directorate, Air Force Research Laboratory, Kirtland AFB, New Mexico. PDF.

5. Fan et al. "Satellite Measurements of the Global Mesospheric Sodium Layer." Atmospheric Chemistry and Physics.2007. PDF.

6. Fiorino, S.T., R.M. Randall, R.J. Bartell, A. Downs, P. Chu, and C.W. Fan, 2011: "Climate Change: Anticipated Effects on High Energy Laser Weapon Systems in Maritime Environments" J. Appl. Meteor. Climatol. Vol 50, No. 1, pp. 153-166.

7. Fiorino, S.T., R.M. Randall, M.F. Via, and J.L. Burley, 2014: "Validation of a UV-to-RF highspectral-resolution atmospheric boundary layer characterization tool" J. Appl. Meteor. Climatol. Vol 53, No. 1, pp. 136-156.

8. Fugate, Robert Q. "Prospects for Benefits to Astronomical Adaptive Optics from US Military Programs." Adaptive Optical Systems Technology, Proceedings of SPIE Vol. 4007. 2000. PDF.

9. Fugate, Robert Q. "The Starfire Optical Range 3.5-m Adaptive Optical Telescope." Large Ground-Based Telescopes, Proceedings of SPIE Vol. 4837. 2003. PDF.

10. Fussen, D. at all. "A Global Climatology of the Mesospheric Sodium Layer from GOMOS Data during the 2002-2008 Period". Atmospheric Chemistry and Physics 10, 9225-9236, 2010. 1 October 2010. PDF.

11. Gardner et al. "Seasonal Variations of the Na and Fe Layers at the South Pole and Their Implications for the Chemistry and General Circulation of the Polar Mesosphere." Journal of Geophysical Research, VOL. 110, 17 May 2005. PDF. 
12. Gumbel et al. "Retrieval of Global Mesospheric Sodium Densities from the Odin Satellite." Geophysical Research Letters VOL. 34, 2007. PDF.

13. Herriot, Glen at al. "Sodium Laser Guide Star Issues: Modeling and Mitigation". Herzberg Institute of Astrophysics, University of Victoria, 29 October 2007. PDF.

14. Hillman, Paul, Drummond, Jack, Denman, Craig, and Fugate, Robert. "Simple Model, Including Recoil, for the Brightness of Sodium Guide Stars Created from CW Single Frequency Fasors and Comparison to Measurements." Adaptive Optics System, Proceedings of SPIE Vol. 7015. 2008. PDF.

15. Holzlohner, Rochester, Calia, Budker, and Higbie. "Optimization of CW Sodium Laser Guide Star Efficiency." Faculty Research and Publications, Bucknell University. 2010. PDF.

16. Humphreys et al. "Sodium-Layer Synthetic Beacons for Adaptive Optics." The Lincoln Laboratory Journal. Volume 5, Number 1, 1992. PDF.

17. Jeys, Thomas H. "Development of a Mesospheric Sodium Laser Beacon for Atmospheric Adaptive Optics." The Lincoln Laboratory Journal VOL 4, November 1991. PDF.

18. Kibblewhite, Edward. "The Physics of the Sodium Laser Guide Star: Predicting and Enhansing the Photon Returns". University of Chicago. PDF.

19. Kyrola, E., Blanot, L. at al. "GOMOS Algorithm Theoretical Basis Document". December 2012. PDF.

20. Max, Claire. "Lecture 11 - Introduction to Laser Guide Stars". Astro 289, UC Santa Cruz. February 12, 2013. PDF.

21. Moussaoui, N. at all. "Effect of the Geomagnetic Field on the Intensity of Sodium Laser Guide Stars. Facilty of Physics, University of Sciences and Technology Houari Bomediene, Algiers, Algeria and European Southern Observatory, Munchen, Germany. PDF.

22. Moussaoui, N. at al. "Statistics of the Sodium Layer Parameters at Low Geographic Latitude and its Impact on Adaptive-Optics Sodium Laser Guide Star Characteristics". Astronomy and Astrophysics 511,A31 (2010). 6 November 2009. PDF.

23. M. R. Whiteley, E. P. Magee, and A. M. Ngwele, "Scaling for High Energy Laser and Relay Engagement (SHaRE) User Guide," MZA Associates, Dayton OH, (2011)

24. Pique, Jean-Paul and Farinotti, Sebastien. "An Efficiant Modeless Laser for a Mesospheric Sodium Laser Guide Star". Laboratoire de Spectrometrie Physique, Universite Joseph Fourier. PDF.

25. Plane, John. "A Reference Atmosphere for the Atomic Sodium Layer". School of Chemistry, University of Leeds, Leeds, UK. PDF.

26. Plane, John. "A time-resolved model of the mesospheric Na Layer: constraints on the meteor input function". Atmospheric Chemistry and Physics, 4, 627 (2004). PDF.

27. Plane et al. "First Observations of the Global Sodium Layer." Geophysical Research Abstracts, Vol. 7, 2005. PDF. 
28. Plane, John at al. "Meteoric Smoke - Where on Earth is it?" University of Leeds, School of Mathematics and Physical Sciences. CEDAR Prize Lecture 2007. PDF.

29. Steck, Daniel A. "Sodium D Line Data". Theoretical Division (t-8), Los Alamos National Laboratory, Los Alamos, NM. 14 October 2003. PDF.

30. Telle, John, Milonni, Peter, and Fugate, Robert. "Update on 589-nm Sodium Guidestar Pump Laser Requirements." Adaptive Optical Systems Technology Preceding's of SPIE Vol. 4007, 2000. PDF.

31. Tyson, Robert K. Principles of Adaptive Optics. Boca Raton, FL: CRC, 2011. Print.

32. Van Zandt, N.R., S.T. Fiorino, and K.J. Keefer, 2013: "Enhanced, fast-running scaling law model of thermal blooming and turbulence effects on high energy laser propagation" Opt. Express, Vol. 21, Iss. 12, pp. 14789-14798.

33. Vishnu Prasanth, P. at al. "Lida Observations of the Sodium Layer over low Latitude, Gadanki (13.50 N. 79.20 E): Seasonal and Nocturnal Variations". Annales Geophysicae, 27, 3811-3823, 2009. 7 October 2009. PDF.

34. Whiteley, Dr. Robert at al. "Improving Laser-Guide Star AO Observations Via Mesospheric Sodium Enhancement." PDF. 


\section{APPENDIX I}

\section{GLOBAL SODIUM DATA PLOTS}

In appendix I, all the sodium data is presented as sorted by month. The data is displayed as sodium density in atoms per square centimeter vs altitude in kilometers. From these plots, one can see how the profile of the sodium layer changes from month to month based on the collected OSIRIS data from 2004 to 2011.

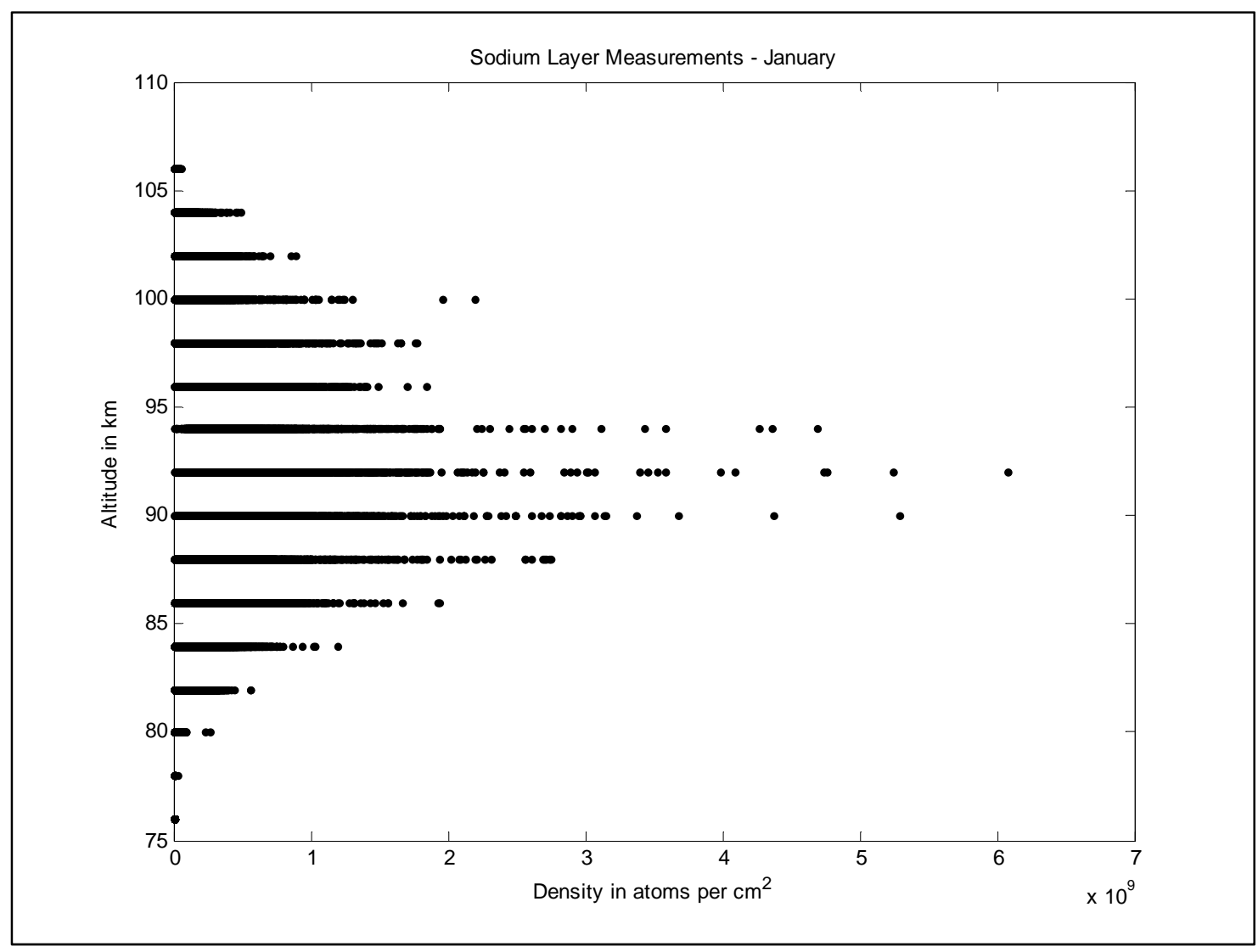

Figure 18: Sodium layer density vs altitude for January for all collected data across the globe 


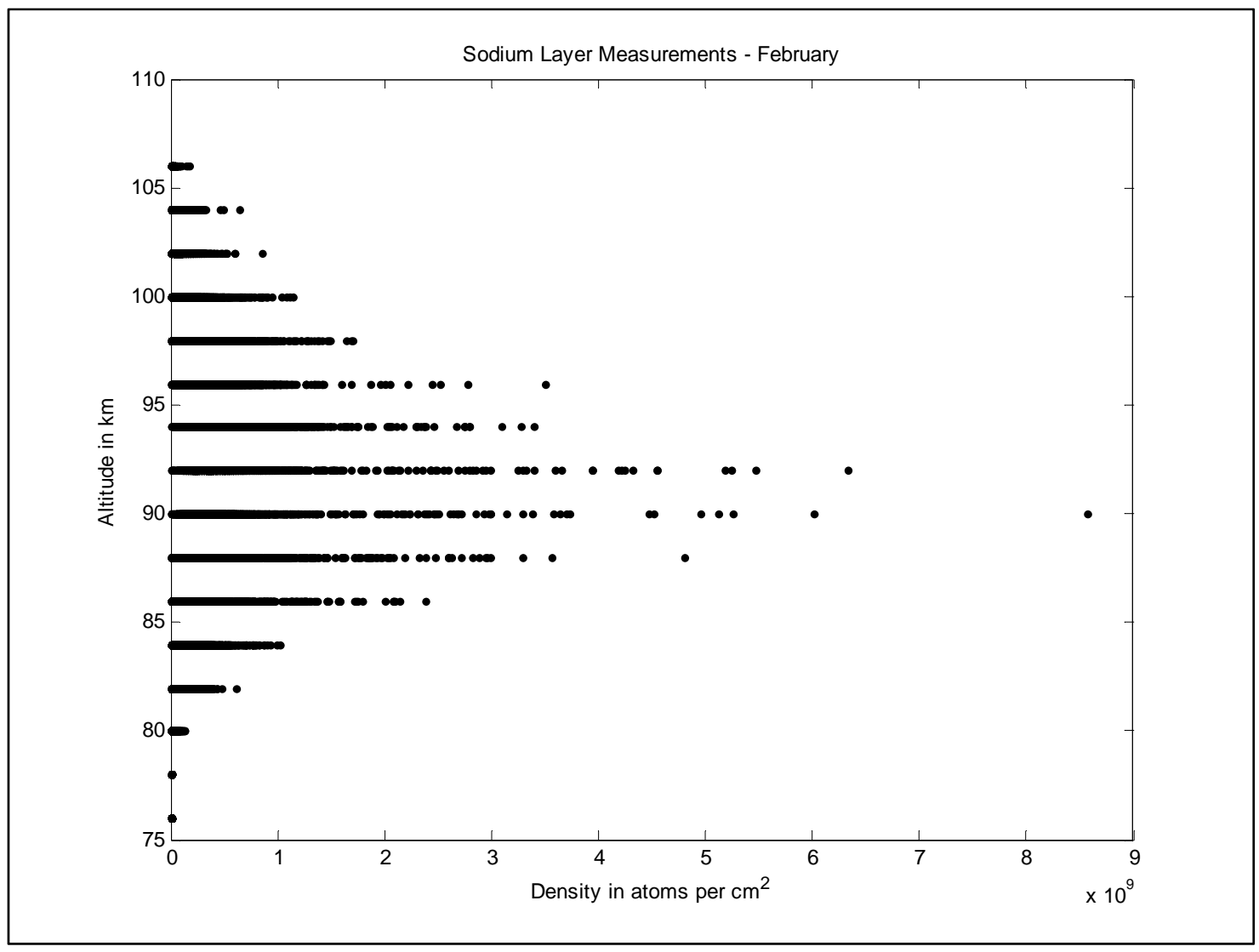

Figure 19: Sodium layer density vs altitude for February for all collected data across the globe 


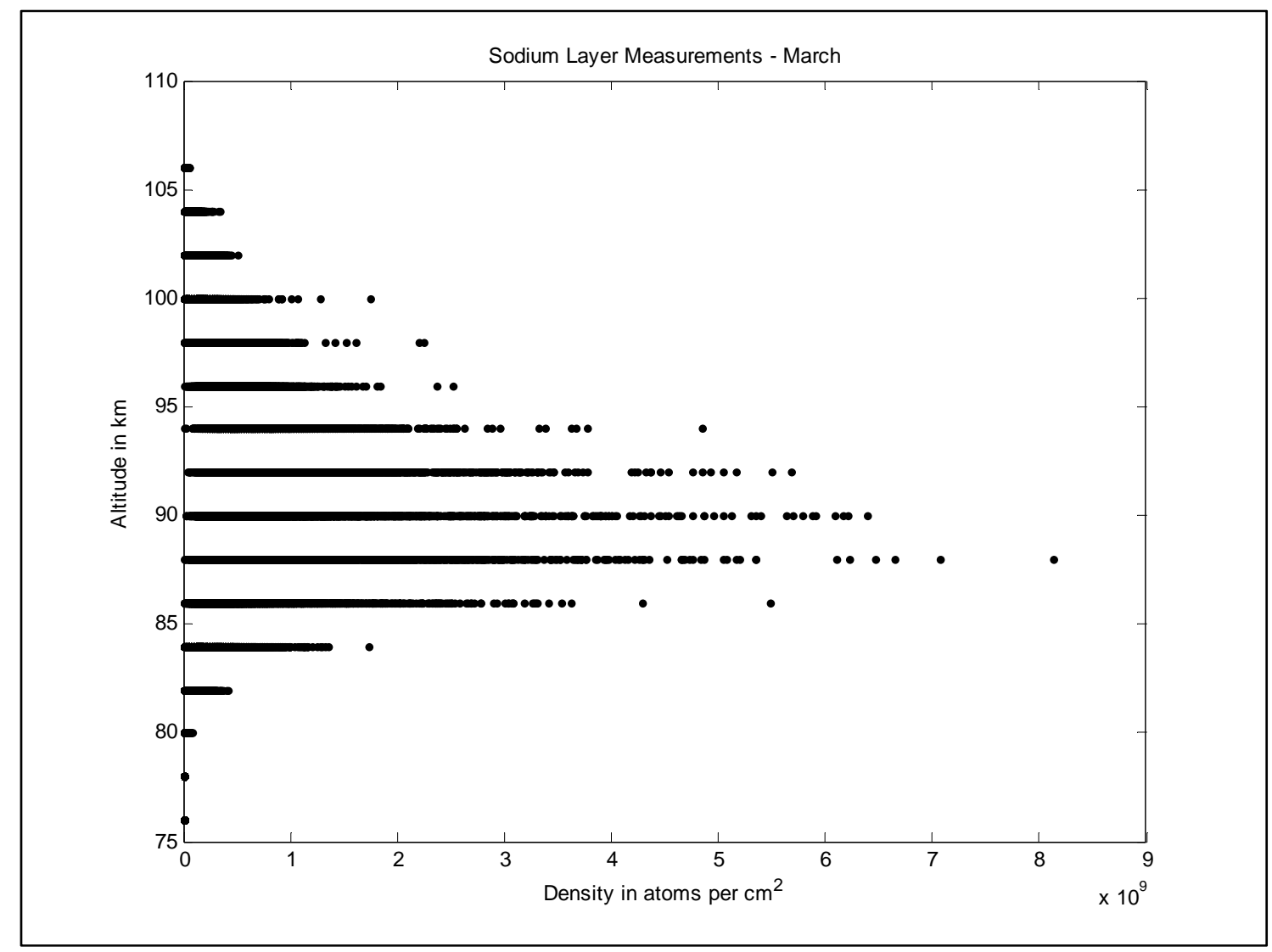

Figure 20: Sodium layer density vs altitude for March for all collected data across the globe 


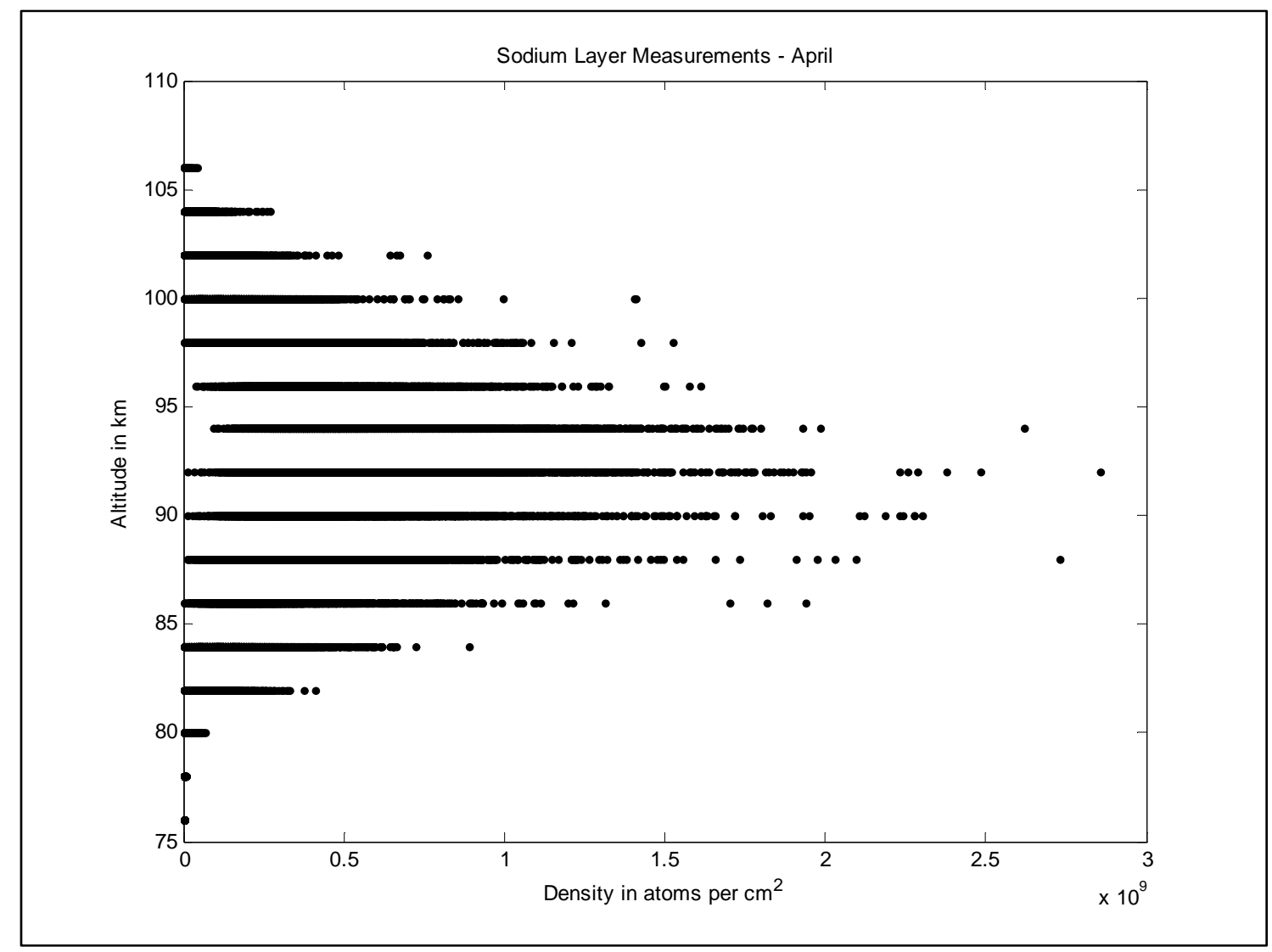

Figure 21: Sodium layer density vs altitude for April for all collected data across the globe 


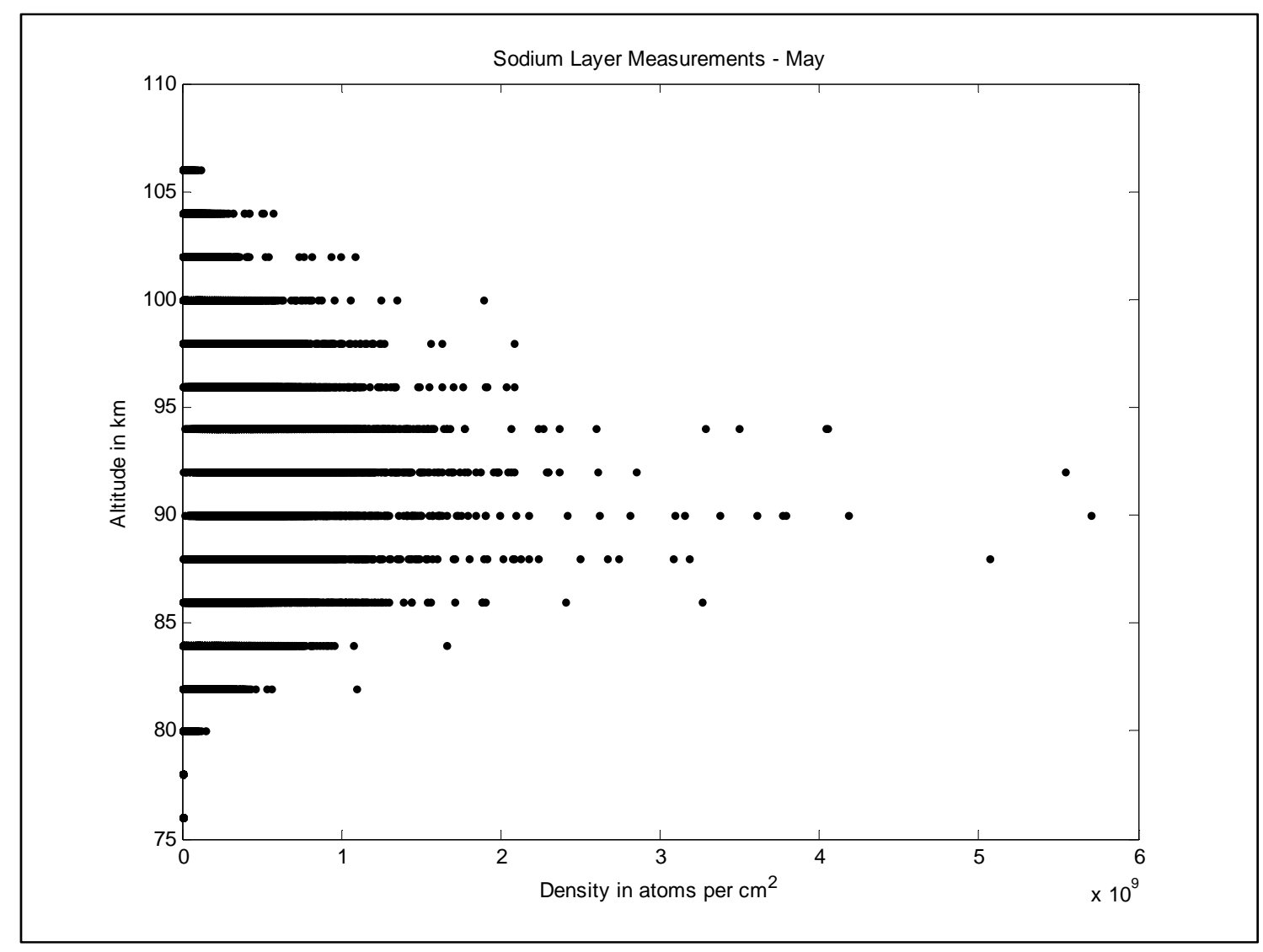

Figure 22: Sodium layer density vs altitude for May for all collected data across the globe 


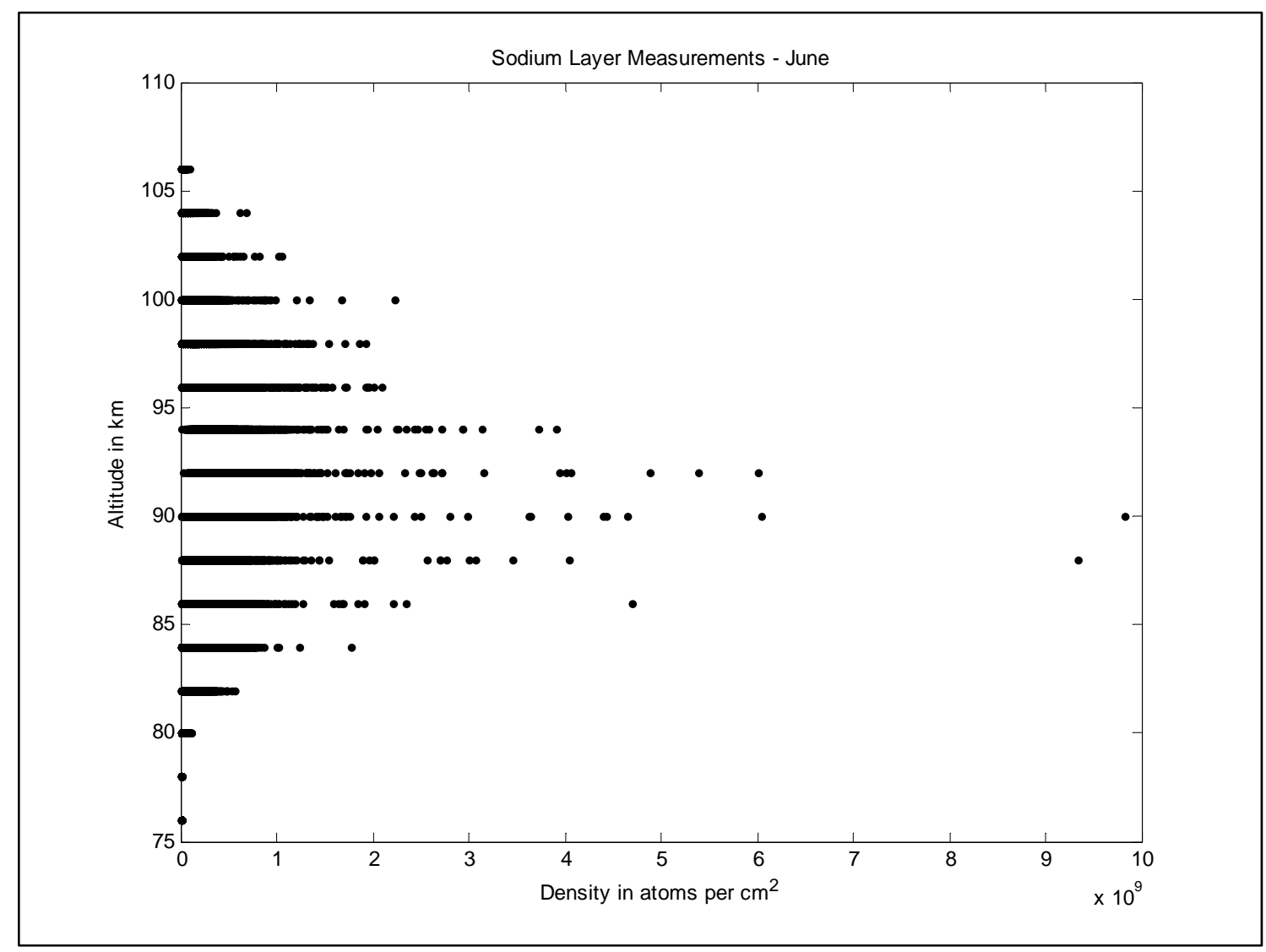

Figure 23: Sodium layer density vs altitude for June for all collected data across the globe 


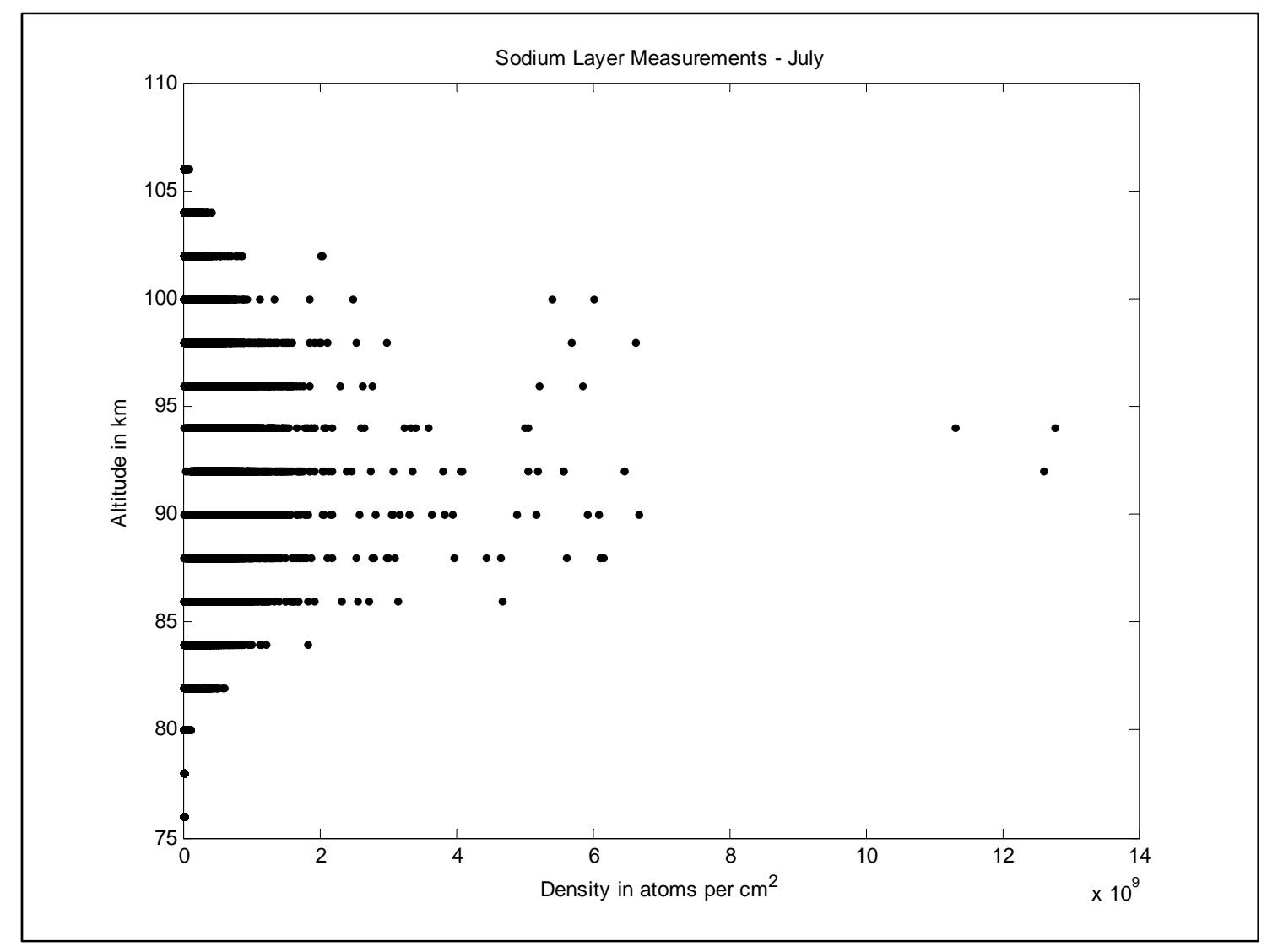

Figure 24: Sodium layer density vs altitude for July for all collected data across the globe 


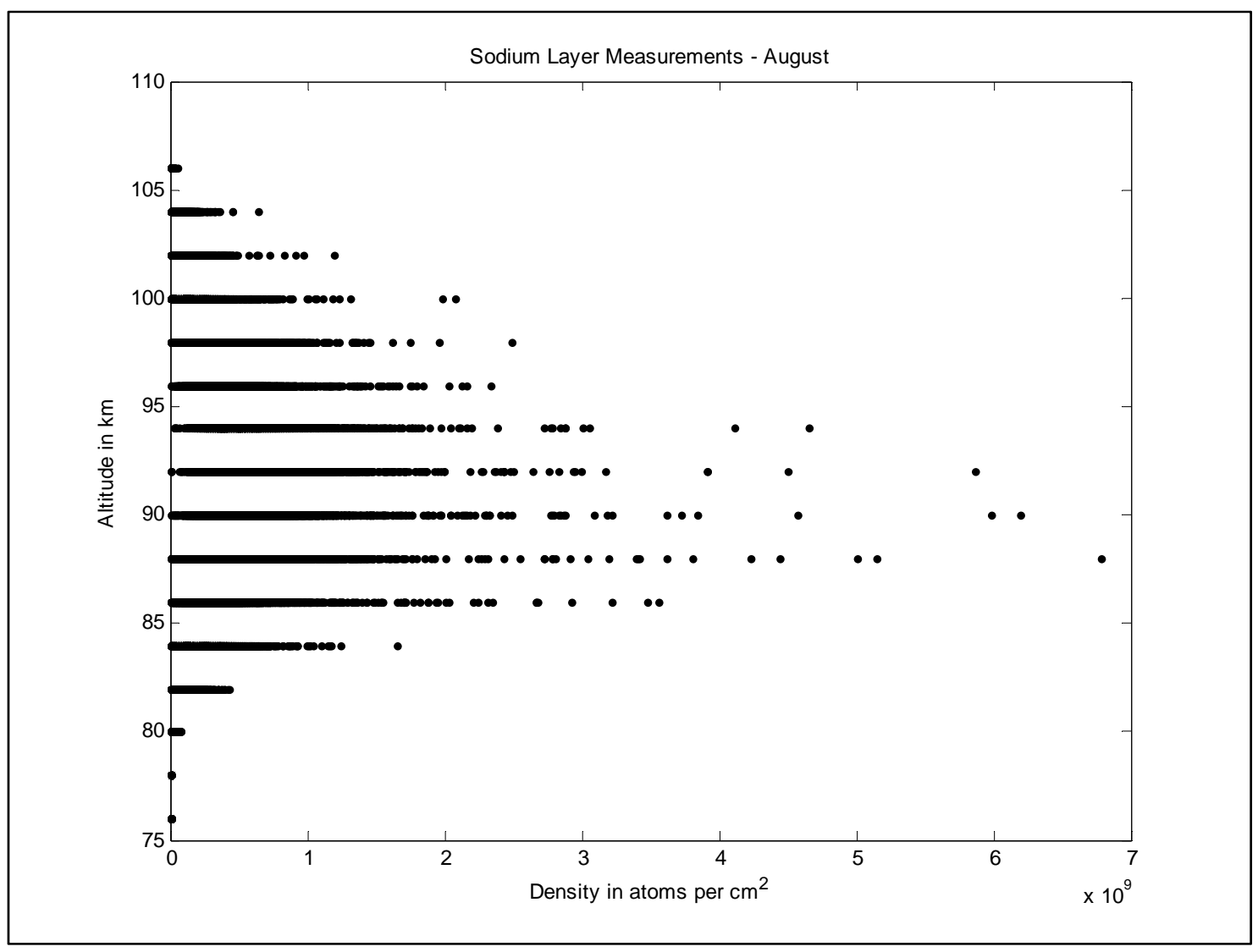

Figure 25: Sodium layer density vs altitude for August for all collected data across the globe 


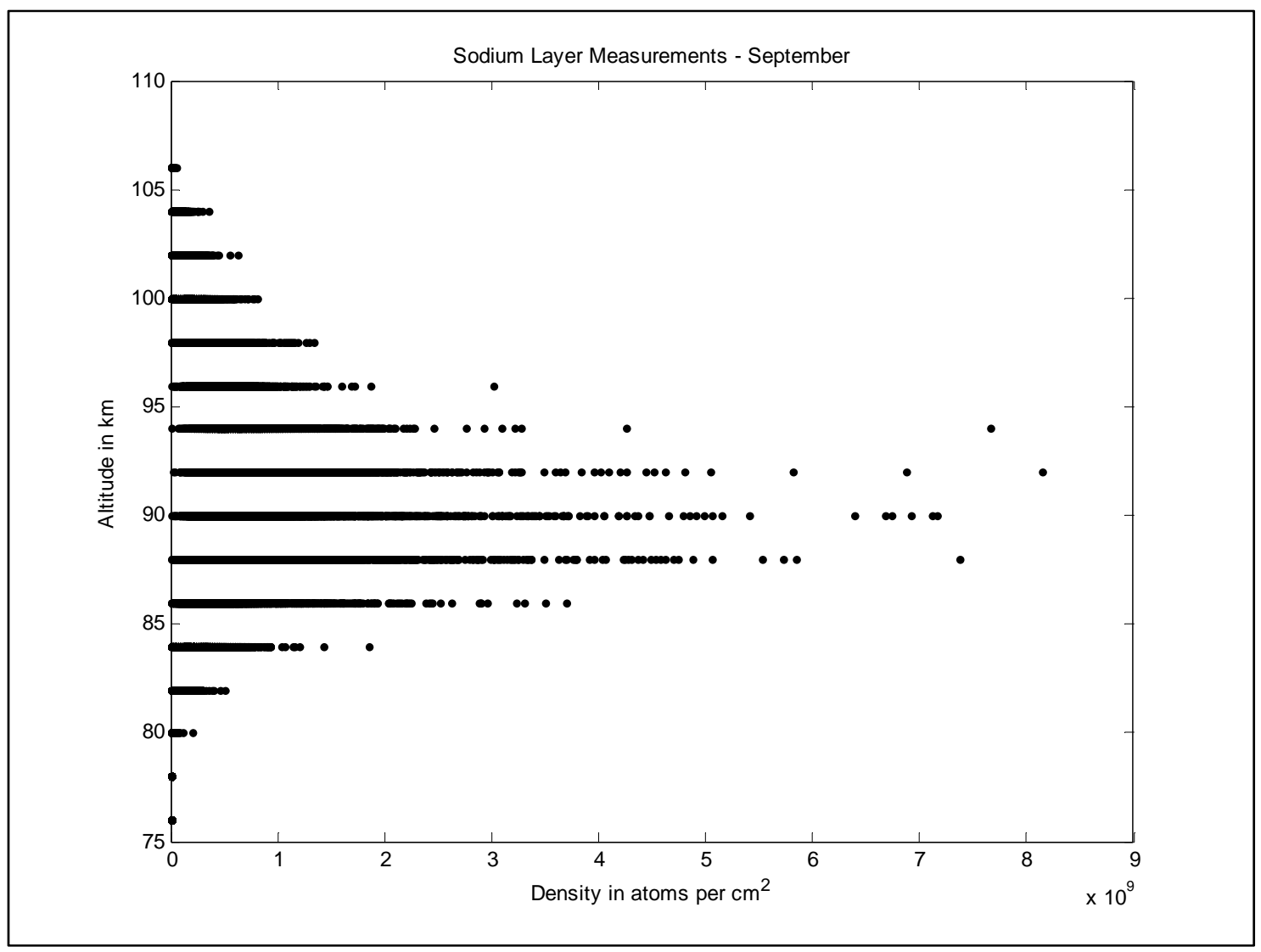

Figure 26: Sodium layer density vs altitude for September for all collected data across the globe 


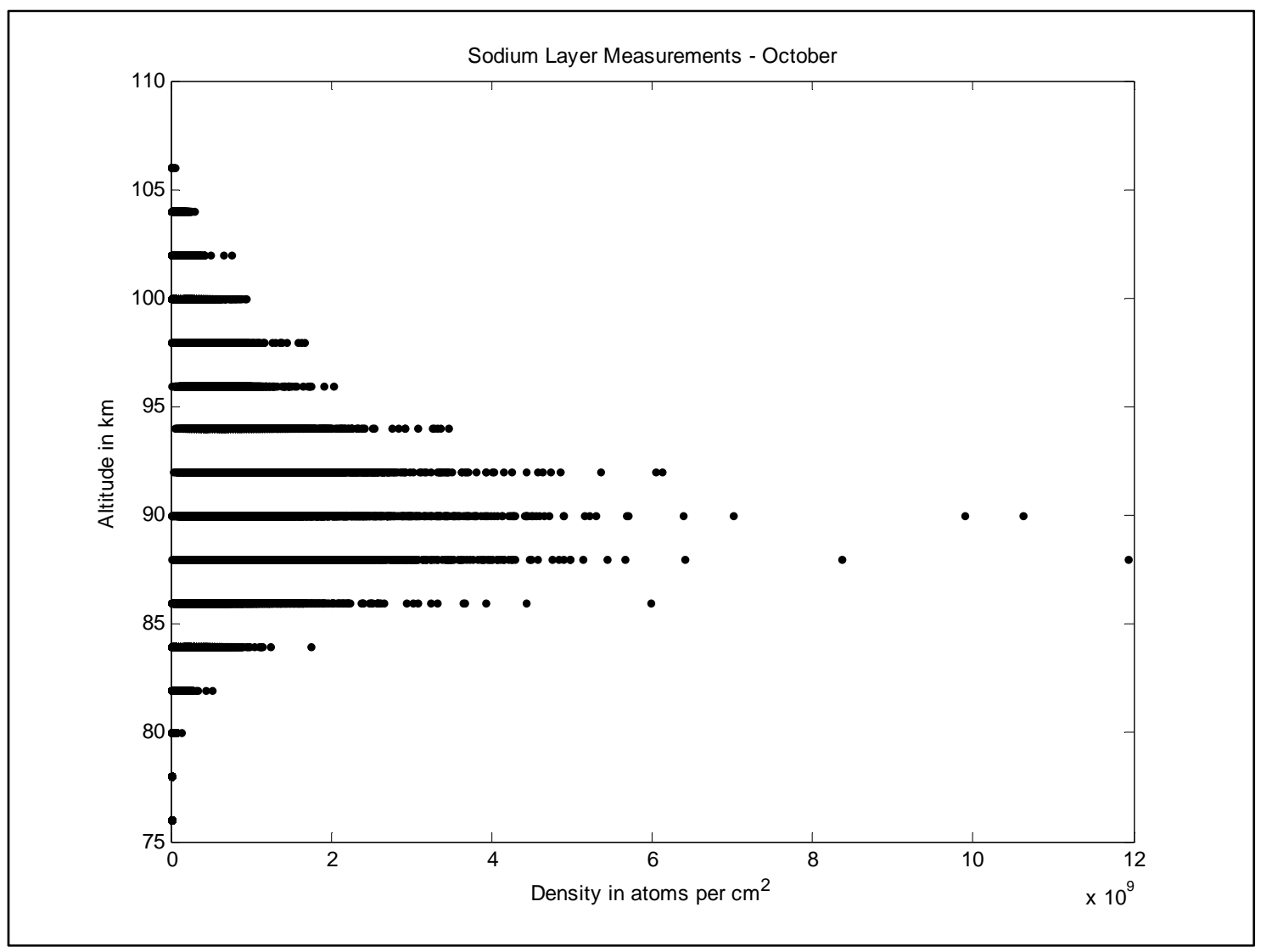

Figure 27: Sodium layer density vs altitude for October for all collected data across the globe 


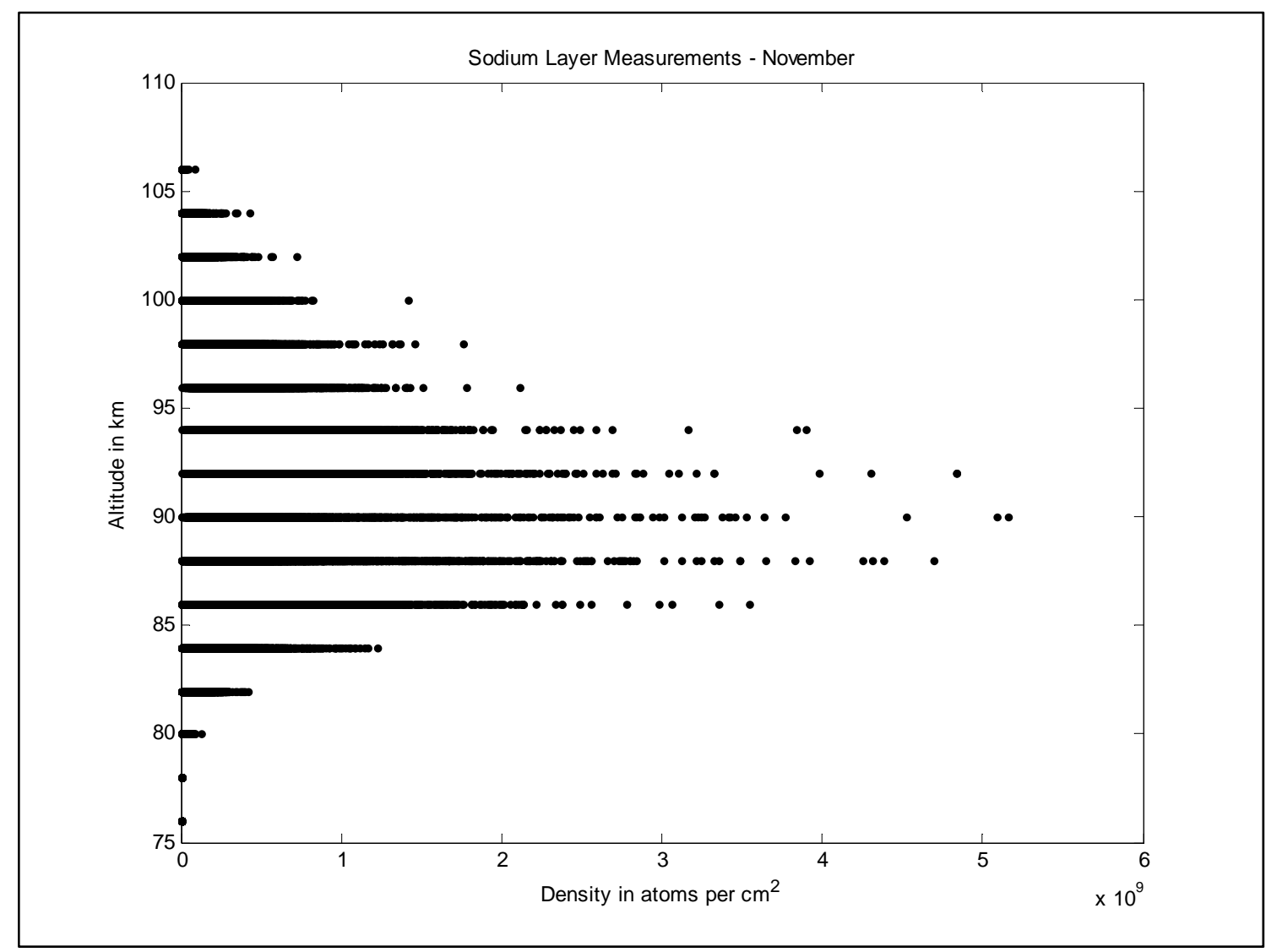

Figure 28: Sodium layer density vs altitude for November for all collected data across the globe 


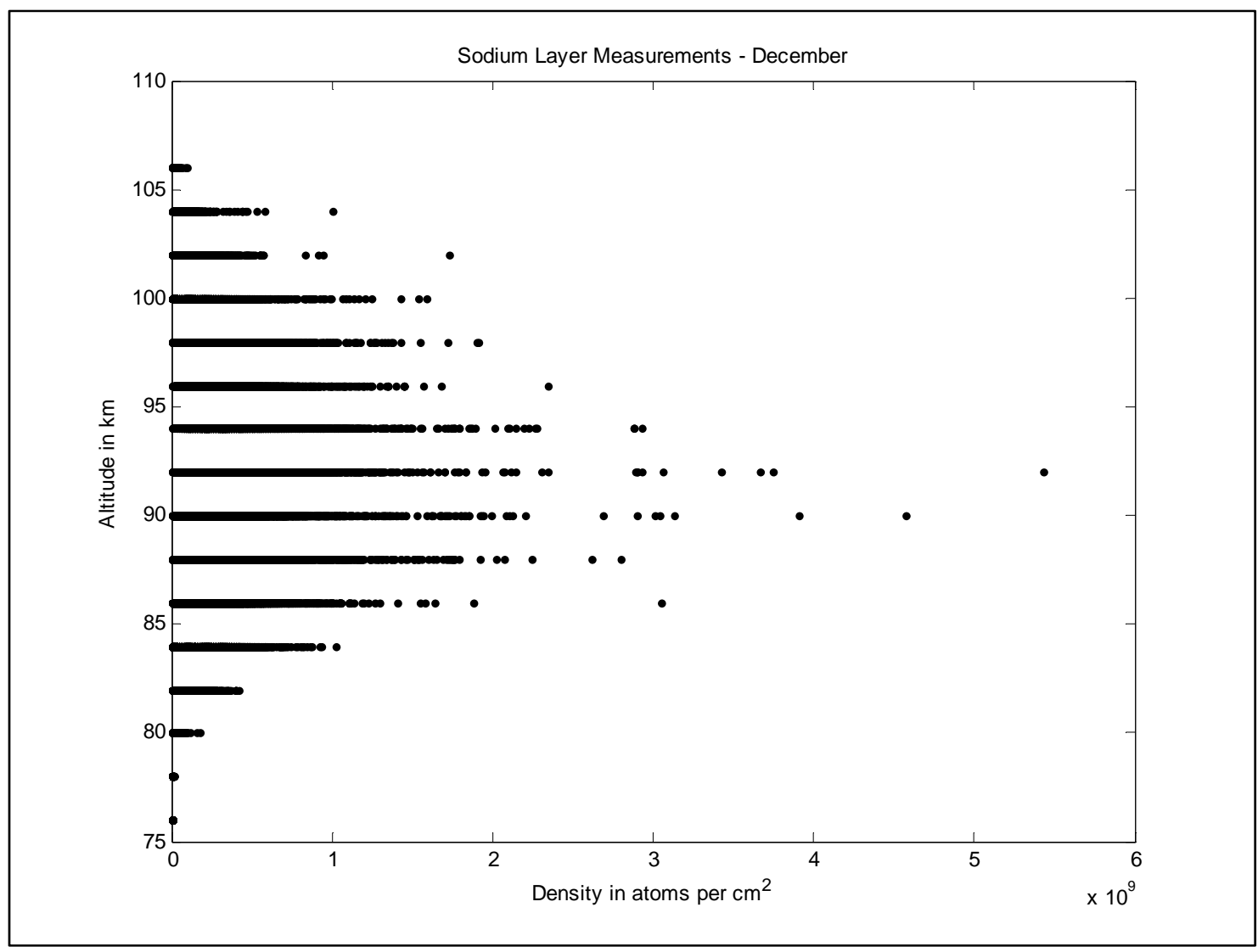

Figure 29: Sodium layer density vs altitude for December for all collected data across the globe 


\section{APPENDIX II}

\section{PLOTS OF GLOBAL CLIMOTOLOGY PRESENTED IN LEEDR}

In appendix II, the global view of the sodium distribution can be seen based off of the collected data from the OSIRIS sensor from 2004 to 2011. There is a global plot for each month to show how the distribution changes globally. Horizontal boundary line can be seen and are representative of where no data was collected past. This is expected due to the process in which the data was collected. Most notably, no data was able to be collected in the northern most latitudes during winter months and southern most latitudes during summer months.

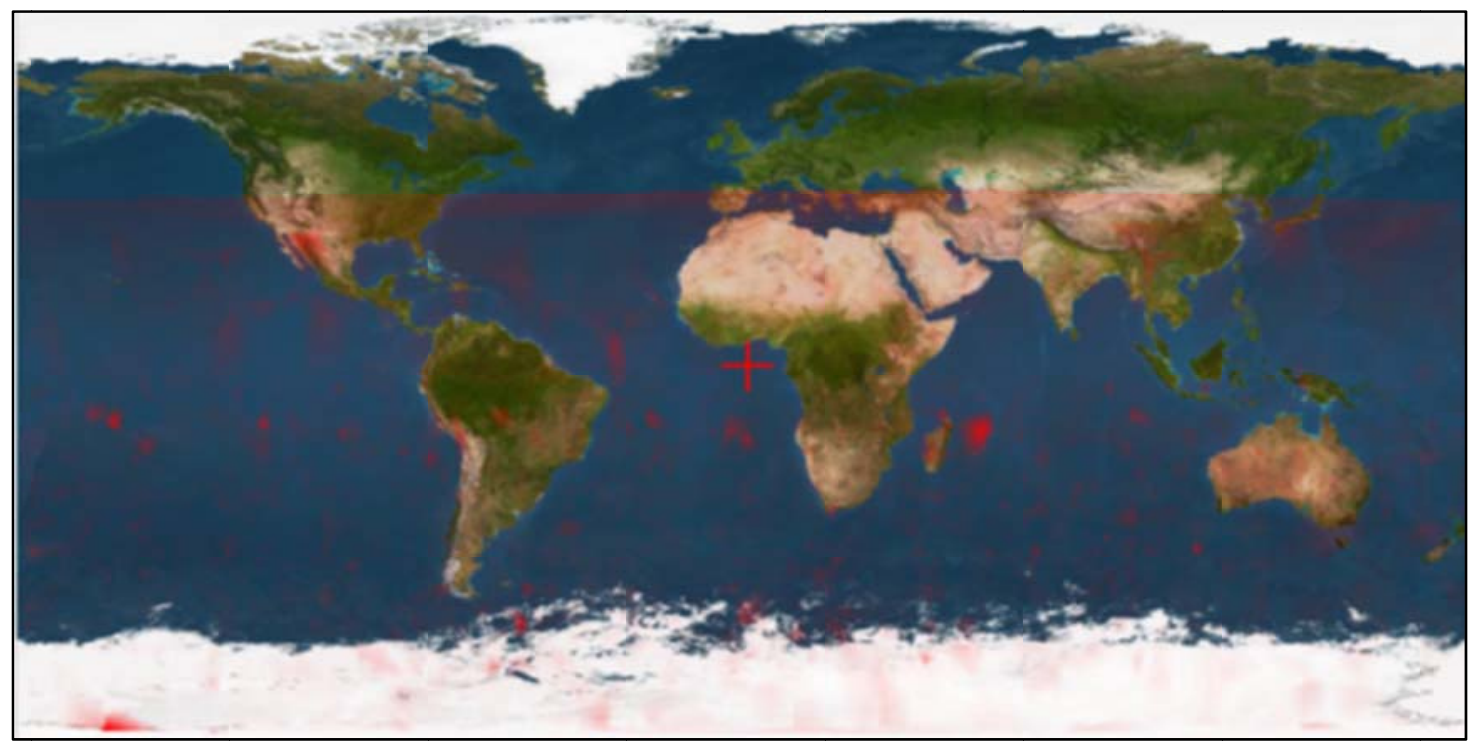

Figure 30: LEEDR plot of global sodium layer density distribution for January 


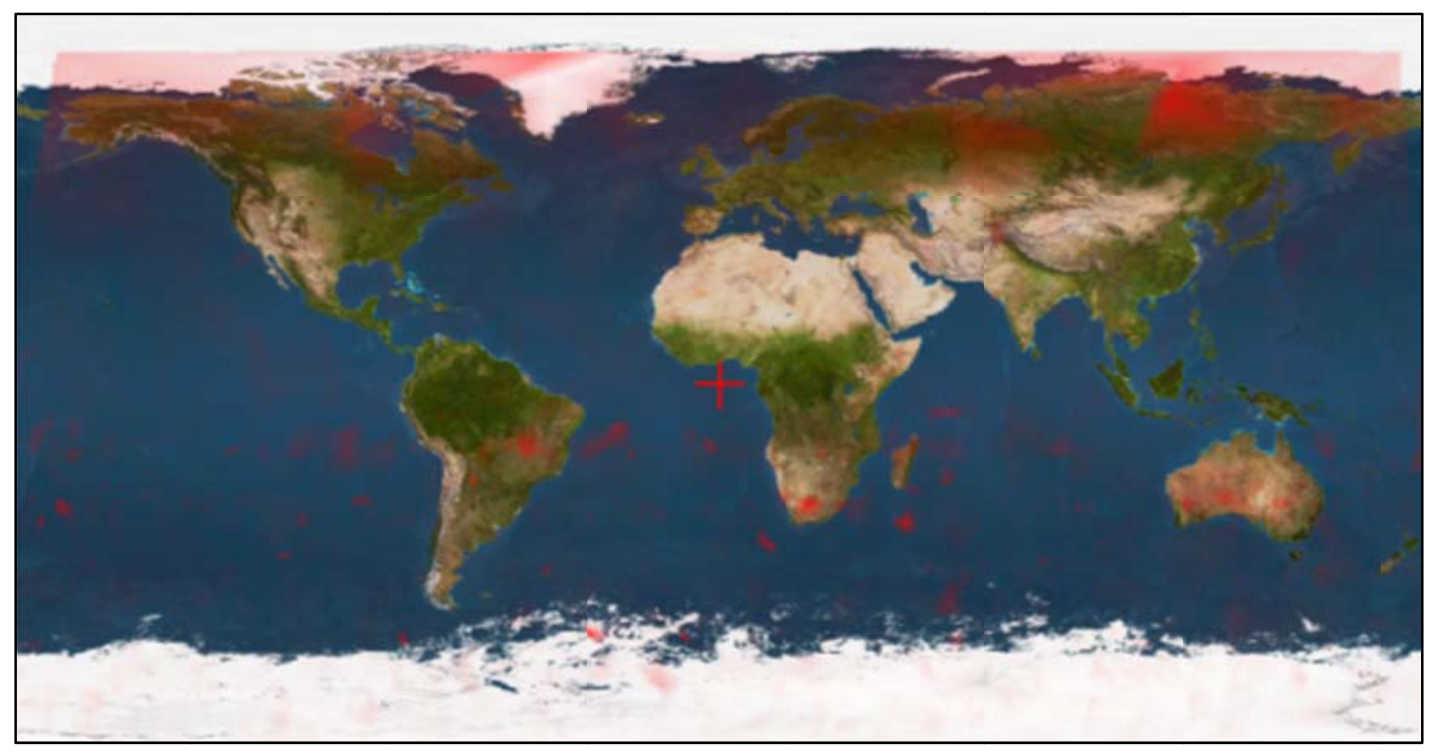

Figure 31: LEEDR plot of global sodium layer density distribution for February

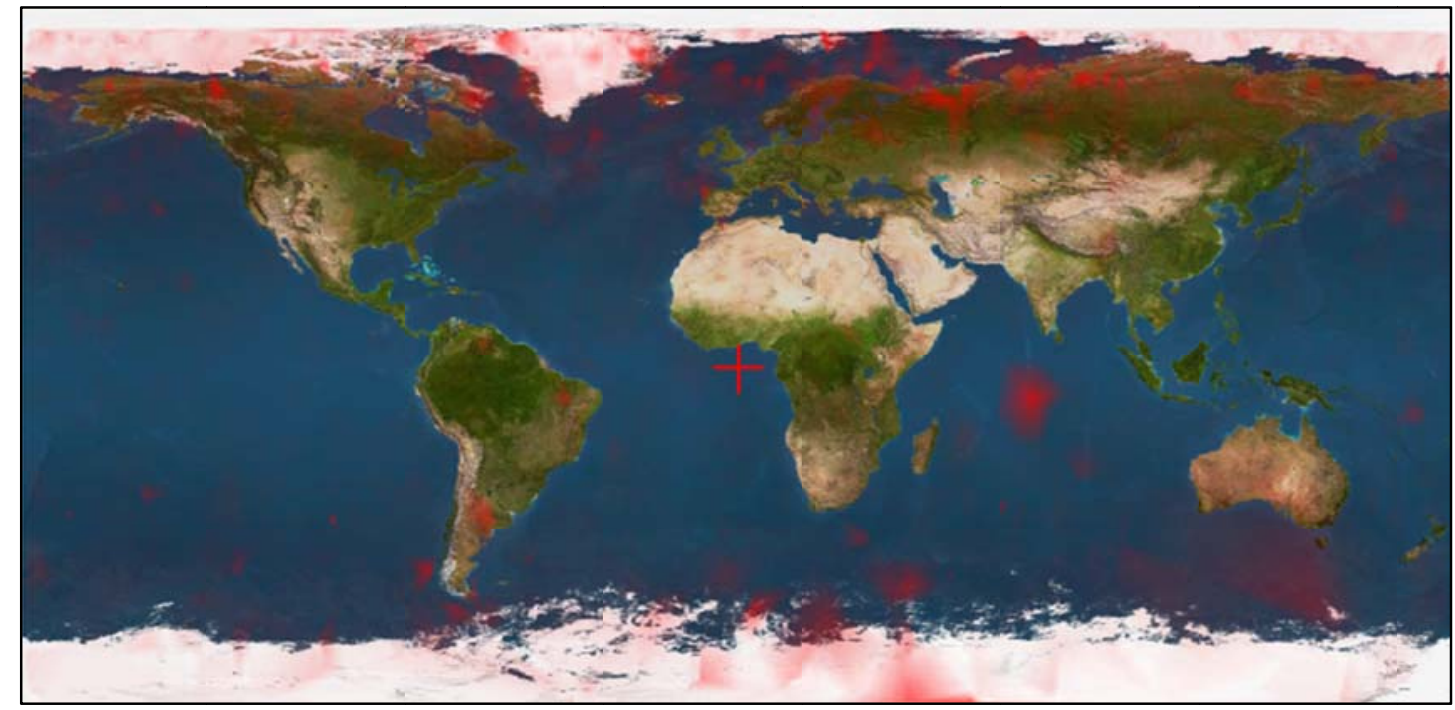

Figure 32: LEEDR plot of global sodium layer density distribution for March 


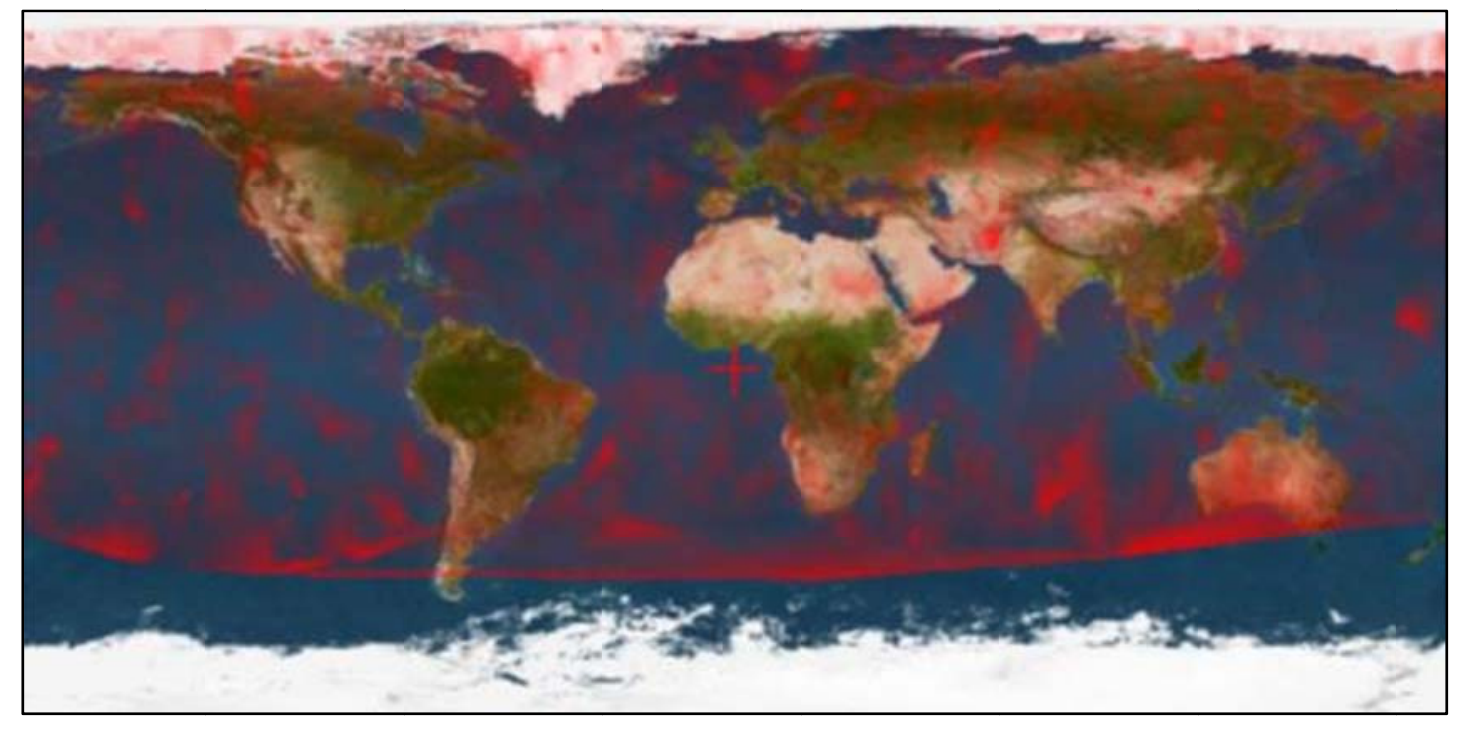

Figure 33: LEEDR plot of global sodium layer density distribution for April

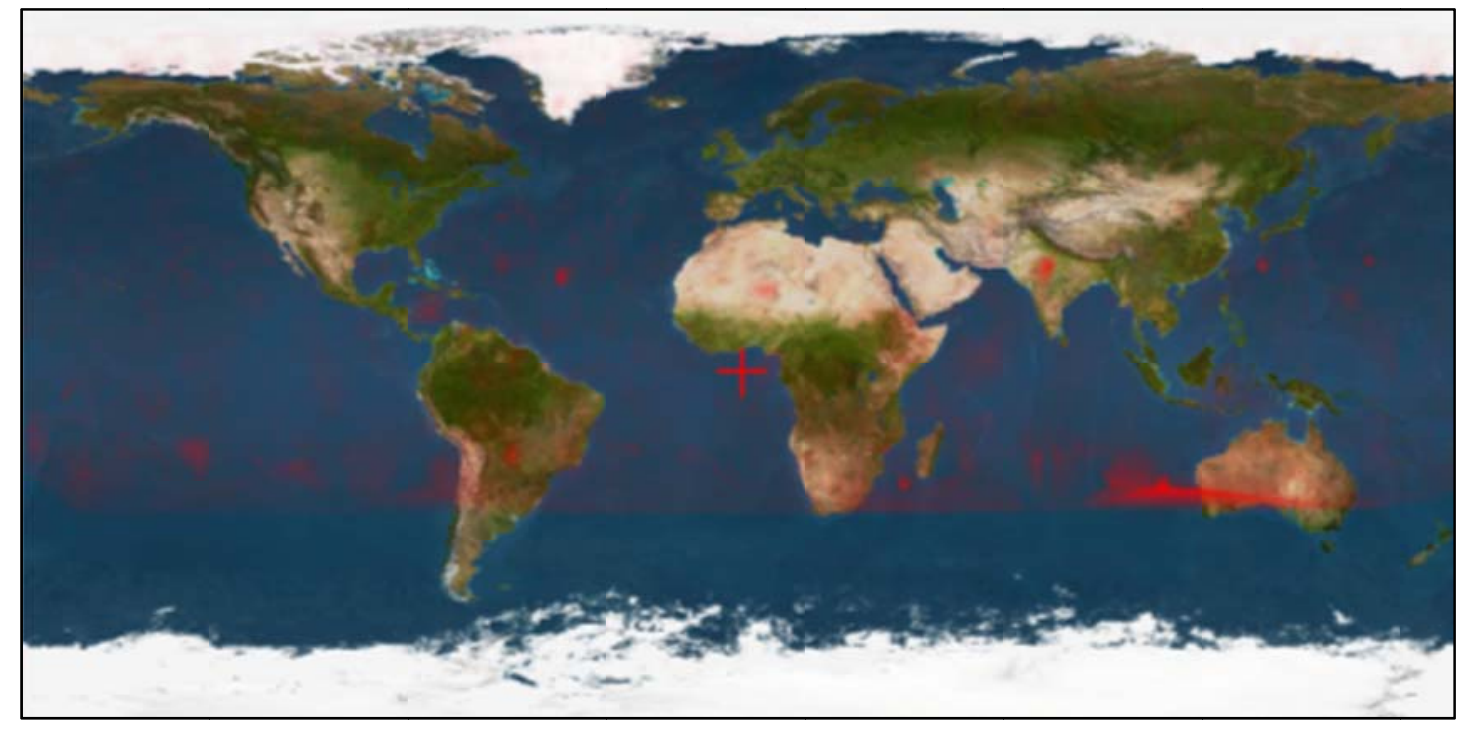

Figure 34: LEEDR plot of global sodium layer density distribution for May 


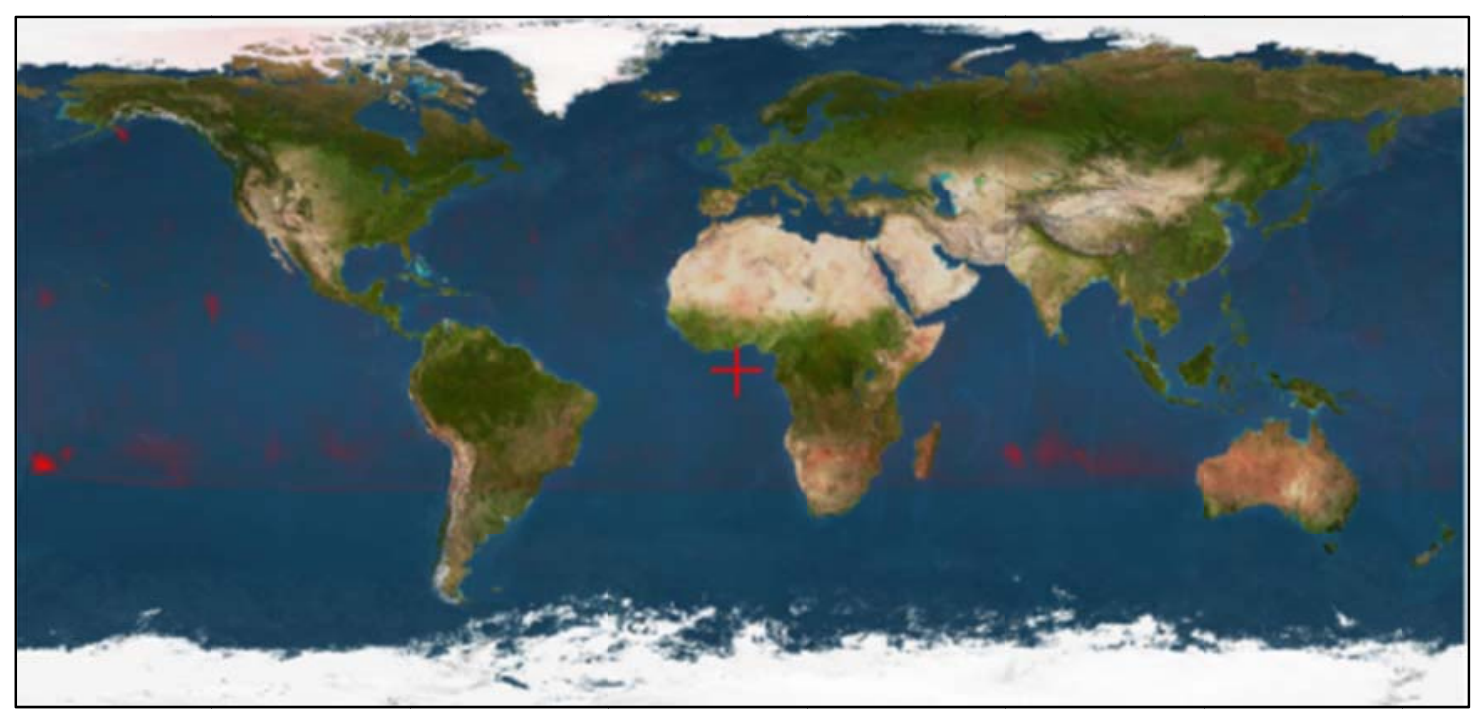

Figure 35: LEEDR plot of global sodium layer density distribution for June

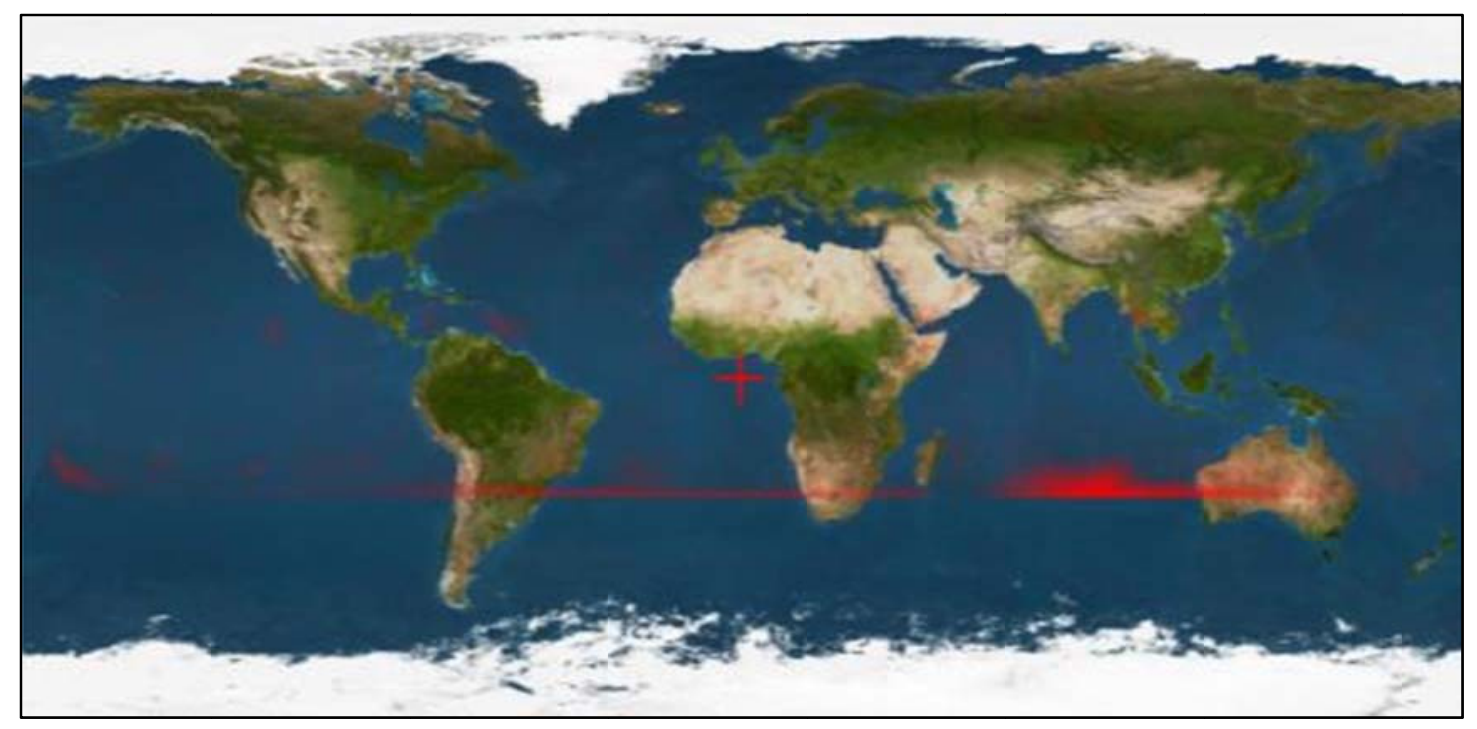

Figure 36: LEEDR plot of global sodium layer density distribution for July 


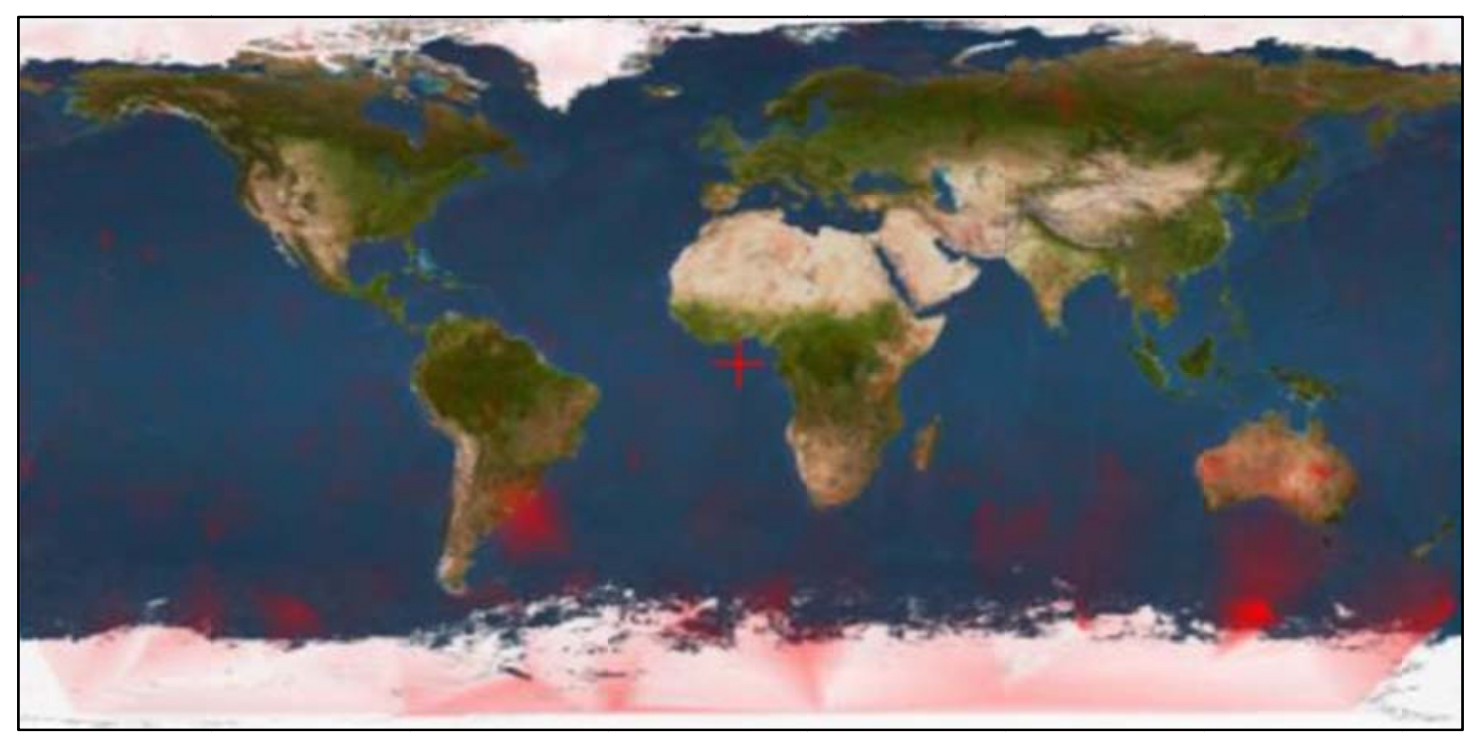

Figure 37: LEEDR plot of global sodium layer density distribution for August

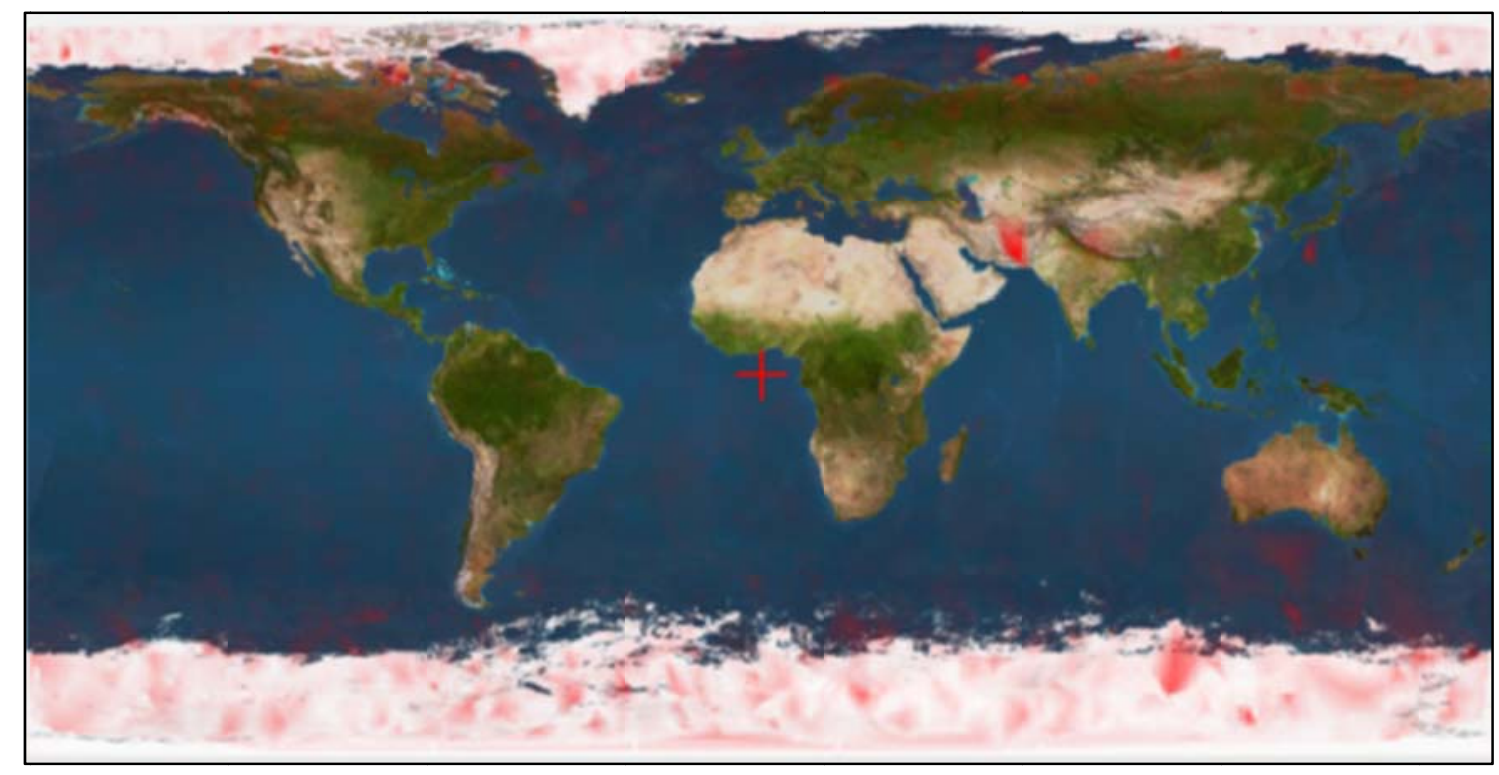

Figure 38: LEEDR plot of global sodium layer density distribution for September 


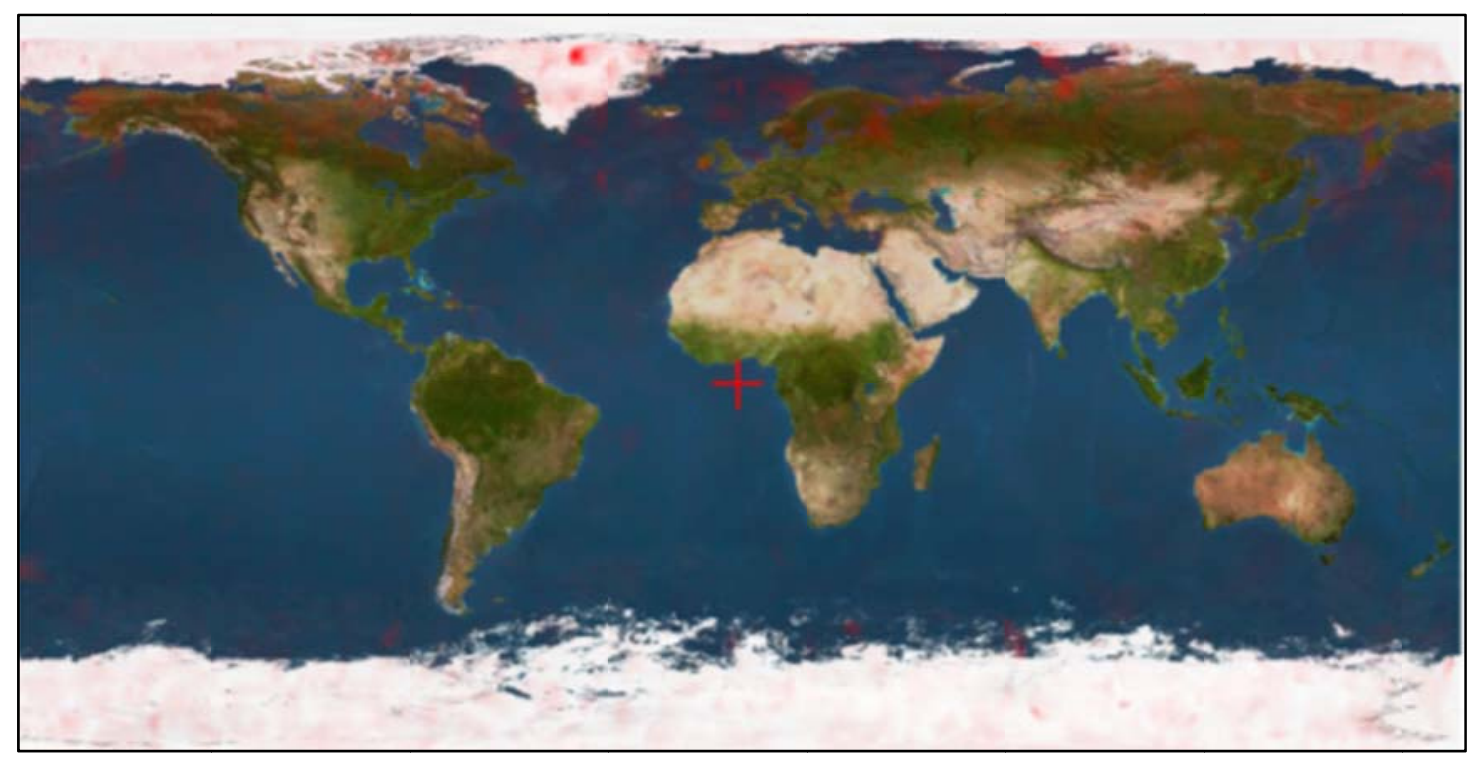

Figure 39: LEEDR plot of global sodium layer density distribution for October

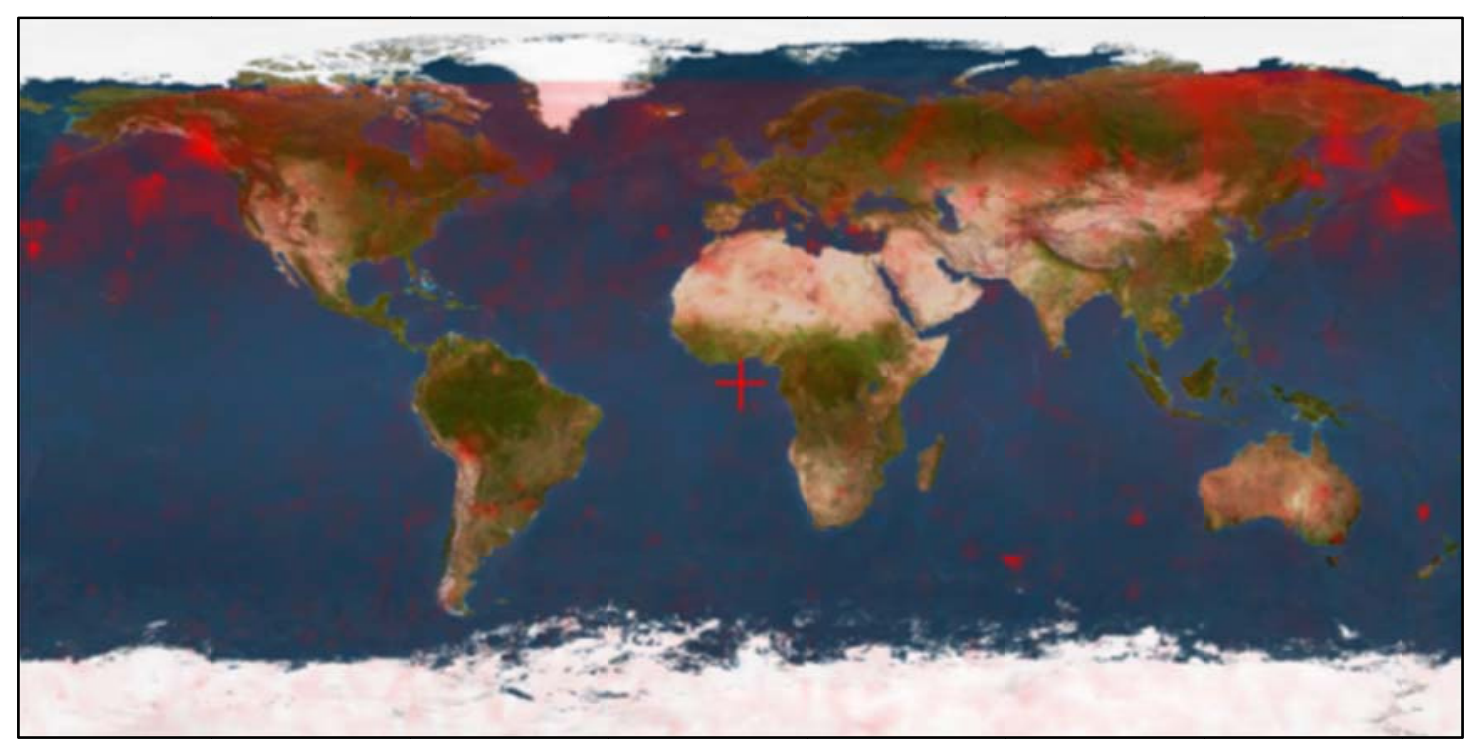

Figure 40: LEEDR plot of global sodium layer density distribution for November 


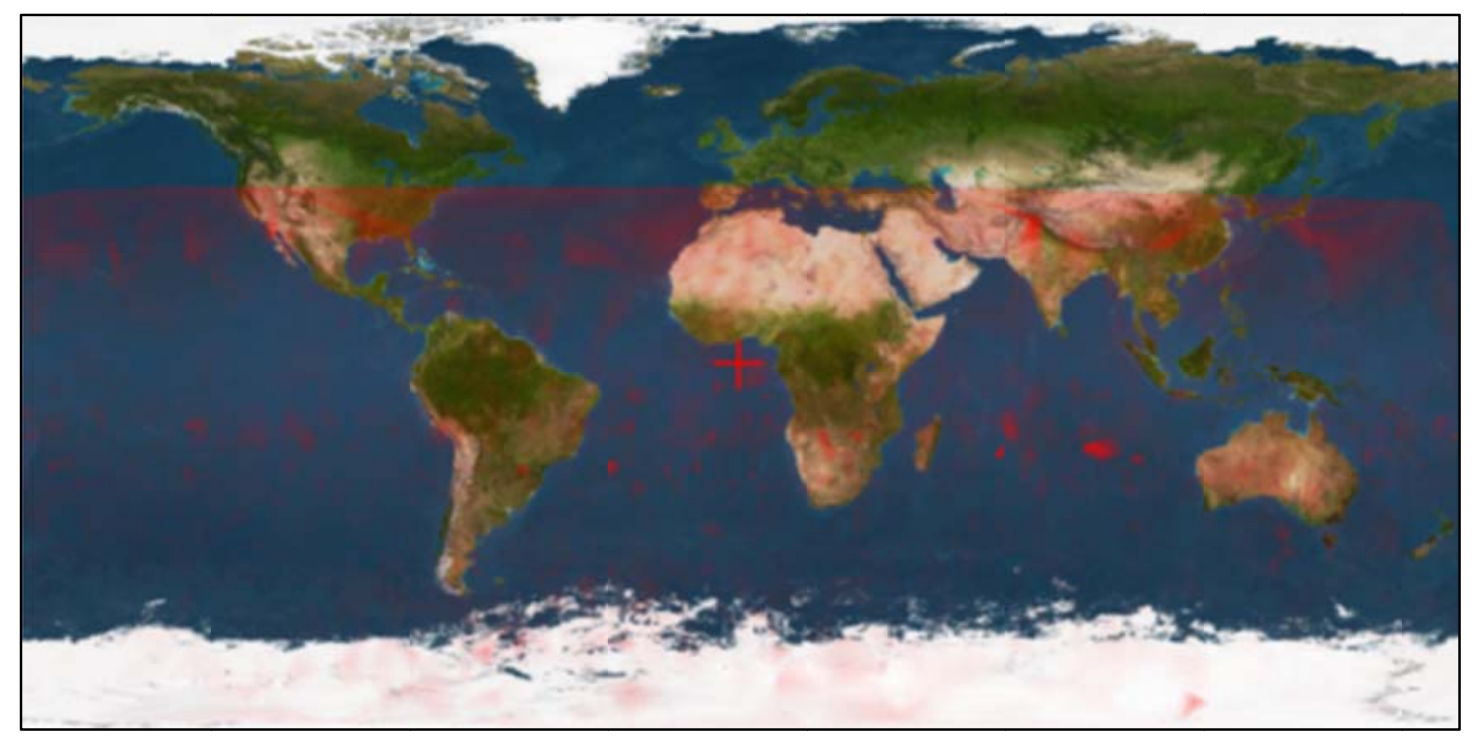

Figure 41: LEEDR plot of global sodium layer density distribution for December 
APPENDIX III

\section{RESULTS FROM GLOBAL SITE SPECIFIC SCENARIOS}

Appendix III shows how specific regions on earth would perform as a sodium guidestar adaptive optic system. Thirty-five locations were chosen to be representative of all major regions in all seven continents. A central point for each location was chosen and data was averaged together within a 10 degree latitude and longitude radius to create the data displayed in each figure. The solid red horizontal line represents the sodium column abundance needed to create a brightness magnitude of 12 sodium guidestar from a typical adaptive optic system, as presented in this paper. Of note, that threshold would be lowered if a more capable adaptive optic system was to be used. 


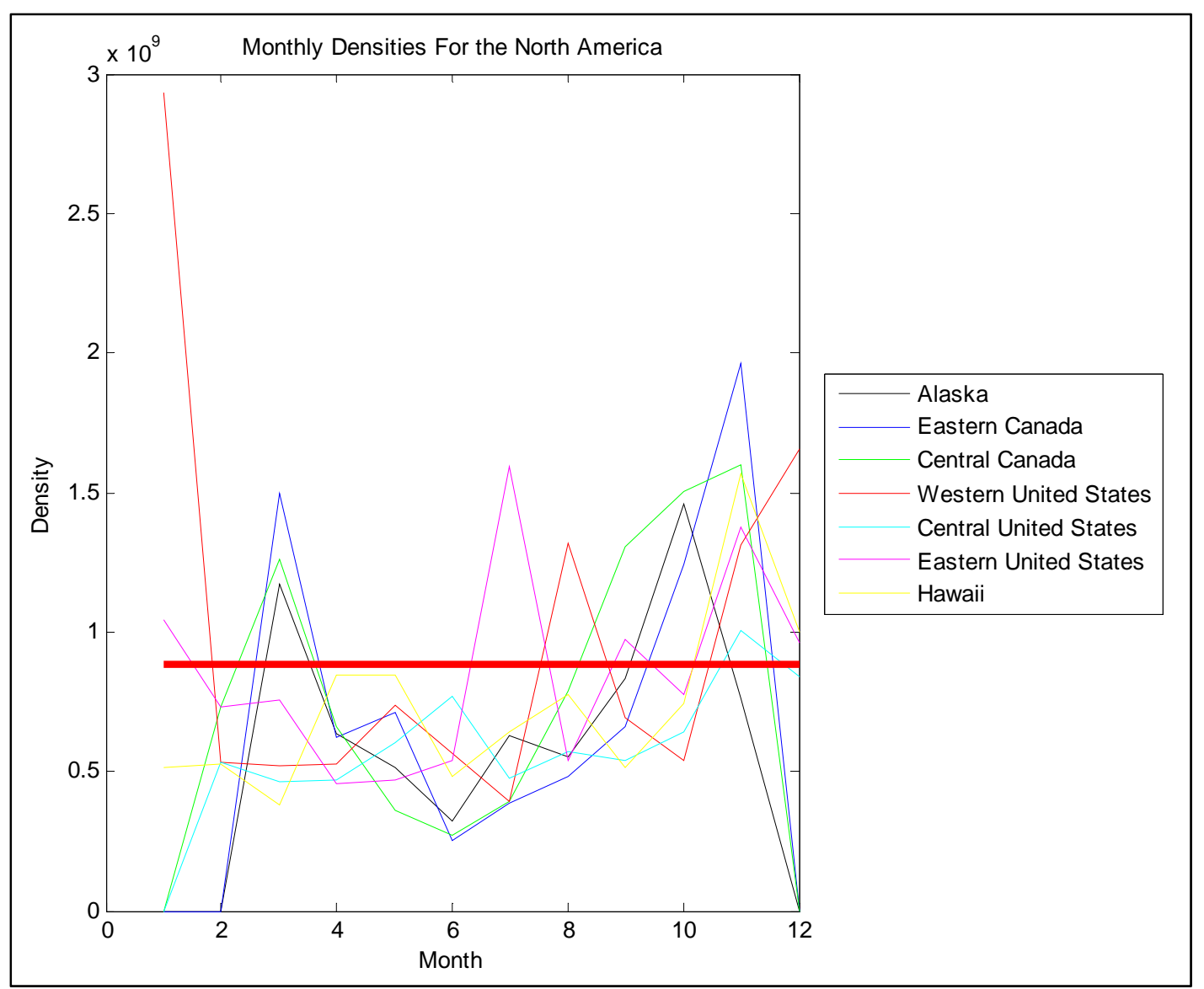

Figure 42: Maximum monthly sodium layer density for location in North America 


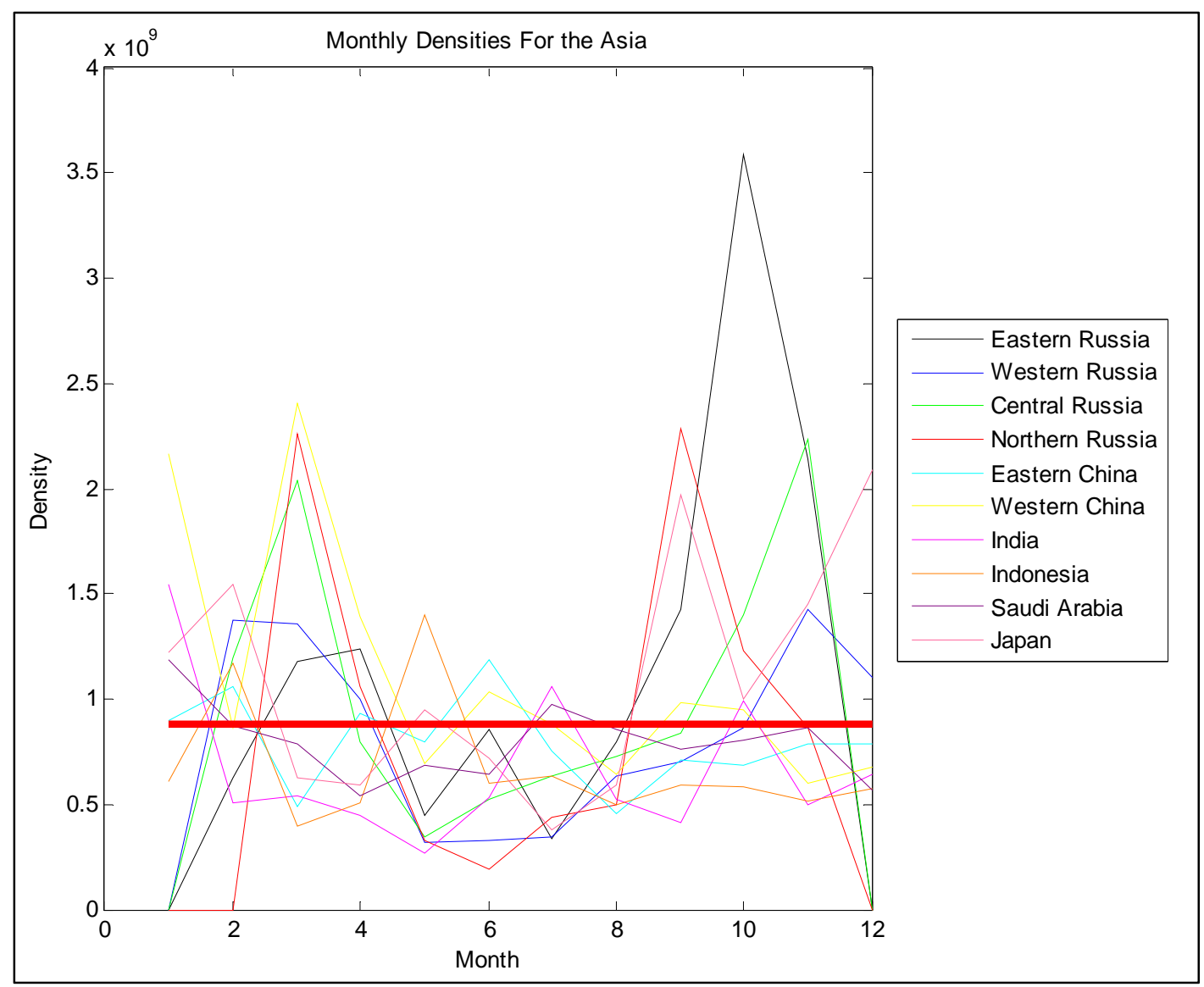

Figure 43: Maximum monthly sodium layer density for location in Asia 


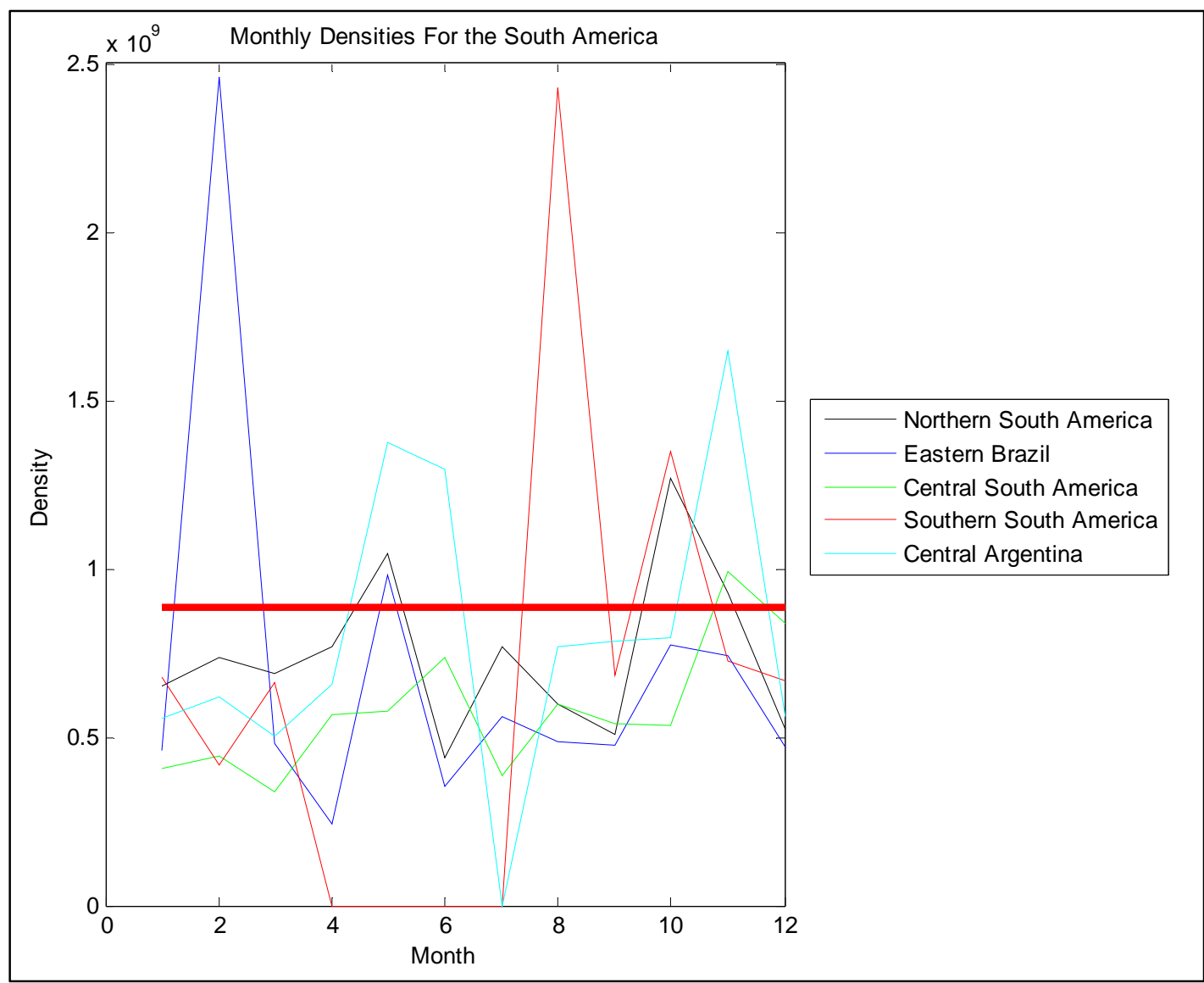

Figure 44: Maximum monthly sodium layer density for location in South America 


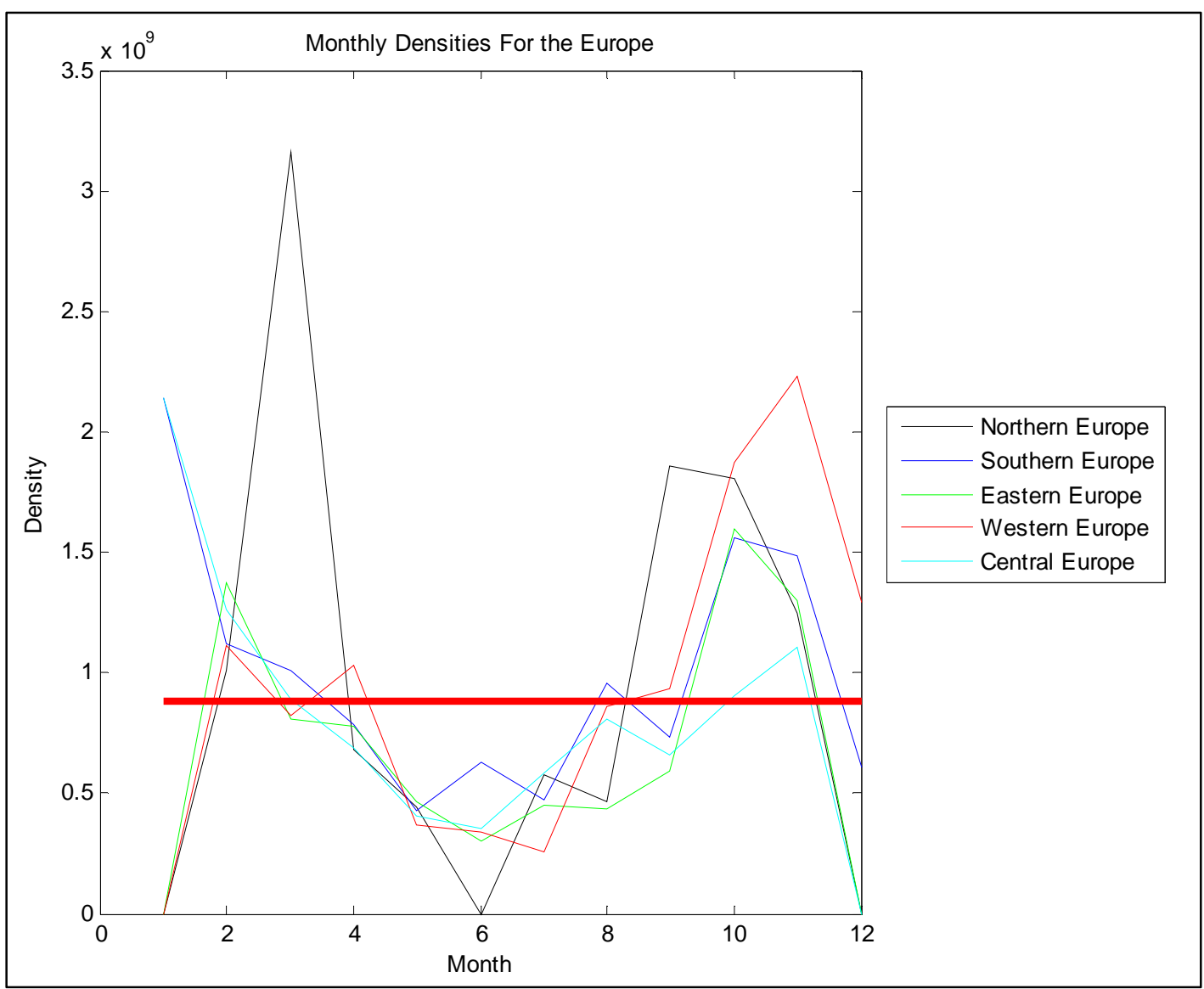

Figure 45: Maximum monthly sodium layer density for location in Europe 


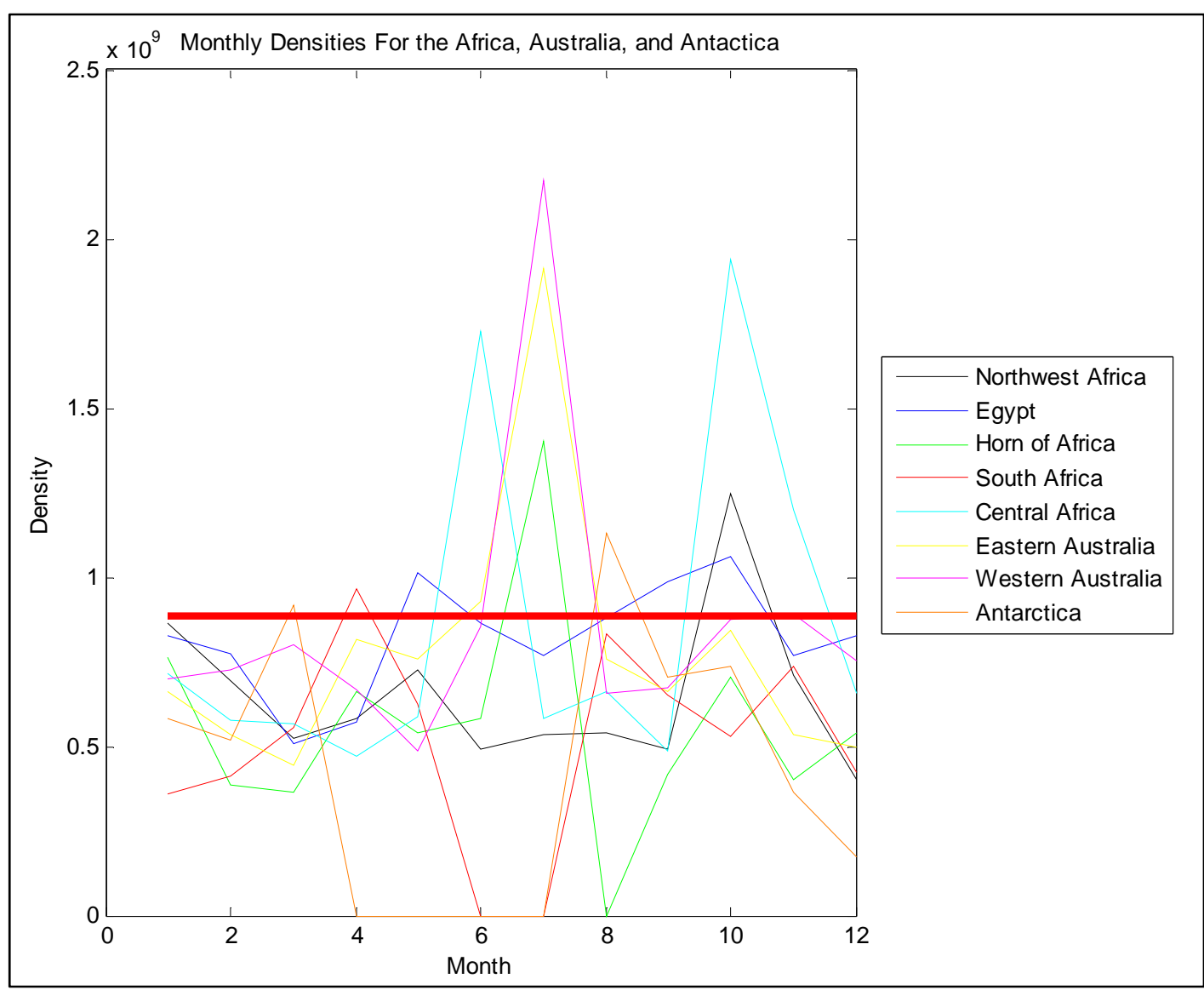

Figure 46: Maximum monthly sodium layer density for location in Africa, Australia, and Antarctica 